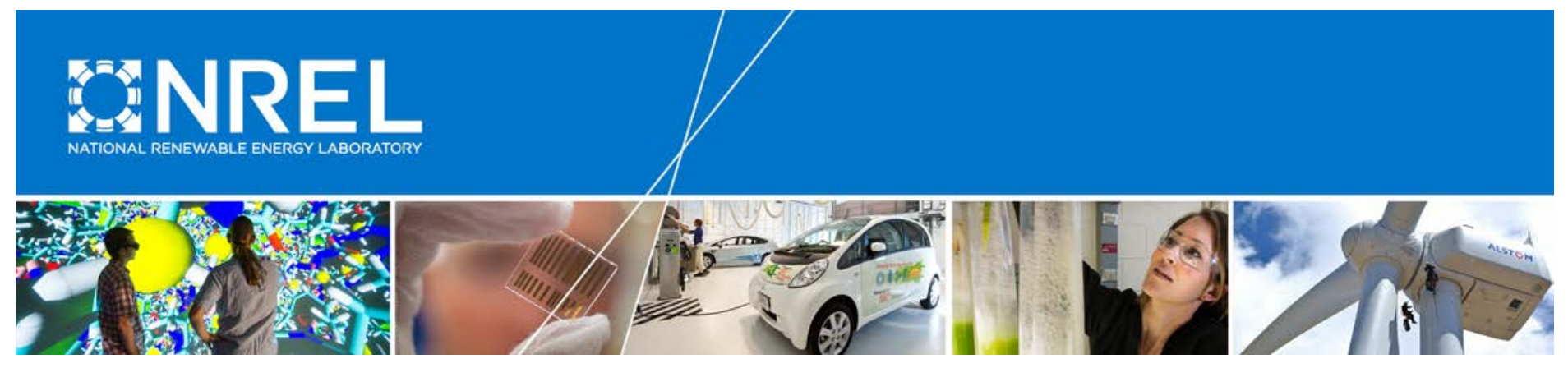

\title{
Vegetation and Wildlife Surveys at the National Renewable Energy Laboratory, South Table Mountain
}

\section{June 2010 - May 2011}

Walsh Environmental Scientists and Engineers, LLC Boulder, Colorado

NREL Technical Monitor: Tom Ryon

NREL is a national laboratory of the U.S. Department of Energy Office of Energy Efficiency \& Renewable Energy Operated by the Alliance for Sustainable Energy, LLC

This report is available at no cost from the National Renewable Energy Laboratory (NREL) at www.nrel.gov/publications.

Subcontract Report NREL/SR-1900-70464 July 2018 


\section{Vegetation and Wildlife Surveys at the National Renewable Energy Laboratory, South Table Mountain}

\section{June 2010 - May 2011}

Walsh Environmental Scientists and

Engineers, LLC

Boulder, Colorado

NREL Technical Monitor: Tom Ryon

Prepared under Subcontract No. LGF-0-40734-01

\section{Suggested Citation}

Walsh Environmental Scientists and Engineers, LLC (2018).

Vegetation and Wildlife Surveys at the National Renewable Energy Laboratory, South Table Mountain: June 2010 - May 2011. Golden, CO: National Renewable Energy Laboratory. NREL/SR-1900-70464.

https://www.nrel.gov/docs/fy18osti/70464.pdf.

NREL is a national laboratory of the U.S. Department of Energy

Office of Energy Efficiency \& Renewable Energy

Operated by the Alliance for Sustainable Energy, LLC

This report is available at no cost from the National Renewable Energy Laboratory (NREL) at www.nrel.gov/publications.

National Renewable Energy Laboratory 15013 Denver West Parkway Golden, CO 80401

303-275-3000 • www.nrel.gov

\section{Subcontract Report}

NREL/SR-1900-70464

July 2018

Contract No. DE-AC36-08G028308 
This publication was reproduced from the best available copy submitted by the subcontractor and received no editorial review at NREL.

\section{NOTICE}

This work was authored by the National Renewable Energy Laboratory, operated by Alliance for Sustainable Energy, LLC, for the U.S. Department of Energy (DOE) under Contract No. DE-AC36-08G028308. Funding provided by the U.S. Department of Energy Office of Energy Efficiency and Renewable Energy. The views expressed in the article do not necessarily represent the views of the DOE or the U.S. Government.

This report is available at no cost from the National Renewable Energy Laboratory (NREL) at www.nrel.gov/publications.

U.S. Department of Energy (DOE) reports produced after 1991 and a growing number of pre-1991 documents are available free via www.OSTI.gov.

Cover Photos by Dennis Schroeder: (left to right) NREL 26173, NREL 18302, NREL 19758, NREL 29642, NREL 19795.

NREL prints on paper that contains recycled content. 


\title{
2010-2011 Vegetation and Wildlife Surveys at the National Renewable Energy Laboratory, South Table Mountain
}

\author{
Jefferson County, Colorado
}

\section{August 12, 2011}

Purchase Order Number 19601000

Technical Monitor: Thomas Ryon

Prepared for

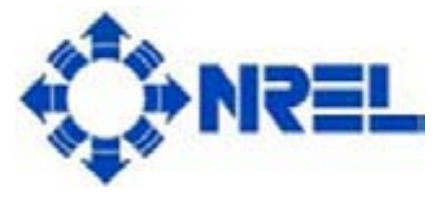

Prepared by

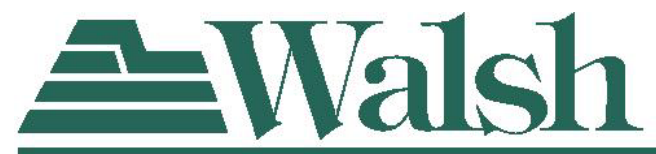

an ecology and environment company 


\section{TABLE OF CONTENTS}

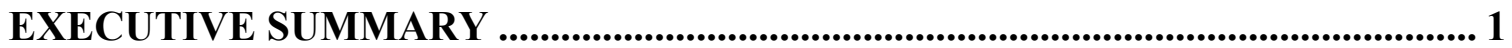

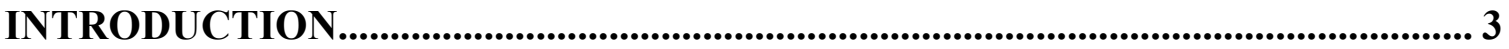

METHODS …….................................................................................................. 3

Vegetation Mapping .............................................................................................. 3

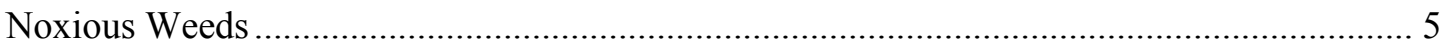

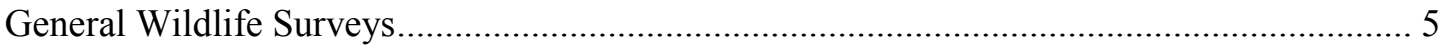

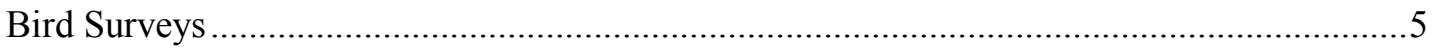

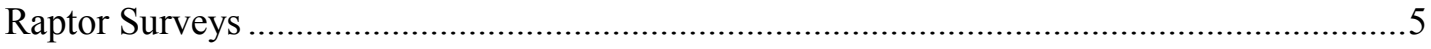

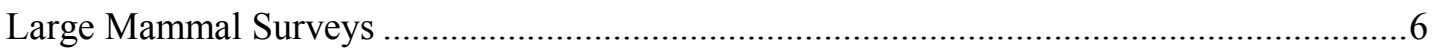

Mammalian Predator Surveys ……………………………………………………….....

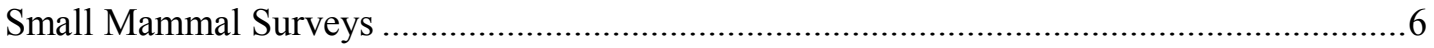

Reptile and Amphibian Survey ....................................................................................

Terrestrial Arthropod Survey …………………………………………………….....

Targeted Wildlife Species......................................................................................... 7

Breeding Bird Density Surveys ......................................................................................

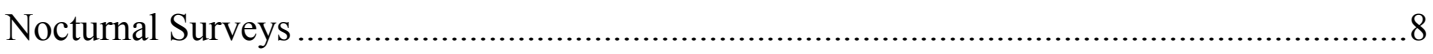

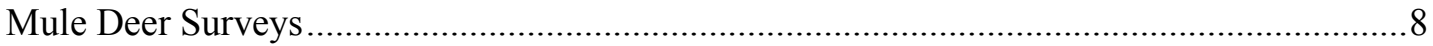

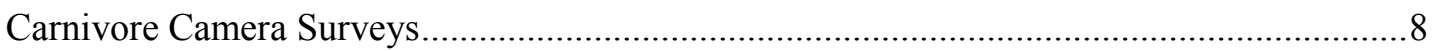

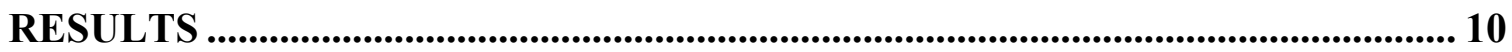

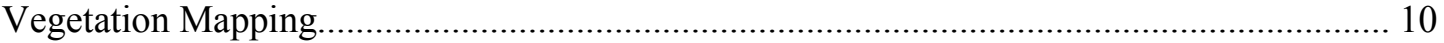

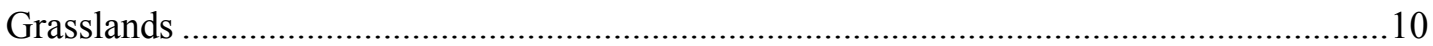

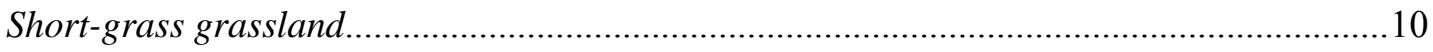

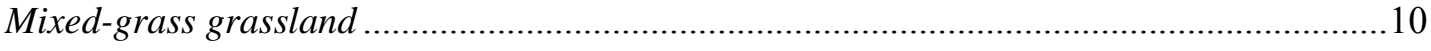

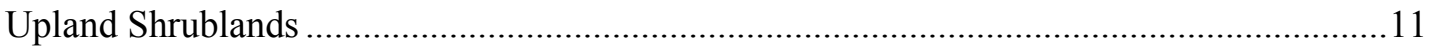

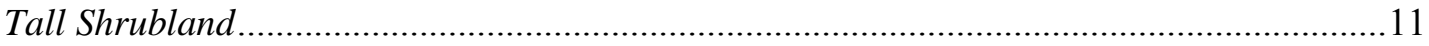

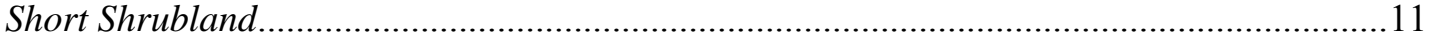

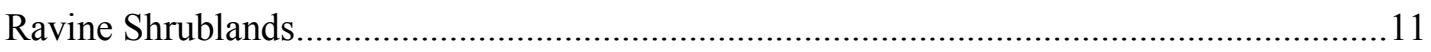

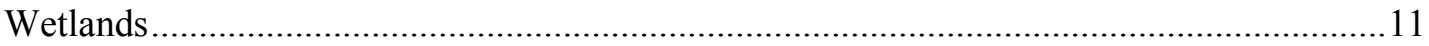

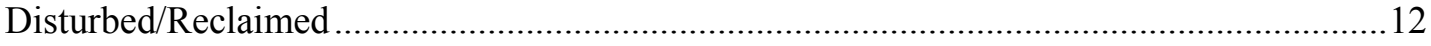

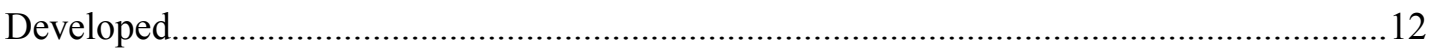

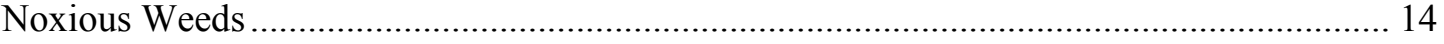

Wildlife Survey Results ………………………………………………………. 16

General Wildlife Surveys................................................................................... 24

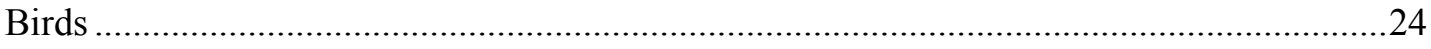

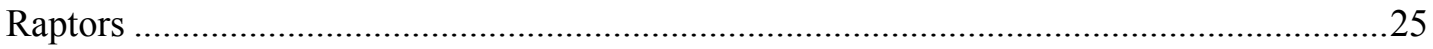

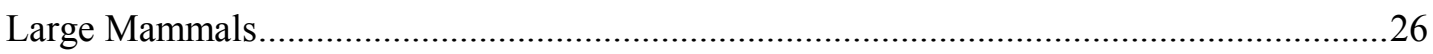

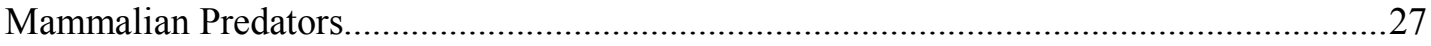

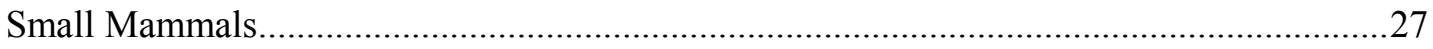

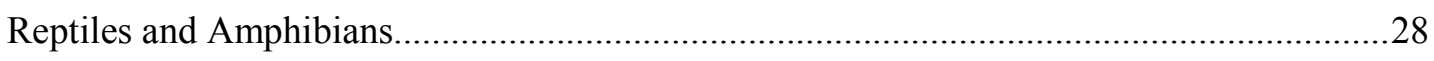




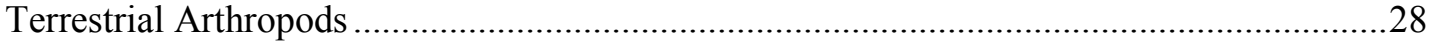

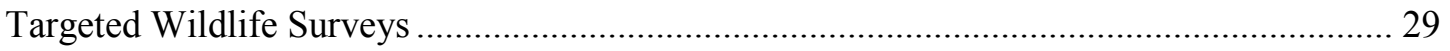

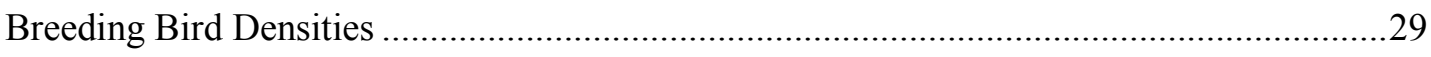

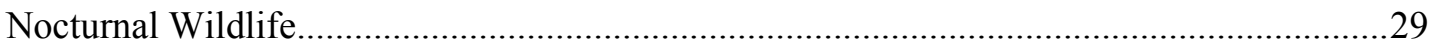

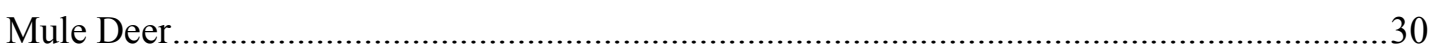

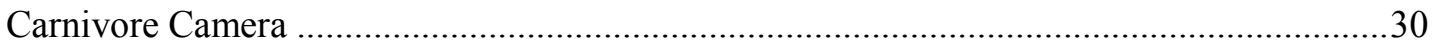

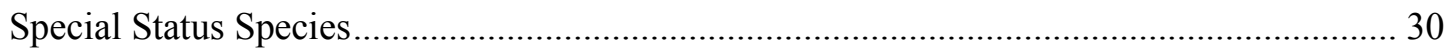

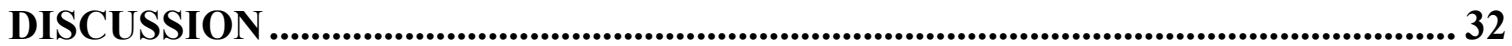

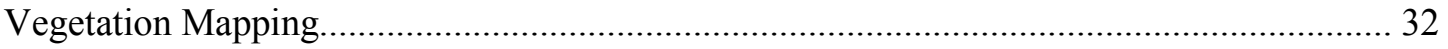

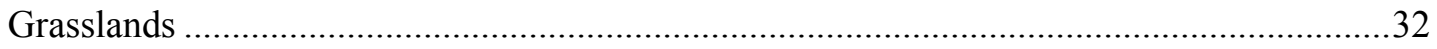

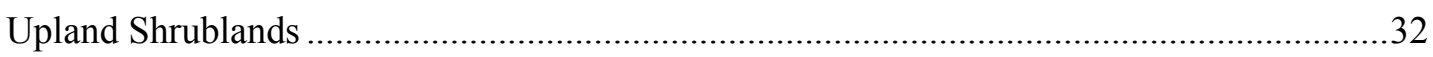

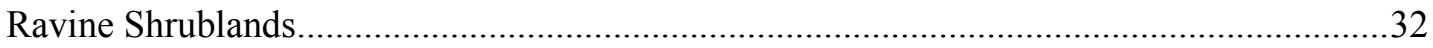

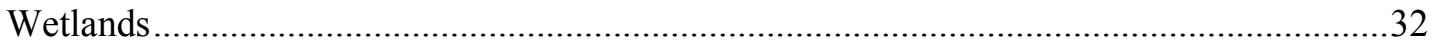

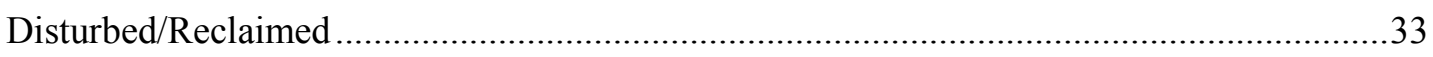

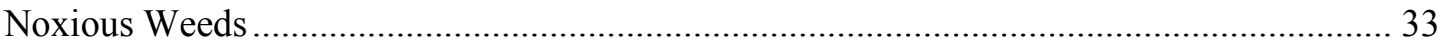

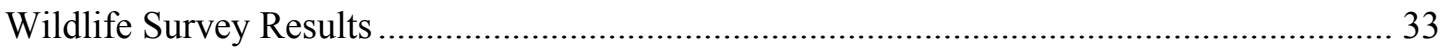

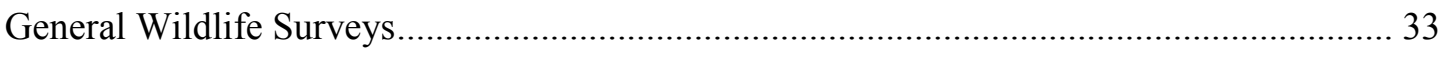

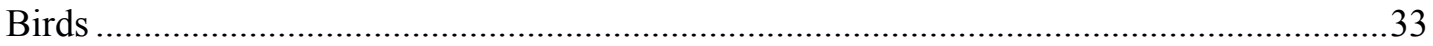

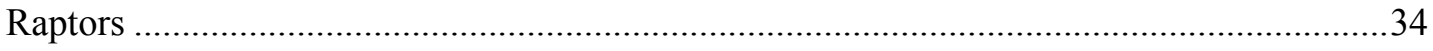

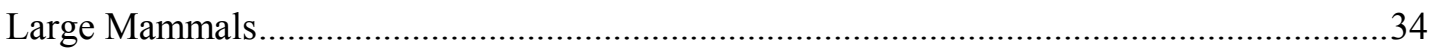

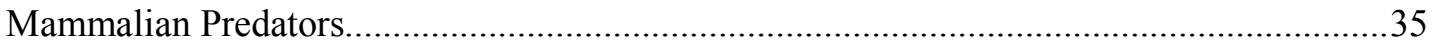

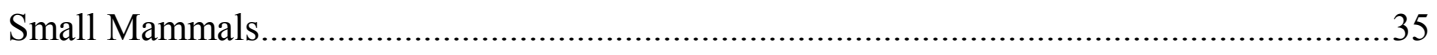

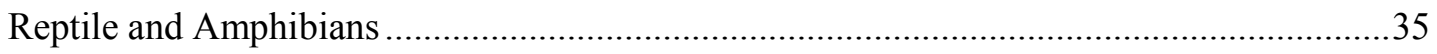

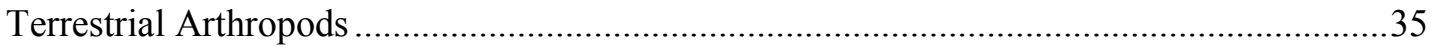

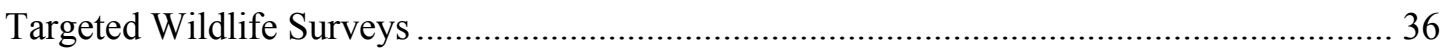

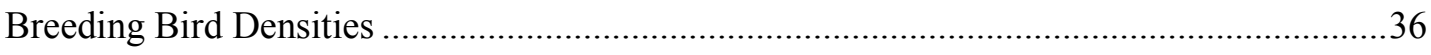

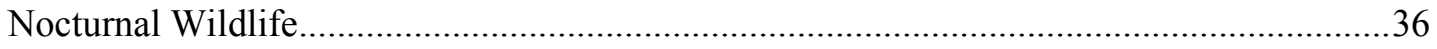

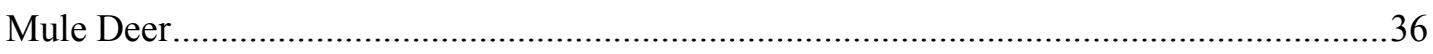

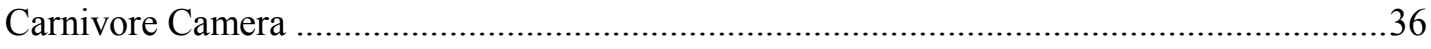

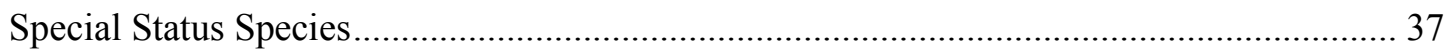

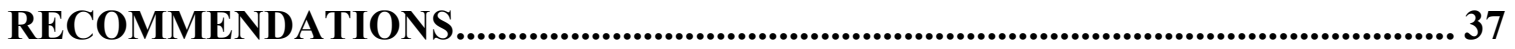

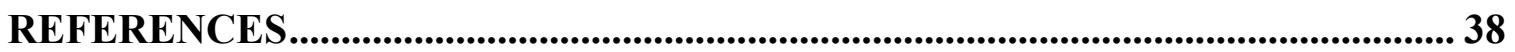

\section{TABLES}

Table 1. Noxious Weed Species Identified at the NREL South Table Mountain Site, June 201014

Table 2. Wildlife Survey Seasons, NREL South Table Mountain Site, 2010-2011 .................... 16

Table 3. Wildlife Survey Results, NREL South Table Mountain Site, 2010-2011 ...................... 17

Table 4. Avian Species Richness, Diversity (Dominance) and Evenness, Point Count Surveys, NREL South Table Mountain Site, 2010-2011 ...................................................................... 24

Table 5. Raptor Survey Results, NREL South Table Mountain Site, 2010 and 2011 ................. 25 
Table 6. Mammalian Predator Survey Results, NREL South Table Mountain Site, 2010 and 2011

Table 7. Small Mammal Captures, NREL South Table Mountain Site, 2010 and 2011 .............. 28

Table 8. Breeding Bird Density Estimates, NREL South Table Mountain Site, 2011 29

\section{FIGURES}

Figure 1. General Wildlife Survey Stations and Transects, NREL South Table Mountain Site, 2010 and 2011

Figure 2. Targeted Wildlife Species Stations and Transects, NREL South Table Mountain Site, 2010 and 2011

Figure 3. Vegetation Map, NREL South Table Mountain Site, 2010 and 2011......................... 13

Figure 4. Weed Map, NREL South Table Mountain Site, 2010 and 2011 ................................. 15

Figure 5. Number of Pellet Groups Observed per Vegetation Type, Large Mammal Surveys,

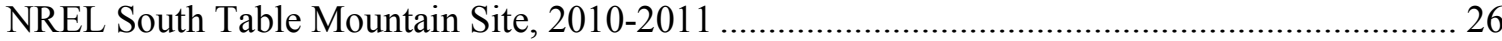

Figure 6. Mule Deer Concentrated Use Observations, NREL South Table Mountain Site, 20102011

\section{APPENDICES}

Appendix A Plant Community Species Lists

Appendix B Photos

Appendix C Colorado Natural Heritage Program Data Query Response 


\section{LIST OF ACRONYMS}

$\begin{array}{ll}\text { AIC } & \text { Akaike's information criterion } \\ \text { CDOA } & \text { Colorado Department of Agriculture } \\ \text { CDOW } & \text { Colorado Division of Wildlife } \\ \text { CNHP } & \text { Colorado Natural Heritage Program } \\ \text { DOE } & \text { U.S. Department of Energy } \\ \text { DRHW } & \text { Dinosaur Ridge Hawk Watch } \\ \text { GIS } & \text { Geographic Information System } \\ \text { GPS } & \text { Global Positioning System } \\ \text { HMANA } & \text { Hawk Migration Association of North America } \\ \text { n } & \text { Total number of detected birds } \\ \text { NEPA } & \text { National Environmental Policy Act } \\ \text { NREL } & \text { National Renewable Energy Laboratory } \\ \text { STM } & \text { South Table Mountain } \\ \text { T \& E } & \text { Threatened and Endangered } \\ \text { USFWS } & \text { U.S. Fish and Wildlife Service } \\ \text { Walsh } & \text { Walsh Environmental Scientists and Engineers, LLC }\end{array}$




\section{EXECUTIVE SUMMARY}

This year-long wildlife and vegetation study was conducted by Walsh Environmental Scientists and Engineers, LLC (Walsh) to update previous vegetation and wildlife baseline reports for the South Table Mountain Site (STM). This report will be used to support future National Environmental Policy Act (NEPA) analyses. STM is located on the southeastern corner of South Table Mountain in Jefferson County and is home to a laboratory center of the National Renewable Energy Laboratory (NREL), a national laboratory of the U.S. Department of Energy (DOE), Office of Energy Efficiency and Renewable Energy, and operated by the Alliance for Sustainable Energy, LLC.

NREL has conducted vegetation and wildlife surveys at STM starting in 1987. Prior to the current study, Plantae (2002) completed a site characterization of vegetation communities and noxious weeds, and FORUM (1987) and Science Applications International Corporation (SAIC 2005) conducted wildlife surveys including migratory birds and raptors.

Background research methods included a review of prior studies conducted at STM and species tracked by the Colorado Natural Heritage Program (CNHP). Field methods included walking transects to map vegetation communities and noxious weeds. Numerous wildlife surveys (bird, raptor, large mammal, mammalian predator, small mammal, reptile and amphibian, and terrestrial arthropod) were conducted. Several targeted wildlife surveys were conducted for: breeding bird density, nocturnal wildlife (playback for small owls, amphibian call, and bat acoustical), mule deer, and carnivores (motion-sensing cameras). Special Status Species - those listed as Threatened, Endangered, Candidate, or Species of Special Concern by the U.S. Fish and Wildlife Service (USFWS) or the Colorado Division of Wildlife (CDOW) - were also conducted.

Results include the following:

- The majority of vegetation at STM belongs to the grassland community type. Within that association, there are two distinct community types: short-grass grassland on the mesa top and mixed-grass grassland located on the slopes and toe area. Other mapped vegetation communities at STM include ravine shrubland, tall shrubland, short shrubland, and wetlands.

- Changes in observed vegetation patterns and trends since the 2002 Plantae report include a general increase in noxious weed species diversity and cover throughout the various plant communities.

- Small mammal trapping resulted in a low species richness with only three species captured onsite over two survey periods, Mexican woodrat (Neotoma mexicana), deer mouse (Peromyscus maniculatus), and western harvest mouse (Reithrodontomys megalotis).

- Mammalian predator surveys detected red fox (Vulpes vulpes), coyote (Canis latrans), and striped (Mephitis mephitis) and spotted skunk (Spilogale gracilis). Only coyotes had been detected previously.

- Amphibian call surveys detected one species, Woodhouse's toad (Bufo woodhousii), on the northwestern boundary of STM. Playback surveys failed to detect any owls. Motiondetection cameras detected several coyotes. No bat species were detected.

- General wildlife surveys detected common genera and species expected for the habitats and region of STM.

- The seasonal average for wildlife species richness, diversity (dominance) and total number of detections all approximately doubled since reported for 2004/2005. 
- One-hundred and two (102) wildlife species were detected: five herptiles, 86 birds, and 11 mammals. In comparison FORUM observed a total of 31 species in 1987, and SAIC observed 69 species in 2004/2005.

- One Special Status Species was observed, the peregrine falcon (Falco pereginus), a State Species of Special Concern. 


\section{INTRODUCTION}

This year-long wildlife and vegetation study was conducted by Walsh Environmental Scientists and Engineers, LLC (Walsh) to update previous vegetation and wildlife baseline reports for the South Table Mountain Site (STM). This baseline report will be used to support future National Environmental Policy Act (NEPA) analyses. STM is located on the southeastern corner of South Table Mountain in Jefferson County and is home to a laboratory center of the National Renewable Energy Laboratory (NREL), a national laboratory of the U.S. Department of Energy (DOE), Office of Energy Efficiency and Renewable Energy, and operated by the Alliance for Sustainable Energy, LLC.

STM is situated on approximately 327 acres north of I-70 and west of the I-70 and Denver West Boulevard interchange. The legal description of the current boundary is: T3S, R70W, Sections 36 and T4S, R70W, Section 1 (Figure 1). The STM site includes laboratory and office space, a visitor's center, and 177 acres protected by a conservation easement. NREL has conducted vegetation and wildlife surveys at STM starting in 1987. Prior to the current study, Plantae (2002) completed a site characterization of vegetation communities and noxious weeds, and FORUM (1987) and Science Applications International Corporation (SAIC 2005) conducted wildlife surveys including migratory birds and raptors.

For the current effort, Walsh conducted four seasons of vegetation and wildlife surveys beginning in June, 2010 and ending in May, 2011. This report presents the results of these surveys.

\section{METHODS}

For all studies Walsh and NREL agreed to define the seasons as follows: summer (May, June and July), fall (August, September, and November), winter (December, January, and February), and spring (March, April and May). This was to provide continuity with other studies (SAIC 2005) and NREL's National Wind Technology Center in Jefferson County, Colorado. For this effort, Special Status Species are defined as those listed under the Endangered Species Act (ESA) as Threatened, Endangered (T\&E), or Candidate Species; State T \& E Species; and State Species of Special Concern.

\section{Vegetation Mapping}

Vegetation mapping and characterization were performed following methods described in Plantae (2002). Major plant communities were the basis for both efforts. For the current study, the previously characterized location and area of all major plant communities on the site were checked against current rectified aerial photography and ground-truthed in the field on June 22 and 23, 2010; and May 13, 2011. During the ground-truthing effort, transects were walked at variable intervals through each previously-mapped community. Parallel transects were walked through different portions of the more extensive short-grass grassland and the mixed-grass grassland areas. General conditions for each community type were recorded and comprehensive plant species lists for each community were updated, as necessary (Appendix A). Observations of species dominance as well as on-the-ground discrepancies found between the previous mapping effort and current conditions were noted. Representative photographs of all plant communities were collected (Appendix B). Plant taxonomy follows Weber and Wittmann (2001). 


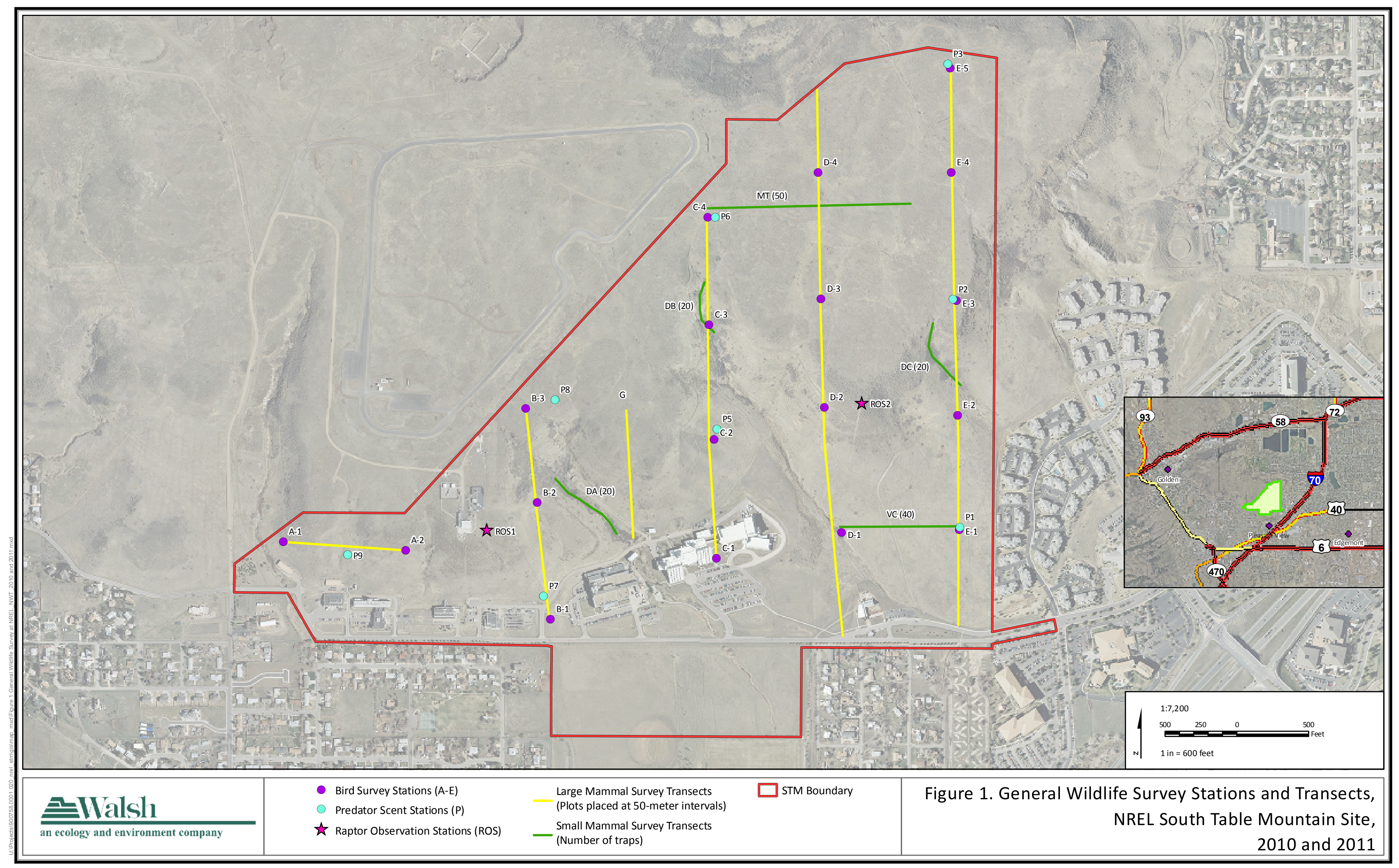

This report is available at no cost from the National Renewable Energy Laboratory (NREL) at www.nrel.gov/publications 


\section{Noxious Weeds}

The location of weeds listed by the Colorado Noxious Weed List (Colorado Division of Agriculture [CDOA] 2010) was assessed on June 22 and 23, 2010 by walking the same transects used for the vegetation mapping. Populations of weeds that occurred in a diffuse pattern with individual or small numbers of plants scattered throughout the landscape were noted but not mapped as distinct polygons. Weed populations with a high density of plants per square meter were mapped as such. A threshold density of approximately 3 to 5 plants per $25 \mathrm{ft}^{2}$ was used to determine whether an area was mapped. Digital location data were collected using Global Positioning System (GPS) receiver units and downloaded into a Geographic Information System (GIS) database. These data were used to estimate cover of weed populations.

\section{General Wildlife Surveys}

Wildlife surveys followed, to the extent practicable, the methods and locations used for the previous survey (SAIC 2005). Note that point count bird surveys are conducted under this section (Bird Surveys), whereas breeding bird density surveys are covered under Targeted Wildlife Surveys (Breeding Bird Density Surveys) and that they use different protocols and produce different types of results.

\section{Bird Surveys}

Bird surveys were conducted at 18 survey points, at 250-meter intervals along transects previously established by SAIC (2005) (Figure 1). Points F-1 and F-2 from the SAIC (2005) study were no longer available due to new developments. During a five-minute sampling period at each point, all observed birds, as well as sex, behavior, distance from the center of the point, and habitat type were recorded. Birds detected between points were recorded to capture any species missed during point counts including any Special Status Species. Walsh conducted two surveys per season, on consecutive mornings. Walsh followed the analysis used by SAIC (2005) to estimate diversity. SAIC (2005) used a metric that is correctly termed dominance and is the reciprocal of the Simpson diversity index (Begon et al. 2006; Van Dyke 2008, p. 91). Evenness was also estimated using species richness and diversity (dominance).

\section{$\underline{\text { Raptor Surveys }}$}

During April and September, the regional peaks for raptor migration, all observed raptors were recorded at two points previously established by SAIC (2005) (Figure 1). Raptor surveys were conducted during mid-day survey periods (10 AM to $2 \mathrm{PM}$ ), which correspond to the daily peak times of raptor migration flights. Each point was surveyed on two consecutive days using the standard protocol established by the Hawk Migration Association of North America (HMANA 2010). This protocol was also used for counting raptors at the Dinosaur Ridge Hawk Watch (DRHW) four miles to the southwest of STM. Species, flight direction, and flight height were recorded. Weather conditions were recorded hourly. Efforts were made to distinguish local nonmigratory raptors from migratory raptors. Calculated variables included number of raptors per hour and per survey.

A site-wide raptor nest survey was conducted in April, each day after migratory raptor surveys were completed. Any raptor nests previously recorded in 2004 were revisited to determine activity status, without causing disturbance to the birds. Additionally, all other appropriate habitats were assessed for nesting activity (trees, buildings, and topographic features). 


\section{Large Mammal Surveys}

Large mammal surveys were based on pellet-group plots, following Neff (1968) and SAIC (2005). Seventy-six circular plots with a four-meter diameter were spaced approximately 50 meters apart along Transects A, B, C, D, E and G established by SAIC (Figure 1). Wooden stakes marked the center of each plot. Pellets were removed from each plot in February 2011. The pellet-group plots were checked in May 2011, and any new pellets were counted, recorded, and removed. The plots are scheduled to be re-checked and recorded again in November 2011 by NREL staff. Mule deer use and relative abundance surveys were also conducted under the targeted wildlife species surveys.

\section{Mammalian Predator Surveys}

Walsh conducted scent-station surveys for predators using the previous methods developed by Linhart and Knowlton (1975) and used by SAIC (2005). Scent stations consisted of a one-meter circle of sifted dirt with a fatty-acid scented predator survey disk (F.A.S. Scented Disk from Pocatello Supply Depot, Pocatello, ID) placed in the center. Nine stations were established, located along the same transects used in the migratory bird surveys, at 300-meter intervals (Figure 1). The scent stations were evenly divided between the mesa top, which consists of shortgrass grassland and tall shrubland communities and the mesa toe, which consists of the mixedgrass grassland community. Stations were checked for tracks of mammalian predators. Two surveys, each lasting three consecutive nights, were conducted during each season. Survey months in 2010 included June, July, September, October, and December. Surveys in 2011 were conducted in January, February, and April. Carnivore camera surveys were also conducted as part of the targeted wildlife species surveys.

\section{$\underline{\text { Small Mammal Surveys }}$}

Small mammal live-trapping was conducted along five transects in three vegetation community types: one in short-grass grassland, three in ravine shrubland, and one in mixed-grass grassland (Figure 1). Two survey sessions were conducted: July 12 to 15, 2010, and April 25 to 28, 2011. Each survey took place over three consecutive nights. One hundred and fifty (150) Sherman live traps were placed five-meters apart along the five transects, resulting in a total of 450 trap nights for each survey session, 900 trap nights for the year.

The protocol employed standard field procedures for small mammal trapping and followed the guidelines approved by the Animal Care and Use Committee of the American Society of Mammalogists (Sikes et al. 2011). Traps were baited with sweet horse feed, and a ball of polyester batting was placed inside each trap for insulation and bedding to prevent hypothermia in captured animals.

The traps were inspected each morning, the species, sex, and age of each trapped animal was determined and recorded. The animals were released at the trap station, and the traps were closed, and then reopened late in the day. Traps were washed at the end of the trapping session in a 10 percent bleach solution to prevent transmission of hantavirus (Mills et al. 1995).

\section{$\underline{\text { Reptile and Amphibian Survey }}$}

Any reptiles and amphibians encountered on site during any field efforts were identified to species and recorded.

\section{Terrestrial Arthropod Survey}

Surveys for Special Status arthropod genera were conducted in appropriate habitats. Surveys occurred concurrently with, or after, bird surveys during the summer of 2010 and spring of 2011. 
Taxa were identified to species where possible, and to genera if not possible. Surveys followed an adapted protocol for butterfly monitoring developed by the Smithsonian Conservation and Research Center (McShea 2008); and were only conducted during optimal conditions of $70^{\circ}$ to $90^{\circ}$ Fahrenheit, little or no wind, and sky conditions greater than 50 percent sunlight. All occurrence locations were recorded with a GPS receiver unit, along with the number of individuals, habitat, weather conditions, and time of observation. Catch-and-release netting by trained Walsh personnel was to be conducted only when a positive identification could not be obtained visually, thus minimizing the risk of injury to the subject. Photographs of detection sites and individuals were obtained, when time permitted, and camera use was proposed by Walsh and not contractually required.

\section{Targeted Wildlife Species}

Targeted surveys were conducted for breeding bird density, nocturnal wildlife (playback for small owls, amphibian call, and bat acoustical), mule deer (in addition to pellet surveys conducted), and carnivores (motion-sensing cameras). Mammalian predator surveys using sifted dirt with a scented predator disc were conducted in the general wildlife surveys. Both the mammalian predator surveys and carnivore camera surveys served to detect predators using two different techniques.

\section{Breeding Bird Density Surveys}

Data on breeding birds were collected during five surveys conducted throughout April and May 2011. The survey protocol used in these transect-based bird surveys incorporated data collection methods necessary to analyze bird density with distance sampling (Buckland et al. 1993), which provides estimates of bird densities and recognizes that not all birds present during the sampling are detected.

Eight 250-meter transects were walked during each of the five visits. Previously established bird survey points (SAIC 2005) were selected as origins for these eight transects. The end points of transects were randomly selected to be evenly spaced within the site, including using some parts of previously established transects (Figure 2). All bird species seen or heard along each transect were recorded, and their distance from the transect line was estimated to the nearest meter using a calibrated Bushnell laser rangefinder. The sighting angle was measured from the transect line with a protractor mounted to a clipboard. Sampling occurred for 3.5 hours after sunrise. Sampling was not conducted in inclement weather.

Program DISTANCE (Thomas et al. 2010) was used to generate bird density estimates. Species' detection function was modeled, based on exact distance values, using the robust models suggested by Buckland et al. (2001). A key function (half-normal, hazard, or uniform) with possible adjustment terms (cosine or polynomial) were used to model the detection function. The best model was then selected using Akaike's information criterion (AIC), and by inspecting probability density functions and Chi-squared Goodness-of-Fit statistics (Buckland et al.1993).

Densities could only be generated for five individual species due to inadequate observation numbers for other species. In addition, the breeding bird data were analyzed in program DISTANCE as clusters, rather than individuals, as advised by Buckland et al. (2001). A cluster is defined as more than one individual at the same location. For example, a pair of American robins (Turdus migratorius) is considered a cluster of two. Also to remain consistent with distance sampling methods, flyovers were not analyzed. An individual is considered a flyover when it was in the air when first observed. 


\section{Nocturnal Surveys}

Nocturnal surveys were conducted for owls, amphibians, and bats. Survey locations are shown in Figure 2. Nocturnal playback surveys for owls followed a protocol adapted from the U.S. Forest Service (Francis and Bradstreet 1997) for species likely to occur at STM. Playback stations were located in potential eastern screech-owl (Megascops asio) habitat. After discussions with Steve Jones, the coordinator for the metro area portion (Region 16) of the Colorado Breeding Bird Atlas II, Great horned owls (Bubo virginianus) were not considered for surveys as this commonly occurring species is widely distributed in Colorado and expected for the site. Playback/response surveys were conducted on March 9 and 10, 2011, corresponding to the regional peak period of calling activity for eastern screech-owls. Playback/response involved playing 30 -seconds of a recorded call of the species followed by 30 -second intervals of listening, repeated for a total of ten minutes at each point.

Amphibian call surveys, following protocol from the U.S. Geological Survey (Droege 2010), occurred on June 7 and 9, 2010. Surveys were conducted along a series of predetermined stops near wetlands, 30 to 60 minutes after sunset. After a minute of waiting to reduce disturbance, observers started a three- to five-minute listening period. The observers recorded all species heard vocalizing. Each record included a calling index value adapted for use across North America: $0=$ no frogs of a given species can be heard calling; $1=$ individual calls, not overlapping; $2=$ calls are overlapping; but individuals are still distinguishable; 3 = numerous frogs can be heard, chorus is constant and overlapping.

Walsh's acoustical bat monitoring specialists conducted handheld acoustical surveys for bats using AnaBat ${ }^{\mathrm{TM}}$ bat detectors. During surveys, hand-held detectors recorded sonograms of any bats detected. Bat surveys occurred concurrently with amphibian call surveys June 7 and 9, 2010.

\section{Mule Deer Surveys}

Results of this survey augmented the sampling conducted for large mammal surveys by adding summer and winter surveys for mule deer (Odocoileus hemionus) use and relative abundance. Mule deer seasonal use surveys were conducted in the summer and winter. The protocol involve walking the entire site one day each in July 2010 and December 2010 using the previously established six large mammal survey transects, as well as areas in-between, walked to access the next transect (Figure 2). In addition, other areas of mule deer use were included in the survey, as appropriate based on observed activity. Field-map data sheets with aerial coverage of the site were used to document their location and numbers during each survey.

\section{Carnivore Camera Surveys}

Walsh used five Trailmaster motion-detecting camera setups with monitors for the carnivore surveys. The monitors are passive sensing units that detect infrared and microwave wave frequencies. Monitors record all interruption incidents of a cone-shaped beam and trigger the camera. Surveys occurred in each of the four seasons: June, September, and December 2010, and April 2011. Five survey locations were selected based on potential carnivore high use areas as evaluated by topography, tracks observed, vegetation, and staff knowledge (Figure 2). The equipment was attached to existing trees using non-permanent straps and clamps. Each survey took place over four consecutive nights. Camera film was developed to assess what animals, including carnivores, were present on the site. Mammalian predator track surveys were also conducted as part of the general wildlife species surveys, and together these two methods gave insight into carnivore presence and use. 


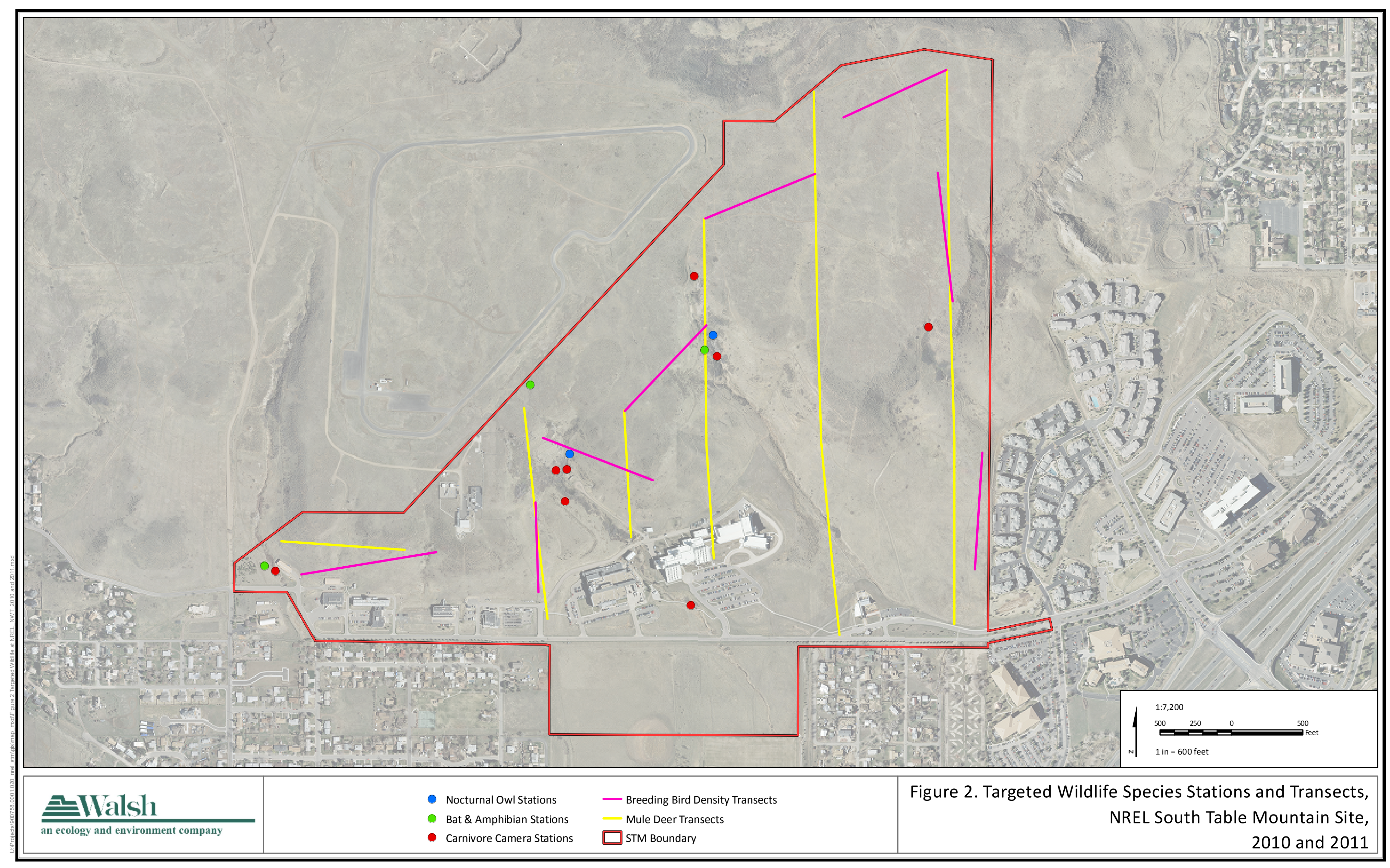

This report is available at no cost from the National Renewable Energy Laboratory (NREL) at www.nrel.gov/publications 


\section{RESULTS}

\section{Vegetation Mapping}

The majority of vegetation at STM belongs to the grassland community type. Within that association, there are two distinct community types: short-grass grassland on the mesa top and mixed-grass grassland located on the slopes and toe area. Other mapped vegetation communities at STM include ravine shrubland, tall shrubland, short shrubland, and wetlands. The plant communities are described below and mapped as illustrated in Figure 3.

\section{Grasslands}

Grasslands are the predominant habitat on the STM site. In general, the grassland areas throughout the site have similar species composition. A list of the plant species found in the STM grasslands is located in Table 1 of Appendix A.

\section{Short-grass grassland}

The short-grass grassland is found on the flat top of the mesa. The dominant grass species are blue grama (Chondrosum gracile), a native prairie species and cheatgrass (Anisantha tectorum), a noxious weed (Photo 1). Populations of diffuse knapweed (Acosta diffusa) and Dalmatian toadflax (Linaria genistifolia subsp. dalmatica) are scattered throughout the whole community. These two noxious weeds comprise approximately one percent of the short-grass. Alyssum (Alyssum parviflorum), an introduced species, is the dominant forb. Several species of prickly pear cactus (Opuntia fragilis, O. macrorhiza, O. phaeacantha, and O. polyacantha) occur throughout the shortgrass mesa top as well as hen-and-chicks (Echinocereus viridiflorus) and pincushion cacti (Coryphantha missouriensis and C. vivipara var. vivpara). Well-draining hillocks (small hills) often support thick stands of needle-and-thread grass (Hesperostipa comata) and yucca (Yucca glauca). Some short shrubs such as rubber rabbitbrush (Chrysothamnus nauseosus subsp. graveolens), chokecherry (Padus virginiana), and skunkbrush (Rhus aromatica subsp. trilobata) occur infrequently in the shortgrass area and concentrate along the rimrock areas as do several of large hackberry trees (Celtis reticulata) at the very edge of the mesa top.

\section{Mixed-grass grassland}

The mesa slopes and toe areas on the STM site also support blue grama and cheatgrass but are dominated by a mixed-grass species association of needle-and-thread grass and western wheatgrass (Pascopyrum smithii), with smaller amounts of big bluestem (Andropogon gerardii), side-oats grama (Bouteloua curtipendula), three-awn (Aristida purpurea), and green needle grass (Nassella viridula) (Photo 2). As in the short-grass areas, a large number of forbs also occur in the mixed-grass grassland.

A few patches of anomalous vegetation occur within the mixed-grassland, where subsurface water appears to be closer to the surface than in most of the area. These areas support wide swaths of mat muhly (Muhlenbergia richardsonis). One is located on a southern-facing slope, near the eastern property boundary. The other is located on a southwestern-facing slope of the ravine north of the Visitor Center. This area is notable for a large population of poison ivy (Toxicodendron rydbergii), which grows in thickets of tall (one meter and larger) plants that have a woody, shrublike, growth form. A small number of plains cottonwood (Populus deltoides) saplings, skunkbrush, chokecherry, and snowberry (Symphoricarpos occidentalis) occur in this patch as well.

The mixed-grass areas grade into both the upland and ravine shrublands and contribute the majority of the understory in these areas. Some mixed-grass areas also blend into disturbed areas, 
where reclamation species such as crested wheatgrass (Agropyron cristatum) and smooth brome (Bromopsis inermis) have been planted and have subsequently spread into the mixed-grass community. Crested wheatgrass and smooth brome tend to outcompete native species, form monocultures, and diminish habitat diversity. These two grass species were widely used, historically, in reclamation efforts. However, native grass seeding mixes are now used which no longer include these invasive grass species.

\section{Upland Shrublands}

Shrubland habitat occurs along the upper sides of ravines, and on the steeper mesa slopes, becoming more prominent as elevation increases up to the top of the mesa. The upland shrubland habitat, which excludes the shrublands in the ravine bottoms, comprises tall shrubland and short shrubland communities, very similar in overall composition but distinguished by the dominant species. Tall shrublands were dominated by mountain-mahogany (Cercocarpus montanus) and cheatgrass and short shrublands were dominated by rubber rabbitbrush and skunkbrush. A list of the plant species found in the STM shrublands is located in Table 2 of Appendix A.

\section{Tall Shrubland}

The tall shrubland areas are defined by stands of mountain-mahogany that occur along the rim of the mesa, usually where volcanic cap rock is exposed, and on the upper mesa slopes below rimrock areas (Photo 3). The understory is notably sparse throughout this community, with a large amount of bare soil. Cheatgrass is the most common herbaceous species in these areas, intermixed with needle-and-thread grass, yucca, and many cacti.

\section{Short Shrubland}

The short shrublands occur on elevated flat areas amidst the surrounding grasslands, some of which appear to have experienced surficial disturbance in the past (Photo 4). These areas are distinctive because of their dominance by rubber rabbitbrush. The other common location for short shrublands is on the outer slopes of the ravines. Skunkbrush defines these and other short shrublands along the upper portions of the steepest slopes of the mesa. These communities usually grade into the ravine shrublands along the drainage bottoms and the tall shrublands near the top of the mesa slopes. The short shrubland community also has a sparse understory of the same grasses and forbs as the tall shrub community.

\section{Ravine Shrublands}

Ravine shrublands are limited to the lower sides and bottoms of the drainages that cut down through the mesa slopes (Photos 5 and 6). These communities support a variety of shrubs such as skunkbrush, chokecherry, and wild plum (Prunus americana), often growing in dense, impassible thickets. A few plains cottonwoods and peach-leaf willow (Salix amygdaloides) trees occur at the top of the ravine channels and in other portions of the channel where the subsurface water table appears to be relatively high. A diverse herbaceous component is found in these drainages. In one instance near the southeast site boundary, a ravine shrubland grades into an ephemeral drainage at the toe of the mesa. This drainage is vegetated with grassland species and contains only occasional surface water run-off. A list of the plant species found in the ravine shrublands is located in Table 3 of Appendix A.

\section{$\underline{\text { Wetlands }}$}

Five very small communities on the STM site were found to support wetland vegetation. These communities were not examined for the soils and hydrology that would classify them as functioning wetlands; rather they are noted only for their domination by wetland vegetation. 
These are limited to very small areas (less than half an acre in total). One is in a shallow swale at the mouth of the ravine at the southwestern corner of the project boundary (Figure 3) where surface water and/or subsurface drainage have created a pocket of saturated soil. Species here include sedges (Carex spp.), rushes (Juncus spp.), bulrush (Schoenoplectus sp.), and peach-leaf willow (Photos 7 and 8). The second wetland could have formed as a result of past construction activities. This linear depression supports wetland vegetation along the central portion of the western site boundary, northeast of the Solar Industrial Mesa Test Area. Perhaps where equipment was once staged, this area appears to hold seasonal water for enough consecutive growing seasons to support some wetland vegetation including arctic rush (Juncus arcticus), American speedwell (Veronica americana), and broadleaf cattail (Typha latifolia) (Photo 9). A list of the plant species found in the wetlands is located in Table 4 of Appendix A.

The wetland at the mouth of the ravine may no longer experience the hydrology that originally allowed these plants to establish there. In 2002, this plant community supported populations of cattails that were not observed in 2010.

Three small seeps are located on the hill slope between the Visitor Center and the public trail on the far eastern boundary of the site. These seeps are dominated with sedges, rushes, and Canada thistle (Breea arvensis).

A seventh wetland community that was observed by Plantae (2002), but no longer appears to support wetland vegetation. In 2002, cattail species near an old stocktank in the eastern-most drainage appeared to have been suppored by a pipe coming out of the hillside. Although the stocktank was observed in 2010, it appears the cattails have not persisted in the intervening years.

\section{Disturbed/Reclaimed}

This habitat type comprises all of the areas at the site that have experienced surface disturbance to vegetation caused by human activities (Photo 10). These mostly occur on the perimeter of the buildings, roads, parking lots, and soil dumping areas. Most of these areas appear to have been revegetated and support a combination of native grassland plants, planted ornamental revegetation species, and native and introduced weeds. The former Forest Service tree farm in the southwestern corner of the site was included in this habitat type because the surface has been disturbed in the past and a number of non-native trees still persist in this area. A list of the plant species found in the disturbed/reclaimed community is located in Table 5 of Appendix A.

\section{Developed}

Developed areas comprise the human-constructed, non-vegetated portions of the site. These include parking lots, buildings, and paved roads. Developed areas do not constitute plant communities and are therefore not listed in Appendix A. However, these areas also support trees, shrubs, and herbaceous plants including grasses and forbs. 


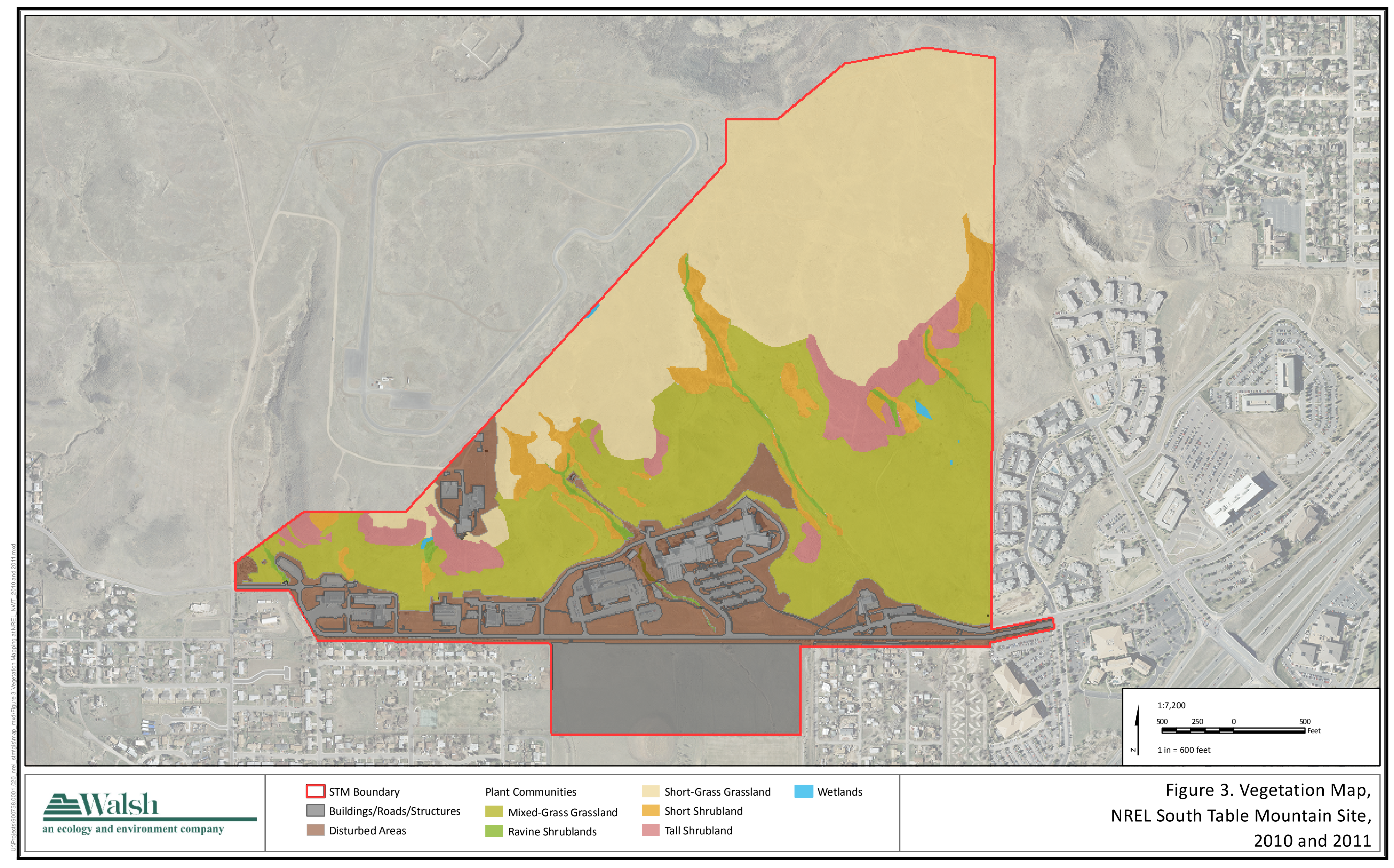

This report is available at no cost from the National Renewable Energy Laboratory (NREL) at www.nrel.gov/publications 


\section{Noxious Weeds}

Weed species that occur on the STM site are listed in Table 1. Mapped weed populations are illustrated in Figure 4. The noxious weeds found on the site vary from small isolated populations to species that are widespread and dominate entire plant communities. Myrtle spurge (Euphorbia myrsinites), common teasel (Dipsacus fullonum), whitetop (Cardaria draba), Russian-olive (Elaeagnus angustifolia), and scotch thistle (Onopordum acanthium) occur in single or isolated populations. By comparison, dalmation toadflax and diffuse knapweed are found throughout the site, frequently occurring as single individuals dotting the landscape. Canada thistle and hound's tongue (Cynoglossum officinale) are routinely found in each of the drainages.

Cheatgrass is found in large populations throughout the entire site and completely dominates the mesa top. This noxious weed has significantly reduced plant diversity and has notably crowded out the short-grass grassland species.

Table 1. Noxious Weed Species Identified at the NREL South Table Mountain Site, June 2010

\begin{tabular}{|l|c|c|}
\hline \multicolumn{1}{|c|}{ Common Name } & Scientific Binomial & $\begin{array}{c}\text { Estimated Area } \\
\text { (acres) }\end{array}$ \\
\hline Canada thistle & Breea arvensis & * \\
\hline Cheatgrass & Anisantha tectorum & 0.06 \\
\hline Common teasel & Dipsacus fullonum & 1.3 \\
\hline Dalmatian toadflax & Linaria genistifolia subsp. & 1.1 \\
\hline Diffuse knapweed & Acolmatica & 0.4 \\
\hline Field bindweed & Convolvulus arvensis & 0.001 \\
\hline Hound's tongue & Cynoglossum officinale & 2.1 \\
\hline Musk thistle & Carduus nutans & 0.1 \\
\hline Myrtle spurge & Euphorbia myrsinites & 0.04 \\
\hline Russian-olive & Elaeagnus angustifolia & 0.03 \\
\hline Scotch thistle & Onopordum acanthium & 0.1 \\
\hline Whitetop & Cardaria draba & \\
\hline
\end{tabular}

Species in bold font are on the list of top ten priority-for-control weeds for Colorado.

${ }^{*}$ Cheatgrass was pervasive throughout the site and was not specifically mapped. **Diffuse knapweed was found throughout the site and estimated area is for higher densities of plants per square meter. 


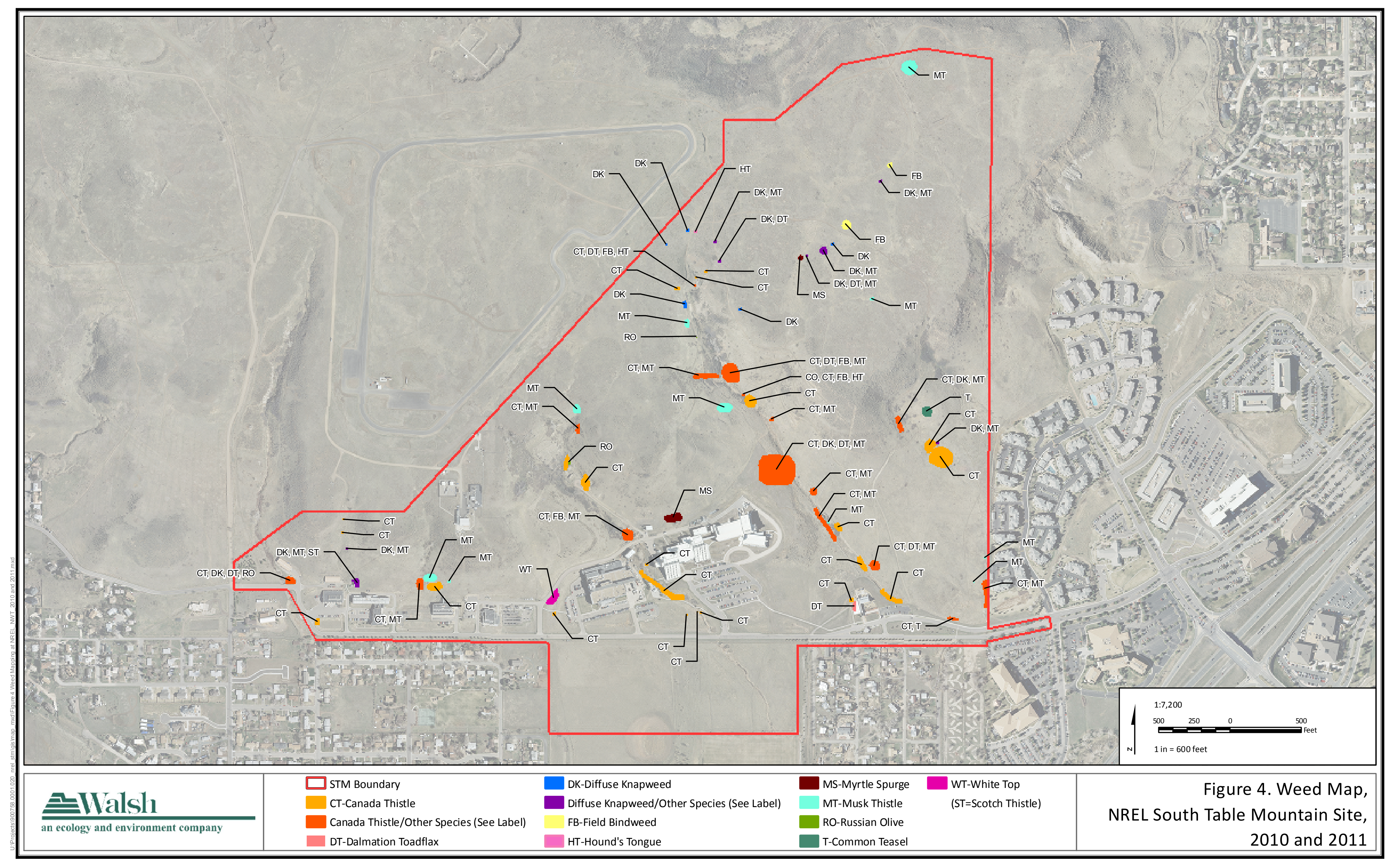

This report is available at no cost from the National Renewable Energy Laboratory (NREL) at www.nrel.gov/publications 


\section{Wildlife Survey Results}

Thirteen different types of wildlife surveys were conducted over a period of 12 months at STM.

Table 2 summarizes the seasons during which each survey was conducted.

Table 2. Wildlife Survey Seasons, NREL South Table Mountain Site, 2010-2011

\begin{tabular}{|c|c|c|c|c|c|}
\hline \multirow[b]{2}{*}{ Survey Type } & \multicolumn{5}{|c|}{ Season } \\
\hline & $\begin{array}{c}\text { Summer } \\
2010 \\
\end{array}$ & $\begin{array}{c}\text { Fall } \\
2010\end{array}$ & $\begin{array}{c}\text { Winter } \\
2010-2011 \\
\end{array}$ & $\begin{array}{c}\text { Spring } \\
2011 \\
\end{array}$ & $\begin{array}{c}\text { Summer } \\
2011 \\
\end{array}$ \\
\hline Bird & $x$ & $x$ & $\mathrm{X}$ & $x$ & \\
\hline Raptor & & $\mathrm{X}$ & & $\mathrm{X}$ & \\
\hline Large Mammal & & & & $x$ & \\
\hline Mammalian Predator & $x$ & $x$ & $x$ & $x$ & \\
\hline Small Mammal & $\mathrm{X}$ & & & & \\
\hline Reptile and Amphibian & $x$ & $x$ & $x$ & $x$ & $x$ \\
\hline Terrestrial Arthropod & $\mathrm{X}$ & $\mathrm{X}$ & $\mathrm{X}$ & $\mathrm{X}$ & $\mathrm{X}$ \\
\hline Breeding Bird Density & & & & & $\mathrm{X}$ \\
\hline Nocturnal Owl & & & & $x$ & \\
\hline Nocturnal Amphibian & $X$ & & & & \\
\hline Acoustical Bat & $x$ & & & & \\
\hline Mule Deer & $x$ & & $x$ & & \\
\hline Carnivore Camera & $x$ & $\mathrm{X}$ & $\mathrm{X}$ & $x$ & \\
\hline
\end{tabular}

Table 3 summarizes all wildlife observations, as well as those from 1987 (FORUM 1987) and 2004/2005 (SAIC 2005), regardless of sampling regime, and includes incidental observations.

In 2010/2011 a total of 102 species were observed for all wildlife surveys conducted on STM. This includes five species of herptiles, 86 species of birds, and 11 species of mammals.

Comparisons to earlier surveys are addressed in the discussion. 


\section{Table 3. Wildlife Survey Results, NREL South Table Mountain Site, 2010-2011}

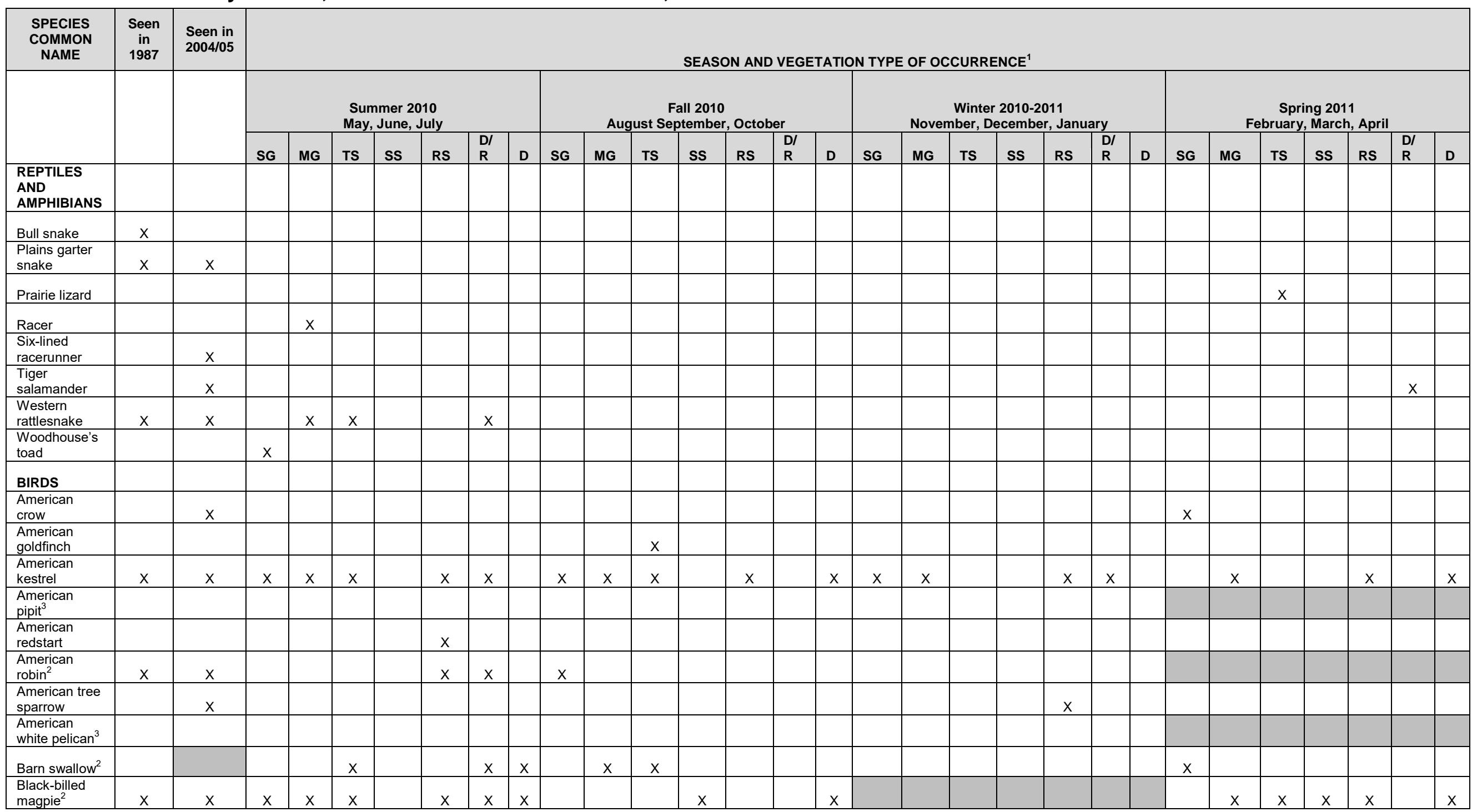




\begin{tabular}{|c|c|c|c|c|c|c|c|c|c|c|c|c|c|c|c|c|c|c|c|c|c|c|c|c|c|c|c|c|c|c|}
\hline \multirow[t]{3}{*}{$\begin{array}{c}\text { SPECIES } \\
\text { COMMON } \\
\text { NAME }\end{array}$} & \multirow[t]{3}{*}{$\begin{array}{c}\text { Seen } \\
\text { in } \\
1987\end{array}$} & $\begin{array}{l}\text { Seen in } \\
2004 / 05\end{array}$ & \multicolumn{28}{|c|}{ SEASON AND VEGETATION TYPE OF OCCURRENCE ${ }^{1}$} \\
\hline & & & \multicolumn{7}{|c|}{$\begin{array}{l}\text { Summer } 2010 \\
\text { May, June, July }\end{array}$} & \multicolumn{7}{|c|}{$\begin{array}{l}\text { Fall } 2010 \\
\text { August September, October }\end{array}$} & \multicolumn{7}{|c|}{$\begin{array}{l}\text { Winter 2010-2011 } \\
\text { November, December, January }\end{array}$} & \multicolumn{7}{|c|}{$\begin{array}{l}\text { Spring } 2011 \\
\text { February, March, April }\end{array}$} \\
\hline & & & SG & MG & TS & ss & RS & $\begin{array}{l}\mathrm{D} / \\
\mathrm{R}\end{array}$ & D & SG & MG & TS & ss & RS & $\begin{array}{l}\mathrm{D} / \\
\mathrm{R}\end{array}$ & D & SG & MG & TS & ss & RS & $\begin{array}{l}\mathrm{D} / \\
\mathrm{R}\end{array}$ & D & SG & MG & TS & ss & RS & $\begin{array}{l}\mathrm{D} / \\
\mathrm{R}\end{array}$ & D \\
\hline $\begin{array}{l}\text { Black-capped } \\
\text { chickadee }\end{array}$ & & $x$ & & & & & & & & & & & & & & & & & & & & & & & & & & & & \\
\hline $\begin{array}{l}\text { Black- } \\
\text { crowned night } \\
\text { heron }\end{array}$ & & $x$ & & & & & & & & & & & & & & & & & & & & & & & & & & & & \\
\hline $\begin{array}{l}\text { Black-headed } \\
\text { grosbeak }^{2}\end{array}$ & & & & & & & & & & & & & & & & & & & & & & & & & & & & $x$ & & \\
\hline $\begin{array}{l}\text { Blue-gray } \\
\text { gnatcatcher }^{2}\end{array}$ & & & & & $x$ & & & $x$ & & & & & & & & & & & & & & & & & & $\mathrm{x}$ & & $\mathrm{x}$ & & \\
\hline Blue jay & & $x$ & & & & & & & $\mathrm{x}$ & & & & & & & $x$ & & & & & & & & & & & & & & \\
\hline $\begin{array}{l}\text { Brewer's } \\
\text { blackbird }^{2}\end{array}$ & $x$ & & & & & & & & & & & & & & & $x$ & & & & & & & & $x$ & & & & & & \\
\hline $\begin{array}{l}\text { Brewer's } \\
\text { sparrow }^{2}\end{array}$ & & & & & & & & & & $\mathrm{x}$ & $x$ & $\mathrm{x}$ & & & & & & & & & & & & & & & & $\mathrm{x}$ & & $\mathrm{x}$ \\
\hline $\begin{array}{l}\text { Broad-tailed } \\
\text { hummingbird }^{2}\end{array}$ & & & & & & & & & & $x$ & & $x$ & & & & & & & & & & & & & & & & & & \\
\hline $\begin{array}{l}\text { Broad-winged } \\
\text { hawk }^{2}\end{array}$ & & & & & & & & & & & & & & & & & & & & & & & & & & & & $\mathrm{x}$ & & \\
\hline $\begin{array}{l}\text { Brown-headed } \\
\text { cowbird }^{2}\end{array}$ & $\mathrm{x}$ & $x$ & & & $x$ & & $x$ & $x$ & $\mathrm{x}$ & & & & & & & & & & & & & & & & & $x$ & & $\mathrm{x}$ & & $x$ \\
\hline $\begin{array}{l}\text { Bullock's } \\
\text { oriole }^{2}\end{array}$ & & $\mathrm{x}$ & $\mathrm{x}$ & $\mathrm{x}$ & & $\mathrm{x}$ & $\mathrm{x}$ & $\mathrm{x}$ & $\mathrm{x}$ & & & & & & & & & & & & & & & & $\mathrm{x}$ & & & $\mathrm{x}$ & & \\
\hline Bushtit $^{3}$ & & & & & & & & & & & & $\mathrm{x}$ & & & & & & & & & & & & & & & & & & \\
\hline California gull & & $x$ & & & & & & & & & & & & & & & & & & & & & & & & & & & & \\
\hline Canada goose & & $\mathrm{x}$ & & & & & & & & & & & & & & & $x$ & & & & & & & & & & & & & \\
\hline $\begin{array}{l}\text { Cedar } \\
\text { waxwing }\end{array}$ & & & & & $x$ & & & & & & & & & & & & & & & & & & & & & & & & & \\
\hline $\begin{array}{l}\text { Cassin's } \\
\text { kingbird }^{3}\end{array}$ & & & & & & & & & & & & $\mathrm{x}$ & & & & & & & & & & & & & & & & & & \\
\hline $\begin{array}{l}\text { Chestnut- } \\
\text { collared } \\
\text { longspur }\end{array}$ & & & & & & & & & & $\mathrm{x}$ & & & & & & & & & & & & & & & & & & & & \\
\hline $\begin{array}{l}\text { Chipping } \\
\text { sparrow }\end{array}$ & & & & & & & & & & $\mathrm{x}$ & $\mathrm{x}$ & & & $x$ & & $x$ & & & & & & & & & & $\mathrm{x}$ & & & & \\
\hline Cliff swallow² & & & $x$ & & & & & & & & & & & & & & & & & & & & & & & & & & & \\
\hline $\begin{array}{l}\text { Common } \\
\text { grackle }\end{array}$ & & & & & & & & & $x$ & & & & & & & & & & & & & & & $x$ & & & & & & $x$ \\
\hline
\end{tabular}




\begin{tabular}{|c|c|c|c|c|c|c|c|c|c|c|c|c|c|c|c|c|c|c|c|c|c|c|c|c|c|c|c|c|c|c|}
\hline \multirow[t]{3}{*}{$\begin{array}{l}\text { SPECIES } \\
\text { COMMON } \\
\text { NAME }\end{array}$} & $\begin{array}{c}\text { Seen } \\
\text { in } \\
1987\end{array}$ & $\begin{array}{l}\text { Seen in } \\
2004 / 05\end{array}$ & \multicolumn{28}{|c|}{ SEASON AND VEGETATION TYPE OF OCCURRENCE ${ }^{1}$} \\
\hline & & & \multicolumn{7}{|c|}{$\begin{array}{l}\text { Summer } 2010 \\
\text { May, June, July }\end{array}$} & \multicolumn{7}{|c|}{$\begin{array}{l}\text { Fall } 2010 \\
\text { August September, October }\end{array}$} & \multicolumn{7}{|c|}{$\begin{array}{l}\text { Winter 2010-2011 } \\
\text { November, December, January }\end{array}$} & \multicolumn{7}{|c|}{$\begin{array}{l}\text { Spring } 2011 \\
\text { February, March, April }\end{array}$} \\
\hline & & & SG & MG & TS & ss & RS & $\begin{array}{l}\mathrm{D} I \\
\mathrm{R}\end{array}$ & D & SG & MG & TS & ss & RS & $\begin{array}{l}\mathrm{D} I \\
\mathrm{R}\end{array}$ & D & SG & MG & TS & ss & RS & DI & D & SG & MG & TS & ss & RS & $\begin{array}{l}\text { DI } \\
\text { R }\end{array}$ & D \\
\hline $\begin{array}{l}\text { Common } \\
\text { nighthawk }\end{array}$ & $\mathrm{x}$ & $x$ & $x$ & & & & & & & & & & & & & & & & & & & & & & & & & & & \\
\hline $\begin{array}{l}\text { Common } \\
\text { raven }^{2}\end{array}$ & & $x$ & & & & & & & & & & & & & & & & & & & & & & & $x$ & & & & & \\
\hline Wilson's snipe & & $x$ & & & & & & & & & & & & & & & & & & & & & & & & & & & & \\
\hline $\begin{array}{l}\text { Cooper's } \\
\text { hawk }\end{array}$ & & $x$ & & & & & & & & & & $\mathrm{x}$ & & & & $x$ & & & & & & & & & & & & & & \\
\hline $\begin{array}{l}\text { Dark-eyed } \\
\text { junco }\end{array}$ & & $x$ & & & & & & & & & & & & & & & & & $x$ & & $\mathrm{x}$ & & & & & & & $x$ & & \\
\hline $\begin{array}{l}\text { Double- } \\
\text { crested }^{\text {cormorant }^{2}}\end{array}$ & & & & & & & & & & & & & & & & & & & & & & & & & & & & & & \\
\hline $\begin{array}{l}\text { Downy } \\
\text { woodpecker }\end{array}$ & & & & & & & & & & & & & & & & & & & & & & & & & & & & & & \\
\hline $\begin{array}{l}\text { Eastern } \\
\text { kingbird }\end{array}$ & & & $x$ & $x$ & & & & & & & & & & & & & & & & & & & & & & & & & & \\
\hline $\begin{array}{l}\text { European } \\
\text { starling }^{2}\end{array}$ & $x$ & $x$ & & $x$ & & & $\mathrm{x}$ & & $x$ & & & & & $x$ & & $x$ & & & & & $x$ & & & & $x$ & & & $x$ & & $x$ \\
\hline $\begin{array}{l}\text { Unidentified } \\
\text { flycatcher }\end{array}$ & & $\mathrm{x}$ & & & & & & & & & & & & & & & & & & & & & & & & & & & & \\
\hline Golden eagle & & $x$ & & & & & & & & & & & & & & & & & & & & & & & & & & & & \\
\hline $\begin{array}{l}\text { Grasshopper } \\
\text { sparrow }\end{array}$ & & & $x$ & & & & & & & & & & & & & & & & & & & & & & & & & & & \\
\hline $\begin{array}{l}\text { Great blue } \\
\text { heron }\end{array}$ & & $\mathrm{x}$ & & & & & & & & & & & & & & & & & & & & & & & & & & & & \\
\hline $\begin{array}{l}\text { Green-tailed } \\
\text { towhee }^{2}\end{array}$ & & & & & $x$ & & & & & & & $x$ & $x$ & $\mathrm{x}$ & & & & & & & & & & & & $x$ & & & & \\
\hline $\begin{array}{l}\text { Hepatic } \\
\text { tanager }\end{array}$ & & & & & & & $x$ & & & & & & & & & & & & & & & & & & & & & & & \\
\hline Hermit thrush ${ }^{3}$ & & & & & & & & & & & & & & & & & & & & & & & & & & $x$ & & & & \\
\hline Horned lark ${ }^{2}$ & $x$ & & & & & & & & & & & & & & & & & & & & & & & $x$ & & & & & & \\
\hline House finch ${ }^{2}$ & & $x$ & $x$ & $x$ & & & $x$ & $x$ & $x$ & $x$ & $x$ & $x$ & & $x$ & & $x$ & $x$ & $x$ & $x$ & & $x$ & $x$ & $x$ & & $x$ & $x$ & $x$ & & & $x$ \\
\hline $\begin{array}{l}\text { House } \\
\text { sparrow }^{2}\end{array}$ & & $x$ & & $x$ & & & $x$ & $x$ & $x$ & & & & & & & & & & & & & & $x$ & & & & & & & $x$ \\
\hline House wren ${ }^{2}$ & & & & & & & $\mathrm{x}$ & $\mathrm{x}$ & & & & & & $x$ & & & & & & & & & & & & & & $x$ & & \\
\hline Killdeer $^{2}$ & $x$ & $x$ & $x$ & & & & & & & & & & & & & & & & & & & & & $x$ & & & & & & \\
\hline
\end{tabular}




\begin{tabular}{|c|c|c|c|c|c|c|c|c|c|c|c|c|c|c|c|c|c|c|c|c|c|c|c|c|c|c|c|c|c|c|}
\hline \multirow[t]{3}{*}{$\begin{array}{c}\text { SPECIES } \\
\text { COMMON } \\
\text { NAME }\end{array}$} & $\begin{array}{c}\text { Seen } \\
\text { in } \\
1987\end{array}$ & $\begin{array}{l}\text { Seen in } \\
2004 / 05\end{array}$ & \multicolumn{28}{|c|}{ SEASON AND VEGETATION TYPE OF OCCURRENCE ${ }^{1}$} \\
\hline & & & \multicolumn{7}{|c|}{$\begin{array}{l}\text { Summer } 2010 \\
\text { May, June, July }\end{array}$} & \multicolumn{7}{|c|}{$\begin{array}{c}\text { Fall } 2010 \\
\text { August September, October }\end{array}$} & \multicolumn{7}{|c|}{$\begin{array}{l}\text { Winter 2010-2011 } \\
\text { November, December, January }\end{array}$} & \multicolumn{7}{|c|}{$\begin{array}{c}\text { Spring } 2011 \\
\text { February, March, April }\end{array}$} \\
\hline & & & SG & MG & TS & ss & RS & $\begin{array}{l}\mathrm{D} I \\
\mathrm{R}\end{array}$ & D & SG & MG & TS & ss & RS & \begin{tabular}{|l|}
$\mathrm{DI}$ \\
$\mathrm{R}$ \\
\end{tabular} & D & SG & MG & TS & ss & RS & $\begin{array}{l}\mathrm{D} I \\
\mathrm{R} \\
\end{array}$ & D & SG & MG & TS & ss & RS & $\begin{array}{l}\mathrm{D} I \\
\mathrm{R}\end{array}$ & $\mathbf{D}$ \\
\hline Lark bunting & $x$ & $x$ & & & & & & & & & & & & & & & & & & & & & & & & & & & & \\
\hline Lark sparrow $^{2}$ & & & & & & & & & & $x$ & & & & & & & & & & & & & & $x$ & & $x$ & & & & \\
\hline Lazuli bunting & & & $\mathrm{x}$ & $x$ & $x$ & & $x$ & & & & & & & $\mathrm{x}$ & & & & & & & & & & & & & & & & \\
\hline $\begin{array}{l}\text { Lesser } \\
\text { goldfinch }\end{array}$ & & & & $\mathrm{x}$ & & & $\mathrm{X}$ & & $x$ & & $x$ & $x$ & & & $\mathrm{x}$ & & & & & & & & & & & & & & & \\
\hline $\begin{array}{l}\text { Loggerhead } \\
\text { shrike }^{2}\end{array}$ & & $\mathrm{x}$ & & & & & & & & & & & & & & & & & & & & & & $\mathrm{x}$ & & $x$ & & & & \\
\hline $\begin{array}{l}\text { MacGillivray's } \\
\text { warbler }\end{array}$ & & $\mathrm{x}$ & & & & & $\mathrm{x}$ & & & & & & & $\mathrm{x}$ & & & & & & & & & & & & & & & & \\
\hline Mallard $^{2}$ & & $x$ & & & & & & & & & & & & & & & & & & & & & & & $\mathrm{x}$ & & & & & \\
\hline $\begin{array}{l}\text { Mountain } \\
\text { bluebird }^{2} \\
\end{array}$ & $\mathrm{x}$ & $\mathrm{x}$ & $\mathrm{x}$ & & & & & & & & & & & & & & & & & & & & & & & & & & & \\
\hline $\begin{array}{l}\begin{array}{l}\text { Mountain } \\
\text { chickadee }^{3}\end{array} \\
\end{array}$ & & & & & & & & & & & & & & & & & & & & & & & & & & & & & & \\
\hline $\begin{array}{l}\text { Mourning } \\
\text { dove }^{2}\end{array}$ & $\mathrm{x}$ & $\mathrm{x}$ & $\mathrm{X}$ & $\mathrm{x}$ & $\mathrm{X}$ & & $\mathrm{x}$ & & $\mathrm{x}$ & $\mathrm{x}$ & & $\mathrm{x}$ & $\mathrm{x}$ & $\mathrm{x}$ & & $\mathrm{X}$ & & & & & & & & & $\mathrm{x}$ & & & $\mathrm{x}$ & & $\mathrm{x}$ \\
\hline $\begin{array}{l}\text { Northern } \\
\text { flicker }^{2}\end{array}$ & $\mathrm{x}$ & $\mathrm{X}$ & $x$ & & & & & $x$ & $x$ & $x$ & & & & $\mathrm{X}$ & & & & & & & $\mathrm{X}$ & & $x$ & $\mathrm{x}$ & & & & $x$ & & \\
\hline $\begin{array}{l}\text { Northern } \\
\text { goshawk }\end{array}$ & & & & & & & & & & & & & & & & & & & & & & & & & & & & & & \\
\hline $\begin{array}{l}\text { Northern } \\
\text { harrier }\end{array}$ & & $\mathrm{x}$ & & & & & & & & $\mathrm{x}$ & & $\mathrm{x}$ & & & & & & & & & & & & & & & & & & \\
\hline $\begin{array}{l}\text { Northern } \\
\text { Mockingbird }^{5} \\
\end{array}$ & & & & & & & & & & & & & & & & & & & & & & & & & & & & & & $\mathrm{x}$ \\
\hline Osprey & & $\mathrm{x}$ & & & & & & & & & & & & & & & & & & & & & & & & & & & & \\
\hline $\begin{array}{l}\text { Peregrine } \\
\text { falcon }^{4}\end{array}$ & & & & & & & & & & & & & & & & & & & & & & & & & & & & & & \\
\hline Pine siskin & & & & & & & & & & & & & & $\mathrm{x}$ & & & & & & & & & & & & & & & & \\
\hline Prairie falcon & & $\mathrm{x}$ & & & & & & & & & & $x$ & & & & & & & & & $\mathrm{x}$ & & & & & & & & & \\
\hline $\begin{array}{l}\text { Red-breasted } \\
\text { nuthatch }\end{array}$ & & $\mathrm{x}$ & & & & & & & & & & & & & & & & & & & & & & & & & & & & \\
\hline $\begin{array}{l}\text { Red-tailed } \\
\text { hawk }\end{array}$ & $\mathrm{x}$ & $\mathrm{X}$ & & & & & $\mathrm{X}$ & & & & & & & & & & & & & & & & & & & & & & & \\
\hline $\begin{array}{l}\text { Red-winged } \\
\text { blackbird }^{2}\end{array}$ & $\mathrm{x}$ & & & & & & & & & & & & & & & & & & & & & & & $\mathrm{x}$ & & & & $\mathrm{x}$ & & \\
\hline
\end{tabular}




\begin{tabular}{|c|c|c|c|c|c|c|c|c|c|c|c|c|c|c|c|c|c|c|c|c|c|c|c|c|c|c|c|c|c|c|}
\hline \multirow[t]{3}{*}{$\begin{array}{l}\text { SPECIES } \\
\text { COMMON } \\
\text { NAME }\end{array}$} & \multirow[t]{3}{*}{$\begin{array}{c}\text { Seen } \\
\text { in } \\
1987\end{array}$} & \multirow[t]{3}{*}{$\begin{array}{l}\text { Seen in } \\
2004 / 05\end{array}$} & \multicolumn{28}{|c|}{ SEASON AND VEGETATION TYPE OF OCCURRENCE ${ }^{1}$} \\
\hline & & & \multicolumn{7}{|c|}{$\begin{array}{l}\text { Summer } 2010 \\
\text { May, June, July }\end{array}$} & \multicolumn{7}{|c|}{$\begin{array}{c}\text { Fall } 2010 \\
\text { August September, October }\end{array}$} & \multicolumn{7}{|c|}{$\begin{array}{c}\text { Winter 2010-2011 } \\
\text { November, December, January }\end{array}$} & \multicolumn{7}{|c|}{$\begin{array}{c}\text { Spring } 2011 \\
\text { February, March, April }\end{array}$} \\
\hline & & & SG & MG & TS & ss & RS & $\begin{array}{l}\mathbf{D} / \\
\mathbf{R} \\
\end{array}$ & D & SG & MG & TS & ss & RS & $\begin{array}{l}\mathrm{D} / \\
\mathrm{R} \\
\end{array}$ & D & SG & MG & TS & ss & RS & $\begin{array}{l}\mathrm{D} / \\
\mathrm{R} \\
\end{array}$ & D & SG & MG & TS & ss & RS & \begin{tabular}{|l|}
$\mathrm{D} I$ \\
$\mathrm{R}$ \\
\end{tabular} & $\mathbf{D}$ \\
\hline Rock pigeon ${ }^{2}$ & & $\mathrm{x}$ & & $x$ & & & & & $x$ & & & $\mathrm{x}$ & & & & $x$ & & & & & & & $x$ & & & & & & & $\mathrm{x}$ \\
\hline Rock wren ${ }^{2}$ & & $x$ & & & & & & & & $x$ & $\mathrm{x}$ & $x$ & $x$ & $x$ & & & & & & & & & & & & $x$ & $x$ & & & \\
\hline $\begin{array}{l}\text { Ruby-crowned } \\
\text { kinglet }\end{array}$ & & & & & & & & & & & & & & & & & & & & & & & & & & & & $\mathrm{x}$ & & \\
\hline Sage thrasher & & & & & & & & & & $\mathrm{x}$ & & $\mathrm{x}$ & & & & & & & & & & & & & & & $x$ & & & \\
\hline Say's phoebe ${ }^{2}$ & & $\mathrm{x}$ & & $\mathrm{x}$ & & $x$ & & & $x$ & $\mathrm{x}$ & $x$ & & & $\mathrm{x}$ & & & & & & & & & & $\mathrm{x}$ & $\mathrm{x}$ & & & $\mathrm{x}$ & $\mathrm{x}$ & $\mathrm{x}$ \\
\hline $\begin{array}{l}\text { Sharp-shinned } \\
\text { hawk }\end{array}$ & & & & & & & & & & & & & & & & & & & & & & & & & & $x$ & $\mathrm{x}$ & & & $\mathrm{x}$ \\
\hline $\begin{array}{l}\text { Spotted } \\
\text { towhee }^{2}\end{array}$ & & $\mathrm{x}$ & & & $\mathrm{x}$ & $\mathrm{x}$ & $\mathrm{x}$ & & & & & & $\mathrm{x}$ & $\mathrm{x}$ & & & & & & & & & & & & $\mathrm{x}$ & & & & \\
\hline $\begin{array}{l}\text { Swainson's } \\
\text { hawk }^{4}\end{array}$ & & $x$ & & & & & & & & & & & & & & & & & & & & & & & & & & & & \\
\hline Tree swallow & & & & & & & & & & & & & & & & & & & & & & & & & & & & & & \\
\hline $\begin{array}{l}\text { Turkey } \\
\text { vulture }^{2}\end{array}$ & & $x$ & & & & & & & & & & & & & & & & & & & & & & & & & & & & \\
\hline $\begin{array}{l}\text { Unidentified } \\
\text { Buteo sp. }{ }^{4}\end{array}$ & & & & & & & & & & & & & & & & & & & & & & & & & & & & & & \\
\hline $\begin{array}{l}\text { Unidentified } \\
\text { falcon sp. }\end{array}$ & & & & & & & & & & & & & & & & & & & & & & & & & & & & & & \\
\hline $\begin{array}{l}\text { Unidentified } \\
\text { sparrow sp. }\end{array}$ & & $x$ & & & & & & & & & & & & & & & & & & & & & & & & & & & & \\
\hline $\begin{array}{l}\text { Unidentified } \\
\text { sparrow sp. }\end{array}$ & & $\mathrm{x}$ & & & & & & & & & & & & & & & & & & & & & & & & & & & & \\
\hline $\begin{array}{l}\text { Unidentified } \\
\text { species }\end{array}$ & & & & & & & & & & & & & & & & & & & & & & & & & & & & & & \\
\hline $\begin{array}{l}\text { Unidentified } \\
\text { warbler }\end{array}$ & & & & & & & & & & & & & & & & & & & & & & & & & & & & & & \\
\hline $\begin{array}{l}\text { Vesper } \\
\text { sparrow }^{2}\end{array}$ & & $\mathrm{x}$ & $x$ & & & & & & & $\mathrm{x}$ & & $\mathrm{x}$ & $\mathrm{x}$ & & & & & & & & & & & $\mathrm{x}$ & & $x$ & $\mathrm{x}$ & & & \\
\hline $\begin{array}{l}\text { Virginia's } \\
\text { warbler }\end{array}$ & & & & & & & & & & & & & & $\mathrm{x}$ & & & & & & & & & & & & $x$ & & & & \\
\hline $\begin{array}{l}\text { Violet-green } \\
\text { swallow }\end{array}$ & & & & & & & $\mathrm{x}$ & & & & & & & & & & & & & & & & & & & & & & & $\mathrm{x}$ \\
\hline $\begin{array}{l}\text { Western } \\
\text { kingbird }^{2}\end{array}$ & $\mathrm{x}$ & $x$ & $\mathrm{x}$ & $x$ & $\mathrm{x}$ & & $\mathrm{x}$ & $\mathrm{x}$ & & & $\mathrm{x}$ & & & & & & & & & & & & & $x$ & $\mathrm{x}$ & & & & & $\mathrm{x}$ \\
\hline $\begin{array}{l}\text { Western } \\
\text { meadowlark }^{2}\end{array}$ & $\mathrm{x}$ & $\mathrm{x}$ & $x$ & $\mathrm{x}$ & & & $\mathrm{x}$ & & & $x$ & $\mathrm{x}$ & $x$ & & & & & & & & & & & & $x$ & $\mathrm{x}$ & $x$ & $x$ & $x$ & & $\mathrm{x}$ \\
\hline
\end{tabular}




\begin{tabular}{|c|c|c|c|c|c|c|c|c|c|c|c|c|c|c|c|c|c|c|c|c|c|c|c|c|c|c|c|c|c|c|}
\hline \multirow[t]{3}{*}{$\begin{array}{l}\text { SPECIES } \\
\text { COMMON } \\
\text { NAME }\end{array}$} & $\begin{array}{c}\text { Seen } \\
\text { in } \\
1987\end{array}$ & $\begin{array}{l}\text { Seen in } \\
2004 / 05\end{array}$ & \multicolumn{28}{|c|}{ SEASON AND VEGETATION TYPE OF OCCURRENCE ${ }^{1}$} \\
\hline & & & \multicolumn{7}{|c|}{$\begin{array}{l}\text { Summer } 2010 \\
\text { May, June, July }\end{array}$} & \multicolumn{7}{|c|}{$\begin{array}{c}\text { Fall } 2010 \\
\text { August September, October }\end{array}$} & \multicolumn{7}{|c|}{$\begin{array}{l}\text { Winter 2010-2011 } \\
\text { November, December, January }\end{array}$} & \multicolumn{7}{|c|}{$\begin{array}{l}\text { Spring } 2011 \\
\text { February, March, April }\end{array}$} \\
\hline & & & SG & MG & TS & SS & RS & $\begin{array}{l}\mathrm{D} / \\
\mathrm{R}\end{array}$ & D & SG & MG & TS & ss & RS & $\begin{array}{l}\mathrm{D} / \\
\mathrm{R}\end{array}$ & D & SG & MG & TS & SS & RS & $\begin{array}{l}\mathrm{D} / \\
\mathrm{R}\end{array}$ & D & SG & MG & TS & SS & RS & $\begin{array}{l}\mathrm{D} / \\
\mathrm{R}\end{array}$ & D \\
\hline $\begin{array}{l}\text { Western } \\
\text { scrub-jay }^{2}\end{array}$ & & $\mathrm{x}$ & & & & & $x$ & & & & & & & $\mathrm{x}$ & & $x$ & & & & & $\mathrm{x}$ & & & & & & & $\mathrm{x}$ & & \\
\hline $\begin{array}{l}\text { White- } \\
\text { crowned }^{2} \\
\text { sparrow }^{2}\end{array}$ & & $\mathrm{x}$ & & & & & & & & & & & & & & & & & & & $\mathrm{X}$ & & & & & & $\mathrm{x}$ & $\mathrm{x}$ & & \\
\hline $\begin{array}{l}\text { White-faced } \\
\text { ibis }^{3}\end{array}$ & & & & & & & & & & & & & & & & & & & & & & & & & & & & & & \\
\hline $\begin{array}{l}\text { White- } \\
\text { throated swift }^{3}\end{array}$ & & & & & & & & & & & & & & & & & & & & & & & & & & & & & & \\
\hline $\begin{array}{l}\text { Yellow- } \\
\text { breasted chat }\end{array}$ & & & & & & & $\mathrm{x}$ & & & & & & & & & & & & & & & & & & & & & & & \\
\hline $\begin{array}{l}\text { Yellow- } \\
\text { rumped } \\
\text { Warbler } \\
\end{array}$ & & & & & & & & & & & & $\mathrm{x}$ & & & & & & & & & & & & $\mathrm{x}$ & & & & & & \\
\hline Mammals & & & & & & & & & & & & & & & & & & & & & & & & & & & & & & \\
\hline $\begin{array}{l}\text { Black-tailed } \\
\text { jackrabbit }\end{array}$ & $x$ & & $\mathrm{x}$ & & & & & & & & & & & & & & & & & & & & & & & & & & & \\
\hline $\begin{array}{l}\text { Bushy-tailed } \\
\text { woodrat }\end{array}$ & $x$ & & & & & & & & & & & & & & & & & & & & & & & & & & & & & \\
\hline Coyote & $x$ & $x$ & $\mathrm{x}$ & & & & & & & $\mathrm{x}$ & & & & & & & $\mathrm{x}$ & $\mathrm{x}$ & & & & & & $x$ & $\mathrm{x}$ & & & & & \\
\hline Deer Mouse & $x$ & & $x$ & & & & $x$ & & & & & & & & & & & & & & & & & $x$ & & & & $\mathrm{x}$ & & \\
\hline Fox squirrel & & $x$ & & & & & & & & & & & & & & & & & & & & & & & & & & & & \\
\hline $\begin{array}{l}\text { Long-tailed } \\
\text { weasel }\end{array}$ & & $\mathrm{X}$ & & & & & & & & & & & & & & & & & & & & & & & & & & & & \\
\hline $\begin{array}{l}\text { Mexican } \\
\text { woodrat }\end{array}$ & & $\mathrm{X}$ & $\mathrm{X}$ & & & & $\mathrm{X}$ & & & & & & & & & & & & & & & & & & & & & $\mathrm{x}$ & & \\
\hline $\begin{array}{l}\text { Mountain } \\
\text { cottontail }\end{array}$ & $x$ & $x$ & & & & & & $\mathrm{x}$ & & & & & & & & & & & $x$ & & & & & & & & & & & $\mathrm{x}$ \\
\hline Mule deer & $x$ & $x$ & $x$ & $\mathrm{x}$ & $x$ & $x$ & $x$ & & & $x$ & & $\mathrm{x}$ & $x$ & $\mathrm{x}$ & & & $x$ & & $x$ & $x$ & $\mathrm{x}$ & & & $x$ & & $\mathrm{x}$ & & $\mathrm{x}$ & & \\
\hline Prairie vole & $x$ & $x$ & & & & & & & & & & & & & & & & & & & & & & & & & & & & \\
\hline Raccoon & $x$ & $x$ & & & & & & & & & & & & $\mathrm{x}$ & & & & & & & & & & & & & & & & \\
\hline Red Fox & $\mathrm{x}$ & & $\mathrm{x}$ & & & & & & $\mathrm{x}$ & $\mathrm{x}$ & $\mathrm{x}$ & $\mathrm{X}$ & & & & & $\mathrm{x}$ & $\mathrm{x}$ & $\mathrm{x}$ & & & & & $\mathrm{x}$ & $\mathrm{x}$ & & & & & \\
\hline Striped skunk & & & & & & & & & & & & & & & & & $x$ & & $x$ & & & & & & & & & & & \\
\hline
\end{tabular}




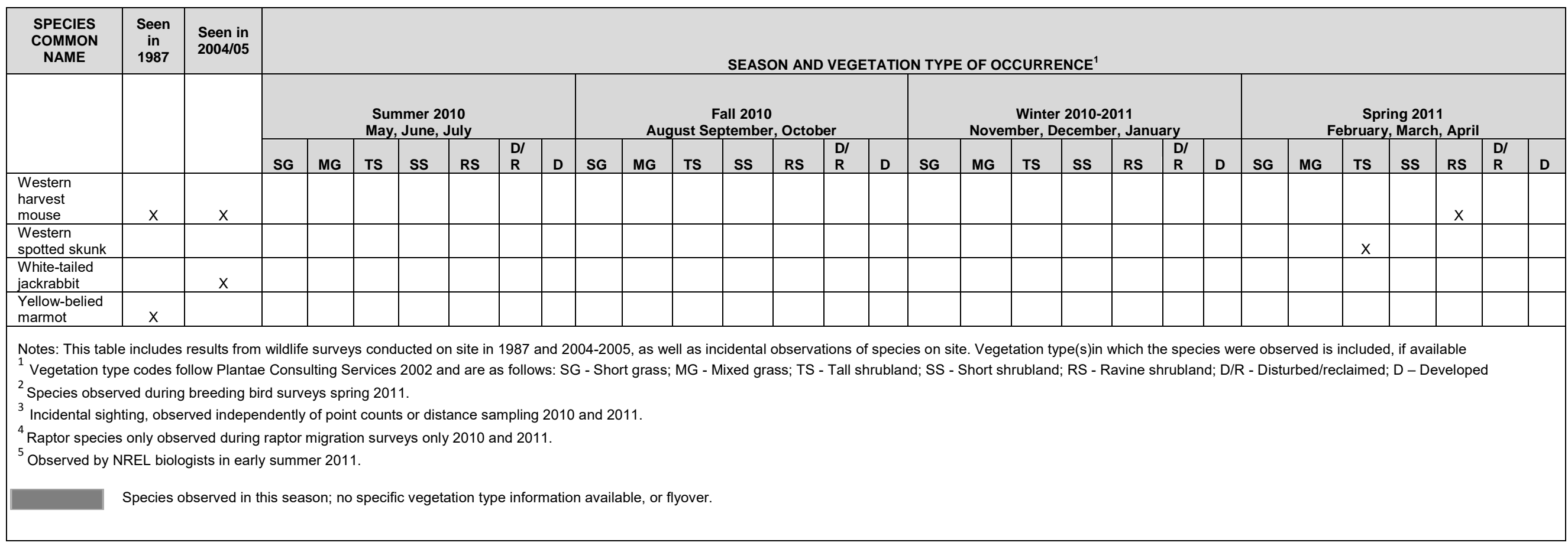




\section{General Wildlife Surveys}

$\underline{\text { Birds }}$

A total of 65 avian species were detected. The western meadowlark (Sturnella neglecta) was the most abundant species detected, and accounted for 12.5 percent of the total number of birds detected during avian point counts in 2010-2011. Rock pigeons (Columba livia) accounted for 11.8 percent, house finches (Carpodacus mexicanus) accounted for 10.9 percent, and black-billed magpies (Pica hudsonia) accounted for 9.2 percent of observations.

Avian species richness, diversity (dominance) and evenness are summarized in Table 4. In fall 2010 and spring and summer 2011, a similarly high richness of approximately 40 species was observed. Fourteen species were observed in winter. The total number of detected birds (n) was also similar in spring, summer and fall $(310,337$, and 338, respectively). The species diversity (dominance) was highest in the spring, summer, and fall (15.17, 14.95 and 12.35 , respectively) and lowest in winter (3.86).

Table 4. Avian Species Richness, Diversity (Dominance) and Evenness, Point Count Surveys, NREL South Table Mountain Site, 2010-2011

\begin{tabular}{|c|c|c|c|c|c|c|c|c|c|}
\hline \multicolumn{5}{|c|}{ SAIC Avian Point Count Data 2004-2005 } & \multicolumn{5}{|c|}{ Walsh Avian Point Count Data 2010-2011 } \\
\hline Season & $\begin{array}{c}\text { Species } \\
\text { Richness }^{1}\end{array}$ & $\mathbf{n}$ & $\begin{array}{c}\text { Species } \\
\text { Diversity }^{2} \\
\text { (Dominance) }\end{array}$ & $\begin{array}{c}\text { Species } \\
\text { Evenness }^{3}\end{array}$ & Season & $\begin{array}{c}\text { Species } \\
\text { Richness }^{1}\end{array}$ & $\mathbf{n}$ & $\begin{array}{c}\text { Species } \\
\text { Diversity }^{2} \\
\text { (Dominance) }\end{array}$ & $\begin{array}{c}\text { Species } \\
\text { Evenness }^{3}\end{array}$ \\
\hline Spring 2004 & 20 & 164 & 6.5 & 0.33 & $\begin{array}{c}\text { Spring } \\
2011\end{array}$ & 40 & 310 & 15.17 & 0.38 \\
\hline Summer 2004 & 20 & 193 & 7.6 & 0.39 & $\begin{array}{c}\text { Summer } \\
2010\end{array}$ & 41 & 337 & 14.95 & 0.36 \\
\hline Fall 2004 & 23 & 121 & 8.5 & 0.37 & Fall 2010 & 37 & 338 & 12.35 & 0.33 \\
\hline $\begin{array}{c}\text { Winter } \\
\text { 2004-2005 }\end{array}$ & 13 & 58 & 6.1 & 0.47 & $\begin{array}{l}\text { Winter } \\
2010- \\
2011\end{array}$ & 14 & 191 & 3.86 & 0.28 \\
\hline \multicolumn{10}{|c|}{$\begin{array}{l}{ }^{1} \text { Species richness: the number of species observed. } \\
2 \text { Species diversity (dominance): a measurement of dominance which assesses the probability that two randomly selected individuals } \\
\text { from a community will belong to the same species. } \\
{ }^{3} \text { Species evenness: a measure of the relative abundance of the different species making up the richness of an area. }\end{array}$} \\
\hline
\end{tabular}




\section{$\underline{\text { Raptors }}$}

A total of 29 individual raptors, comprising 10 species, were observed during the fall 2010 migration surveys (Table 5). Of the 29 individuals, 12 exhibited migratory behavior and 17 were considered local raptors. During the spring 2011 surveys a total of 15 individuals, comprising seven species, were observed. Nine individuals exhibited migratory behavior and six exhibited local behavior. The passage rate for migrant raptors was 1.50 and 1.13 raptors per hour for fall and spring, respectively, with an overall mean passage rate for both seasons combined of 1.31 raptors per hour.

No nesting raptors were found within the boundaries of STM during spring 2011 surveys.

Table 5. Raptor Survey Results, NREL South Table Mountain Site, 2010 and 2011

\begin{tabular}{|c|c|c|c|}
\hline Species & $\begin{array}{c}\text { Fall } 2010 \\
\text { Total (local)* }\end{array}$ & $\begin{array}{l}\text { Spring } 2011 \\
\text { Total (local)* }\end{array}$ & Total (local)* \\
\hline $\begin{array}{l}\text { Turkey vulture } \\
\text { (Cathartes aura) }\end{array}$ & $3(0)$ & - & $3(0)$ \\
\hline Osprey (Pandion haliaetus) & - & $1(1)$ & $1(1)$ \\
\hline $\begin{array}{l}\text { Sharp-shinned hawk (Accipiter } \\
\text { striatus) }\end{array}$ & $1(1)$ & $3(0)$ & $4(1)$ \\
\hline $\begin{array}{l}\text { Cooper's hawk } \\
\text { (Accipiter cooperii) }\end{array}$ & $3(1)$ & $4(0)$ & $7(1)$ \\
\hline $\begin{array}{l}\text { Northern goshawk (Accipiter } \\
\text { gentilis) }\end{array}$ & $1(0)$ & - & $1(0)$ \\
\hline $\begin{array}{l}\text { Broad-winged hawk (Buteo } \\
\text { platypterus) }\end{array}$ & - & $1(0)$ & $1(0)$ \\
\hline $\begin{array}{l}\text { Swainson's hawk (Buteo } \\
\text { swainsoni) }\end{array}$ & $1(1)$ & - & $1(1)$ \\
\hline $\begin{array}{l}\text { Red-tailed hawk (Buteo } \\
\text { jamaicensis) }\end{array}$ & $5(5)$ & $3(3)$ & $8(8)$ \\
\hline Buteo species (Buteo sp.) & $1(0)$ & - & $1(0)$ \\
\hline $\begin{array}{l}\text { American Kestrel (Falco } \\
\text { sparverius) }\end{array}$ & $12(7)$ & $2(1)$ & $14(8)$ \\
\hline $\begin{array}{l}\text { Peregrine falcon (Falco } \\
\text { peregrinus) }\end{array}$ & $1(1)$ & - & $1(1)$ \\
\hline Prairie falcon (Falco mexicanus) & $1(1)$ & - & $1(1)$ \\
\hline Falcon species (Falco sp.) & - & $1(1)$ & $1(1)$ \\
\hline
\end{tabular}


Table 5. Raptor Survey Results, NREL South Table Mountain Site, 2010 and 2011

\begin{tabular}{|l|c|c|c|}
\hline \multicolumn{1}{|c|}{ Species } & $\begin{array}{c}\text { Fall 2010 } \\
\text { Total (local)* }\end{array}$ & $\begin{array}{c}\text { Spring 2011 } \\
\text { Total (local)* }\end{array}$ & Total (local)* \\
\hline Migrant Raptor Total & 12 & 9 & 21 \\
\hline (Local Raptor Total) & $(17)$ & $(6)$ & $(23)$ \\
\hline $\begin{array}{l}\text { Total Raptors - Migrant and } \\
\text { Local }\end{array}$ & 29 & 15 & 44 \\
\hline $\begin{array}{l}\text { Migrant Passage Rate (raptors } \\
\text { per hour) }\end{array}$ & 1.50 & 1.13 & 1.31 \\
\hline
\end{tabular}

*Values in parentheses () indicate the subset of total observations that were non-migratory local raptors.

\section{$\underline{\text { Large Mammals }}$}

Results of the large mammal pellet plot counts is summarized by vegetation community in Figure 5. It should be noted that of the 76 pellet plots established 10 plots were vandalized and stakes removed within the short-grass vegetation type on top of the mesa during the spring 2011 survey. In addition, due to the expansion development at NREL, two plots were destroyed from construction activities.

\section{Figure 5. Number of Pellet Groups Observed per Vegetation Type, Large Mammal Surveys, NREL South Table Mountain Site, 2010-2011}

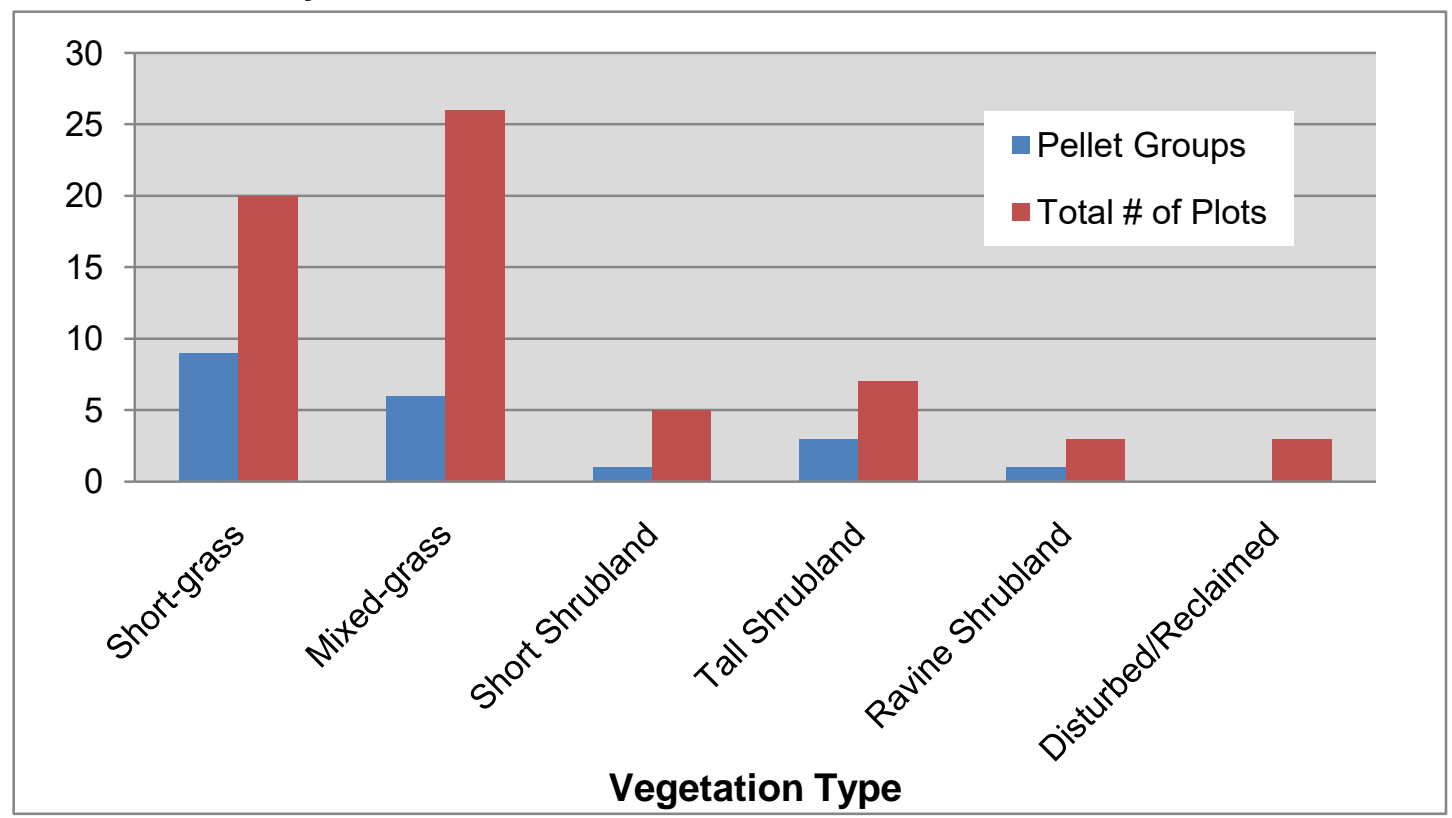

The short-grass and mixed-grass grassland vegetation had the highest number of pellets with nine and six groups, respectively. The tall shrubland had three and both the short shrubland and ravine shrubland had one pellet group each. 


\section{Mammalian Predators}

A total of 40 tracks were detected during the predator surveys (Table 6). These include four species of predators: coyote (Canis latrans), red fox (Vulpes vulpes), striped skunk (Mephitis mephitis), and western spotted skunk (Spilogale gracilis). Of the four species, coyote was detected the most with 20 tracks, followed by red fox with 14 tracks, and skunk with four tracks. Two tracks were unidentified. Predator activity was primarily concentrated in the short-grass vegetation on the mesa top with 27 tracks throughout the seasons while the mesa toe had 13 tracks. The winter months had highest activity with 20 different predator tracks detected.

Of the four skunk tracks, one set was noticeably smaller. The three larger tracks are undoubtedly the striped skunk. The third track, observed in April 2011, is attributed to the western spotted skunk.

\section{Table 6. Mammalian Predator Survey Results, NREL South Table Mountain} Site, 2010 and 2011

\begin{tabular}{|l|c|c|c|c|c|}
\hline \multicolumn{1}{|c|}{ Species } & $\begin{array}{c}\text { Summer } \\
\mathbf{2 0 1 0}\end{array}$ & Fall 2010 & $\begin{array}{c}\text { Winter } \\
\mathbf{2 0 1 0} \\
\mathbf{2 0 1 1}\end{array}$ & Spring 2011 & $\begin{array}{c}\text { Total Tracks } \\
\text { Observed }\end{array}$ \\
\hline Coyote (Canis latrans) & 1 & 4 & 10 & 5 & $\mathbf{2 0}$ \\
\hline $\begin{array}{l}\text { Red fox (Vulpes } \\
\text { vulpes) }\end{array}$ & 2 & 4 & 5 & 3 & $\mathbf{1 4}$ \\
\hline $\begin{array}{l}\text { Striped skunk } \\
\text { (Mephitis mephitis) }\end{array}$ & & & 3 & 1 & $\mathbf{3}$ \\
\hline $\begin{array}{l}\text { Western spotted } \\
\text { skunk (Spilogale } \\
\text { gracilis) }\end{array}$ & & & 2 & $\mathbf{2}$ & $\mathbf{2}$ \\
\hline Unidentified & $\mathbf{3}$ & $\mathbf{8}$ & $\mathbf{2 0}$ & $\mathbf{4 0}$ \\
\hline Total & & & & & \\
\hline
\end{tabular}

\section{$\underline{\text { Small Mammals }}$}

Small mammal trapping results are summarized in Table 7. In July 2010, a total of 32 individual small mammals were captured, with seven recaptures over three survey nights. All captures were of Mexican woodrats (Neotoma mexicana), with the exception of one deer mouse (Peromyscus maniculatus). The woodrats were captured in ravine shrublands on Transects DA and DC (Figure 1), and the deer mouse was captured at the top of Transect DC.

In April 2011, a total of 40 individual small mammals were captured, representing three species (Table 7). There were nine recaptures. Mexican woodrats and deer mice were captured in roughly equal number, and 11 western harvest mice (Reithrodontomys megalotis) were captured in ravine shrubland in Transects DA and DB (Figure 1).

The combination of both years reveals a species richness of three: deer mouse, western harvest mouse, and Mexican woodrat. Capture rates were slightly higher in April 2011 than in July 2010. 
In 2010, with 450 trap nights and a total of 39 captures (32 individuals and 7 recaptures), there was a 9 percent capture rate. In 2011, there was a total of 49 captures (40 individual captures and 9 recaptures), representing an 11 percent capture rate, and for the two seasons combined, was an overall capture rate of 10 percent.

Table 7. Small Mammal Captures, NREL South Table Mountain Site, 2010 and 2011

\begin{tabular}{|c|c|c|c|c|}
\hline Species & $\begin{array}{c}\text { Ravine } \\
\text { Shrubland }\end{array}$ & $\begin{array}{l}\text { Short-grass } \\
\text { Grassland }\end{array}$ & $\begin{array}{c}\text { Mixed-grass } \\
\text { Grassland }\end{array}$ & Total \\
\hline \multicolumn{5}{|l|}{ Summer 2010 (July 12 15) } \\
\hline $\begin{array}{l}\text { Deer Mouse } \\
\text { (Peromyscus maniculatus) }\end{array}$ & 1 & 0 & 0 & 1 \\
\hline $\begin{array}{l}\text { Mexican Woodrat } \\
\text { (Neotoma mexicana) }\end{array}$ & $30(7)$ & 1 & 0 & $31(7)$ \\
\hline \multicolumn{3}{|l|}{ Total } & & $32(7)$ \\
\hline \multicolumn{5}{|l|}{ Spring 2011 (April 25 28) } \\
\hline $\begin{array}{l}\text { Western Harvest Mouse } \\
\text { (Reithrodontomys } \\
\text { megalotis) }\end{array}$ & $11(1)$ & 0 & 0 & $11(1)$ \\
\hline $\begin{array}{l}\text { Deer Mouse } \\
\text { (Peromyscus maniculatus) }\end{array}$ & 7 & $6(3)$ & 0 & $13(3)$ \\
\hline $\begin{array}{l}\text { Mexican Woodrat } \\
\text { (Neotoma mexicana) }\end{array}$ & $16(5)$ & 0 & 0 & $16(5)$ \\
\hline \multicolumn{4}{|l|}{ Total } & 40(9) \\
\hline \multicolumn{5}{|l|}{2010 and 2011 Combined } \\
\hline \multicolumn{4}{|l|}{ Total } & $72(16)$ \\
\hline
\end{tabular}

Values based on 450 trap-nights per season, or 900 trap-nights for both years combined. There were 3 closed traps in 2010 and 10 closed traps in 2011. Values in parentheses ( ) indicate recaptures.

\section{$\underline{\text { Reptiles and Amphibians }}$}

Only three species of reptiles were found: western rattlesnake (Crotalus viridis), racer (Coluber constrictor), and prairie lizard (Sceloporus sp.). Woodhouse's toad (Bufo woodhousii) and tiger salamander (Ambystoma tigrinum) were the only amphibians noted. The Woodhouse's toad was detected during the nocturnal amphibian surveys discussed below in the Nocturnal Wildlife section. All reptiles and amphibians detections occurred within the summer season, with the exception of the tiger salamander, which was noted by NREL staff in 2011.

\section{Terrestrial Arthropods}

No terrestrial arthropod genera of specific concern were detected during surveys in June 2010. 


\section{Targeted Wildlife Surveys}

\section{Breeding Bird Densities}

A total of 749 individual detections and 450 clusters from 41 different bird species were observed along the eight transects. Eight of the 41 species were defined as flyovers. To remain consistent with distance sampling methods, these flyover data were not analyzed. Density was not analyzed for 28 other avian species due to their sample size (n) being too small $(\mathrm{n}<20)$. Buckland et al. (2001) suggest that a minimum sample size of 60 detections is needed for a reliable estimate of the detection function. Walsh was able to calculate densities for five avian species (Table 8). All species detected during distance sampling are summarized in Table 3.

The avian species with the highest density was by far Western meadowlark, with a density of 0.29 birds per hectare (Table 8). Although European starling (Sturnus vulgaris) had the second highest density of 0.22 birds per hectare, the co-efficient of variation of 0.22 was very high, causing the density measure to be unreliable and biased upwards.

Table 8. Breeding Bird Density Estimates, NREL South Table Mountain Site, 2011

\begin{tabular}{|c|c|c|c|c|c|c|}
\hline Species & $\begin{array}{c}\text { Sample } \\
\text { size } \\
\text { (clusters) }\end{array}$ & $\begin{array}{c}\text { Number of } \\
\text { Individuals }\end{array}$ & $\begin{array}{c}\text { Density } \\
\text { CV }\end{array}$ & $\begin{array}{c}\text { Density } \\
\text { Estimate } \\
\text { (Birds/hectare) }\end{array}$ & $\begin{array}{c}\text { Density } \\
95 \% \\
\text { Lower } \\
\text { Cl }\end{array}$ & $\begin{array}{c}\text { Density } \\
95 \% \\
\text { Upper } \\
\text { Cl }\end{array}$ \\
\hline $\begin{array}{c}\text { Western } \\
\text { Meadowlark } \\
\text { (Sturnella } \\
\text { neglecta) }\end{array}$ & 112 & 129 & 0.12 & 0.29 & 0.87 & 1.40 \\
\hline $\begin{array}{c}\text { European Starling } \\
\text { (Sturnus vulgaris) }\end{array}$ & 25 & 49 & 0.22 & 0.22 & 0.13 & 0.28 \\
\hline $\begin{array}{c}\text { Spotted Towhee } \\
\text { (Pipilo maculatus) }\end{array}$ & 51 & 52 & 0.13 & 0.20 & 0.10 & 0.39 \\
\hline $\begin{array}{c}\text { American Robin } \\
\text { (Turdus } \\
\text { migratorius) }\end{array}$ & 40 & 48 & 0.20 & 0.15 & 0.47 & 1.03 \\
\hline $\begin{array}{c}\text { Black-billed } \\
\text { Magpie } \\
\text { (Pica pica) }\end{array}$ & 34 & 52 & 0.21 & 0.08 & 0.06 & 0.11 \\
\hline
\end{tabular}

\section{Nocturnal Wildlife}

Nocturnal playback surveys for eastern screech-owl failed to detect or elicit any responses during the March 2011 surveys. No other owl species were detected during nocturnal surveys for other genera. Great horned owls are known to occur in the area, although no nests have been found, they have been observed hunting (Brenda Beatty - NREL, pers. com. 2011). 
During the nocturnal amphibian surveys, Woodhouse's toad was detected in the ephemeral ponds on the mesa top northeast of the Solar Industrial Mesa Test Area. The Wisconsin index value assigned to each observation was $2=$ calls are overlapping, but individuals are still distinguishable.

No bat species were detected during the acoustical bat surveys conducted in the summer of 2010 .

\section{$\underline{\text { Mule Deer }}$}

During the summer 2010 mule deer survey, four bucks, ten does, and two fawns were located (Figure 6). The mule deer concentrated in two groups with one group located on the southwestern facing hillside of the east drainage and the other located in the amphitheater.

In winter of 2010, three bucks, eleven does, and five fawns were found in the east drainage.

\section{$\underline{\text { Carnivore Camera }}$}

Throughout the four seasons, the Trailmaster monitors with connected cameras took three carnivore photographs, all of one predator species, the coyote. Coyotes were detected in the east drainage in the summer and winter of 2010 and one coyote was detected in the amphitheater in the spring of 2011. In addition, other detections included numerous mule deer, one cottontail (Sylvilagus sp.), one Mexican woodrat, and two black-billed magpies.

\section{Special Status Species}

A peregrine falcon (Falco peregrinus), which is a State Species of Special Concern, was observed. No other wildlife Special Status Species were observed during any surveys. 


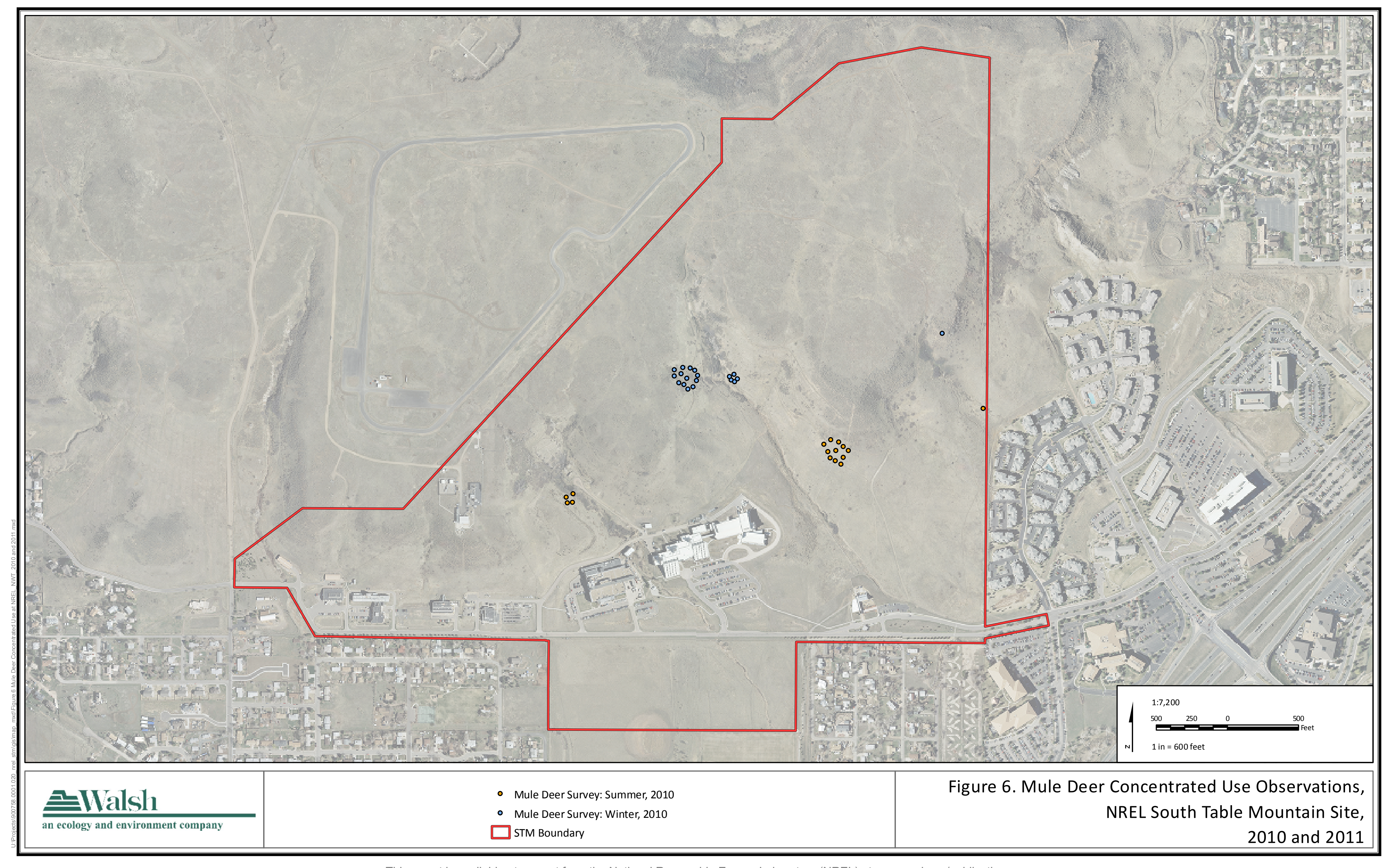

This report is available at no cost from the National Renewable Energy Laboratory (NREL) at www.nrel.gov/publications 


\section{DISCUSSION}

\section{Vegetation Mapping}

Few changes in the plant community boundaries were noted since these areas were previously mapped (Plantae 2002). Observed trends in vegetation patterns include a general increase in invasive and noxious weed species diversity and extent across the site. Some factors that may be contributing to this apparent trend include changes in land use, such as surface disturbing activities since 2002, and drought conditions.

\section{Grasslands}

The Plantae (2002) vegetation mapping effort characterized the majority of the mesa top on the STM site as short-grass grassland. The community supports a diverse mix of grass species, as it did in 2002, but percent cover of weeds, especially musk thistle, has increased. One possible reason for the increase in noxious weeds might be drought conditions occurring in many of the growing seasons since 2002 .

The current mapping effort found mixed-grass grassland as the dominant community in the central and southern portions of the site. Characteristics of this community appear similar to those observed in 2002. The noxious weed species that has increased the most throughout this community appears to be Canada thistle. Three small seeps that were not observed in 2002 were found in the southeastern portion of this plant community.

\section{Upland Shrublands}

Herbaceous layer characteristics, including species composition and areas lacking cover, in both shrubland communities, remains comparable to 2002 observations. The most notable change in the upland shrublands since 2002 is that musk thistle has colonized these areas.

\section{$\underline{\text { Ravine Shrublands }}$}

No significant changes to plant community boundaries were observed in 2010 as compared to 2002. No new noxious weed species were observed here since these areas were last mapped in 2002 .

\section{$\underline{\text { Wetlands }}$}

The areas of wetland vegetation have not been jurisdictionally delineated. Due to a dry winter in 2010 , followed by a dry spring in 2011 , borders of wetland communities were not able to be confirmed with assurance for this report.

The wetland community observed at the top of the southwest drainage in 2002 is still dominated by obligate and facultative wetland plants but the dominant species have changed from cattails to graminoids including sedges, rushes, and bulrush species. The peach-leaf willows observed in 2002 were also observed in 2010 . Changes in vegetation composition may be due to land use changes in nearby developed areas.

When visited in late June 2010, the linear depression wetland contained saturated soils and standing water. Field bindweed (Convolvulus arvensis) observed in this area in 2010, was not present in the wetland communities in 2002 (Plantae). Field bindweed may have established here as a result of surface disturbance due to construction activities adjacent to this wetland. 


\section{Disturbed/Reclaimed}

There are more disturbed/reclaimed communities within the STM boundaries than there were in 2002. Likely the increase in surface disturbing activities contributed to the establishment of whitetop, Canada thistle, yellow sweetclover (Melilotus officinalis), and common dandelion (Taraxicum officinale), all species observed for the first time in 2010. These species likely colonized areas of surface disturbance that resulted from construction activities.

\section{Noxious Weeds}

The STM site contains 12 plant species found on the State of Colorado Noxious Weed List (Table 1). Of these species, five are included in the top ten priority weeds for Colorado, as listed in the Colorado Weed Management Act. These species are the most widespread and cause the greatest economic impact to the state and should be considered a priority-for-control for the STM site.

Historically, the short-grass grassland found on the mesa top was probably dominated by blue grama grass and other short-grass species such as buffalo grass (Buchloë dactyloides). However, this entire mesa-top area has become dominated by cheatgrass, an aggressive noxious weed. This weed is changing the appearance and general species composition of the area by apparently outcompeting native plants.

The majority of noxious weed populations have remained unchanged since the site was last surveyed in 2002 by Plantae. However, some weed populations have expanded such as musk thistle, dalmatian toadflax, Canada thistle, and diffuse knapweed. In addition, these weed populations will likely spread in response to the disturbance of large swaths of mixed-grass grassland associated with the construction of additional NREL facilities in 2010 and 2011.

\section{Wildlife Survey Results}

During 2010/2011 a total of 102 species of wildlife were observed at STM. In comparison, the 1987 surveys (FORUM 1987) observed 31 species, and in 2004/2005 SAIC (SAIC 2005) observed 69 species (Table 3). In 1987, two reptile species, one bird species, and six mammal species were documented that were not seen in 2010/2011. In 2004/2005, two herptiles, 13 bird species, and six mammal species were documented for STM that were not observed in 2010/2011.

Surveys in 2010/2011 detected 77 species (four herptiles, 70 birds, and four mammals) not documented during surveys conducted in 1987. Forty-seven species were added to the site species list since surveys conducted in 2004/2005 (three herptiles, 41 birds, and three mammals).

The cause of these differences may be attributed to the relative level of effort between different surveys, respectively. They may also be related to differences in observer ability, changes in species composition that affect occurrence of other species, observation conditions during surveys, and/or differences in seasonal weather patterns and recent site development activities.

\section{General Wildlife Surveys}

\section{$\underline{\text { Birds }}$}

The seasonal average for avian species richness, diversity (dominance) and total number of detections all approximately doubled since 2004/2005. This drastic increase in the bird community may be due to factors such as change in observers, level of effort, weather patterns, or other causes not apparent.

The total number of birds detected during point counts in 2010/2011 was 1,216 (56 percent more birds detected when compared to 2004/2005). The species richness in 2010/2011 increased 
overall by 44 percent since 2004/2005. Avian diversity (dominance) also significantly increased by 38 percent since the 2004/2005. Evenness in spring, summer and fall of 2010/2011 remained similar to 2004/2005. Evenness in the winter 2010/2011 dropped from 47 in 2004/2005 to 28. In winter 2010/2011, rock pigeons made up 46 percent of the total detected birds, thus accounting for the decreased evenness.

The western meadowlark was the most abundant species in spring and summer and remained the most abundant bird overall throughout both studies. In fall the most abundant species was the house finch for all years. In the winter, rock pigeons were most abundant in 2010/2011, while in 2004/2005 the black-billed magpie was most abundant. Declines in population can be related to disease, changes in habitat, and shifts in food resources available to the species.

Although avian diversity (dominance) (Simpson index) was estimated to remain consistent with the SAIC report, NREL may prefer to use the modified Shannon index in the future rather than dominance. Buckland et al. (2005) investigated what features define a good diversity index. Although they conclude that no single index can capture all aspects of diversity change, the modified Shannon index performed better than the Simpson's index. Therefore Walsh recommends using the modified Shannon index in the future to more accurately monitor changes in biodiversity at NREL.

\section{$\underline{\text { Raptors }}$}

Raptor observations during the two seasons of surveys were characteristic of local and migratory species known to occur within the region. In comparison to the 2004/2005 surveys in which 10 species were observed (SAIC 2005), the 2010/2011 surveys detected four additional species for STM: turkey vulture (Cathartes aura), northern goshawk (Accipiter gentilis), broad-winged hawk (Buteo platypterus), and peregrine falcon (Falco peregrinus). In 1987 only two species were observed: red-tailed hawk (Buteo jamaicensis) and American kestrel (Falco sparverius) (FORUM 1987). One species, golden eagle (Aquila chrysaetos), was detected in the 2004/2005 raptor surveys and was not observed in the 2010/2011 surveys. Overall the 2010/2011 raptor surveys detected 11 species plus two additional species identified only to genera (Buteo sp., Falco sp.). One additional species, northern harrier (Circus cyaneus) was observed outside the raptor survey periods.

No nesting raptors were detected onsite. In comparison, 2004/2005 surveys detected nesting American kestrel, and 1987 surveys detected nesting red-tailed hawk and American kestrel. Although neither species were detected onsite, red-tailed hawks were known to be nesting just south of STM in Lena Gulch. The high number of non-migrant American kestrels (8) may be indicative of local nesting within habitat adjacent to STM.

\section{$\underline{\text { Large Mammals }}$}

There is difficulty in comparing these data with the earlier SAIC (2005) data for two reasons: the current study collected the data after three months of pellet accumulation from 66 plots, whereas the earlier study collected data after six months from 97 plots. However, the trends of use of the different vegetation communities are similar between the two studies: the greatest use was in short-grass grassland, followed by mixed-grass grassland, tall shrubland, and short shrubland and ravine shrubland. During this and other field efforts, it was apparent that the mule deer use the ravine shrublands for cover and resting sites. The high number of pellet groups in the short-grass may be due to the location of 20 plots in this community type (the second largest number of plots after mixed-grass), and this community represented the type with the least amount of human disturbance. The mixed-grass community type, which had the most plots, had fewer pellet groups probably because this type was closest to the NREL development. 


\section{Mammalian Predators}

Coyote and red fox are very common predators along the Front Range, and their presence suggests that there is good denning and foraging habitat on site. The presence of the western spotted skunk is of interest as the species is generally present but not commonly detected. They use the short-grass habitat and likely den in the rocky ravine areas. Tracks were observed at Station 2, on the eastern edge of the mesa (Figure 1).

The previous study found only coyotes. With the current study, three additional species have been detected: red fox, western spotted skunk, and striped skunk. The highest number of tracks was found during winter.

\section{$\underline{\text { Small Mammals }}$}

Although results were generally consistent between the July 2010 and April 2011 trapping surveys, densities of small mammals undergo annual fluctuations as is reflected in trapping response and capture rates (Smith et al. 2009). Deer mice are a ubiquitous species, sometimes referred to as a weedy species due to their ability to occur in virtually all habitats except wetlands (Armstrong et al. 2011). What is surprising is the lack of captures of deer mice in 2010, when only one mouse was captured during 450 trap nights; and that deer mice did not dominate the captures in both periods. The reason for this is not clear. Deer mice are susceptible to hantavirus, although there has been no known outbreak of the disease in 2010 or 2011 along the Front Range.

The species richness for three species of small mammals in 2010/2011 combined at STM is low for small mammals. Species richness was higher in 1987 and 2004/2005 when prairie voles were present (FORUM 1987) and in 2004/2005 (SAIC 2005). Prairie voles may have been

experiencing a population low during the 2010/2011 surveys. Although prairie voles in Colorado may not experience the population cycles typical of the genus elsewhere, 2 to 4 year cycles have been found in other studies (Armstrong 2011).

It is noteworthy that bushy-tailed woodrats (Neotoma cinerea) were found in 1987 (FORUM 1987), but Mexican woodrats were not found. These two species are sympatric along a narrow corridor of the Front Range (Finley 1958). It is not known if the Mexican woodrat displaced the bushy-tailed woodrat or if it was present in 1987 but not detected.

\section{$\underline{\text { Reptile and Amphibians }}$}

Western rattlesnakes, racers, prairie lizards, tiger salamanders, and Woodhouse's toads are all widespread and common in Colorado. Habitats for racer include prairie grasslands, sandhills, open riparian woodlands, and shrubby foothills and canyons. The western rattlesnake occurs in virtually every terrestrial habitat within Colorado (Hammerson 1999). Woodhouse's toad is the state's most commonly encountered amphibian and tiger salamanders occur in virtually any habitat, provided there is standing water such as permanent or ephemeral ponds (Hammerson 1999).

Western rattlesnakes were found in 1987 (FORUM 1987) and again in 2004/2005 (SAIC 2005). However, tiger salamanders were found only in 2004/2005 (SAIC 2005) and Woodhouse's toad, prairie lizards, and racers were all not detected during the previous surveys. The 1987, $2004 / 2005$, and 2010/2011 reptile and amphibian surveys were opportunistic and it is likely that most of these species were present but not detected.

\section{Terrestrial Arthropods}

Surveys for terrestrial arthropod genera did not detect any Species of Concern. Records from CNHP do not have any specific sightings from STM; most of the historic records (the most recent 
documented in 1991) are located north and west of STM (Appendix C). As most of the species depend on native grasses for larval food, further encroachment of noxious weed species limit the potential for any genera or species of concern to inhabit STM. The limited nature of these surveys should be noted.

\section{Targeted Wildlife Surveys}

\section{Breeding Bird Densities}

Spring 2011 was the first year distance sampling was performed on the NREL STM avian community to estimate breeding bird densities. There were limitations in estimating breeding songbird densities because of low sample sizes and only one season of data. Continued monitoring of avian species may contribute to a better density estimate and is recommended. With increased sampling over additional years, more comprehensive density estimates may be obtained.

\section{Nocturnal Wildlife}

Limited cottonwoods and pine stands on the STM site likely preclude the presence of eastern screech-owl. Even though the eastern screech-owl has the broadest ecological niche of any owl in its range (Gehlbach 1995), they generally prefer mature stands of cottonwoods (Steve Jones personal communication 2011).

Woodhouse's toads were the only amphibians heard on site and they were heard at the only two pools. The lack of other amphibians is not surprising due to the general scarcity of surface water on the STM site.

The absence of bat activity on the site was likely due to the limited sampling (one monitor for two nights) that was conducted in early June. This is not a time period of high bat activity. Typically, bats in Colorado are most active in July, August, and September. Some bats are still arriving at their summer roosts in June. A more comprehensive bat survey, including greater sampling effort and extension of the summer season, would surely be of use in documenting bat activity on the site.

\section{$\underline{\text { Mule Deer }}$}

Mule deer are found throughout the state in every ecosystem with the foothills of the Front Range having one of the highest densities (Armstrong et al. 2011). Large herds of mule deer were noted on the STM site, with the highest concentrations found throughout the seasons in the steep and broken terrain of the shrublands, which provide abundant browse and cover. In the winter months, the size of herds increases in general and large numbers may congregate on wintering grounds (Armstrong et al. 2011). This behavior was consistent with mule deer surveys and observational results found on the STM site. In the summer months, the mule deer were found in small groups with the largest herd containing 14 individuals. Whereas in the winter survey one large herd was found containing 18 deer and an additional observation in January 2011 recorded during other wildlife surveys, noted a herd containing 28 mule deer. This suggests that the STM site is likely used as a wintering ground which is consistent with higher use found in the winter in previous surveys (SAIC 2005). Winter habitat is typically a limiting resource for big game species such as mule deer.

\section{$\underline{\text { Carnivore Camera }}$}

Coyotes were the only predator detected in the mammalian predator surveys with scent stations in surveys done by SAIC in 2005 and the most common predator detected by Walsh in 2010/2011, 
so it is no surprise that coyotes were the only carnivore detected with the cameras as well.

Coyotes are found in every ecosystem throughout Colorado and are one of the most adaptable carnivores in North America (Armstrong et al. 2011).

A small pack of four coyotes were noted in spring of 2011 above the amphitheater by the solar facility. This pack may have been associated with the nearby den found in the rocky outcrop by SAIC in 2005. The abundant rodents, rabbits, and fawns found on STM provide an essential food resource. In addition, evidence of these coyotes working cooperatively on the predation of a full grown buck in the east drainage was discovered in March of 2011.

\section{Special Status Species}

One Special Status Species, the peregrine falcon, was observed during the year-long surveys. Observed during the fall raptor surveys in 2010, it was characterized as a local bird by its behavior. No longer considered endangered by state and federal agencies, it remains a Species of Special Concern (non-statutory) for Colorado due to its specialized breeding habitat (undisturbed cliffs) and low reproductive rates.

\section{RECOMMENDATIONS}

Based on the observations discussed above, there are several measures and recommendations that could minimize impacts to wildlife. These include:

- Develop the smallest possible footprint for buildings, access roads, and other infrastructure.

- Minimize disturbance to native plant communities to benefit native grassland species of amphibians, reptiles, birds, and mammals.

- To protect native plant community biodiversity, eradicate small weed populations, monitor for new weed infestations, and actively manage weeds onsite through NREL's Weed Management Program for the STM (6-2.12).

- Minimize the potential for weed invasions that replace the native grassland plants during construction and ongoing activities in the conservation zones such as recreational activities.

- Seek opportunities for creation of wildlife habitat corridors to connect to other nearby areas of habitat, especially to the open spaces to the north and west, and to Lena Gulch to the south.

- Protect the small hill seeps to the east of the Visitor Center from encroaching construction activities.

- Conduct additional bat surveys, ensuring coverage of July, August, and September, as well as multiple locations on site.

- Continued monitoring of avian species will contribute to more robust density estimates and such surveys should be conducted at intervals shorter than five years. 


\section{REFERENCES}

Animal Care and Use Committee. 1998. Guidelines for the capture, handling, and care of mammals as approved by the American Society of Mammalogists. Journal of Mammalogy 79:1416-1431.

Armstrong, D.M., J. P. Fitzgerald, and C. A. Meaney. 2011. Mammals of Colorado, Second Edition. University Press of Colorado, Niwot. 620 pp.

Begon, M., C.R. Townsend, and J.L. Harper. 2006. Ecology: from individuals to ecosystems. Blackwell Publishing. Fourth Edition.

Buckland, S. T., Anderson, D. R., Burnham, K. P., and Laake, J. L. 1993. Distance Sampling: Estimating Abundance of Biological Populations. London: Chapman and Hall.

Buckland, S. T., Anderson, D. R., Burnham, K. P., Laake, J. L., Borchers, D. L., and Thomas, L. 2001. Introduction to Distance Sampling. Oxford: Oxford University Press

Buckland, S.T., A.E Magurran, R.E Green, and R.M Fewster. 2005. Monitoring change in biodiversity through composite indices. Phil Trans R Soc B, 360: 243-254.

Colorado Department of Agriculture (CDOA). 2010. Colorado Noxious Weed List. http://www.colorado.gov/cs/Satellite/Agriculture-Main/CDAG/1174084048733. Accessed online 6/2010.

Colorado Division of Wildlife (CDOW). 2010. Species of Concern. http://wildlife.state.co.us/WildlifeSpecies/SpeciesOfConcern/ThreatenedEndangeredList/ ListOfThreatenedAndEndangeredSpecies.htm

Droege, S. Amphibian Calling Surveys. USGS Patuxent Wildlife Research Center, Laurel, MD. http://www.pwrc.usgs.gov/monmanual/techniques/amphibcallingsurveys.htm. Accessed online $3 / 2010$.

Finley, R.B., Jr. 1958. The Woodrats of Colorado: distribution and ecology. University of Kansas Publications, Museum of Natural History, 10(6):213-552.

Francis, C.M. and Bradstreet, M.S.W. 1997. Monitoring Boreal Forest Owls in Ontario using tape playback surveys with volunteers. In: Duncan, James R.; Johnson, David H.; Nicholls, Thomas H., eds. Biology and conservation of owls of the Northern Hemisphere: 2nd International symposium. Gen. Tech. Rep. NC-190. St. Paul, MN: U.S. Dept. of Agriculture, Forest Service, North Central Forest Experiment Station. 175-184.

Gehlbach, F. R. 1995. Eastern Screech-Owl (Megascops asio), The Birds of North America Online (A. Poole, Ed.). Ithaca: Cornell Lab of Ornithology; Retrieved from the Birds of North America Online: http://bna.birds.cornell.edu/bna/species/165

Hammerson, G.A. 1999. Amphibians and Reptiles in Colorado: A Colorado Field Guide. Second Edition. University Press of Colorado and Colorado Division of Wildlife, Colorado.

Hawk Migration Association of North America (HMANA). 2010. Daily reporting form instructions. http://www.hmana.org/documents/drf2.xls. Accessed online 3/2010.

Linhart, S.B. and F.F. Knowlton. 1975. Determining the Relative Abundance of Coyotes by Scent Station Lines. Wildlife Society Bulletin 3(3):119-124.

McShea, W.J. 2008 Butterfly Mapping Project. Smithsonian Conservation and Research Center. Front Royal, Virginia. 
Mills, J.N., T.L. Yates, J.E. Childs, R.R. Parmenter, T.G. Ksiazek, P.E. Rollin, and C.J. Peters. 1995. Guidelines for working with rodents potentially infected with Hantavirus. Journal of Mammalogy, 76(3):716-722.

National Renewable Energy Laboratory (NREL). No Date. EHS Program Number 6-2.12, Weed Management Program, STM Site (Draft). Golden, Colorado.

Neff, D.J. 1968. The Pellet-Group Count Technique for Big Game Trend, Census, and Distribution: A Review. Journal of Wildlife Management 32(3):597-614.

Pearson and Ruggiero. 2003. Transect versus grid trapping arrangements for sampling smallmammal communities. Wildlife Society Bulletin 31(2):597-614.

Plantae Consulting Services. 2002. Vegetation Survey Report. National Renewable Energy Laboratory South Table Mountain Site. June 29, 2002.

Science Applications International Corporation (SAIC). 2005. Wildlife Survey (Including Migratory Birds and Raptors) at the National Renewable Energy Laboratory South Table Mountain Site Golden, Colorado. June 22, 2005.

Sikes, R.S., W.L. Gannon, and the Animal Care and Use Committee of the American Society of Mammalogists. 2011. Guidelines of the American Society of Mammalogists for the use of wild mammals in research. Journal of Mammalogy, 92(1):235-253.

Simpson, E.H. 1949. Meaurement of Diversity. Nature. Macmillian Publishers Ltd. 163: 688.

Smith, M.H. et al. 2009. Density estimations of small mammal populations. Pp 25-53 in (F.B. Golley, K. Petrusewicz, and L. Ryszkowski, eds.) Small mammals: their productivity and population dynamics. Cambridge University Press, Cambridge.

The FORUM Associates, Inc. (FORUM) 1987. Solar Energy Research Institute Wildlife Report. September 1987.

Thomas, L., S.T. Buckland, E.A. Rexstad, J. L. Laake, S. Strindberg, S. L. Hedley, J. R.B. Bishop, T. A. Marques, and K. P. Burnham. 2010. Distance software: design and analysis of distance sampling surveys for estimating population size. Journal of Applied Ecology 47: 5-14.

U.S. Fish and Wildlife Service (USFWS). July 2010. Endangered, Threatened, and Candidate Species and Designated Critical Habitat for Colorado Counties. http://www.fws.gov/mountain\%2Dprairie/endspp/countylists/colorado.pdf

Van Dyke, F. 2008. Conservation biology: foundations, concepts, applications. Springer, Second Edition.

http://books.google.com/books?id=Evh1UD3ZYWcC\&pg=PA91\&dq=species + diversity + index + and + dominance \&hl=en\&ei=hOFCTu3MJcTcgQfjg7y2CQ\&sa $=X \&$ oi=book res ult\&ct $=$ bookthumbnail\&resnum $=7 \& v e d=0 \mathrm{CE} 0 \mathrm{Q} 6 \mathrm{wEwBg} \# \mathrm{v}=$ onepage $\& \mathrm{q}=$ species $\% 20 \mathrm{di}$ versity $\% 20$ index $\% 20$ and $\% 20$ dominance $\& f=$ false

Weber, W.A. and R.C. Wittmann. 2001. Colorado Flora: Eastern Slope. University Press of Colorado. Third Edition. 


\section{APPENDIX A \\ Plant Community Species List}




\section{Legend to classification codes:}

\section{Origin:}

Refers to origin of species

$\mathrm{N} \quad$ Native to the Front Range area

I Introduced or exotic species

\section{Species:}

Refers to blooming/production season for grass species

C cold season (spring/early summer)

W warm season (mid to late summer)

\section{Life Form:}

Refers to life form/strategy of species

AF annual forb

BF biennial forb

PF perennial forb

AG annual grass/graminoid (includes rushes and sedges)

PG perennial grass/graminoid (includes rushes and sedges)

SU succulent

SS subshrub

S shrub

$T$ tree

V vine 
Table 1. Grassland Plant Community

\begin{tabular}{|c|c|c|c|c|c|c|}
\hline \multicolumn{7}{|c|}{$\begin{array}{l}\text { Plant Species List } \\
\text { NREL South Table Mountain Site }\end{array}$} \\
\hline Scientific Binomial & Synonomy & Common Name & Family & Origin & Season & $\begin{array}{l}\text { Life } \\
\text { Form }\end{array}$ \\
\hline Adenolinum lewisii & Linum lewisii & Wild flax & Linaceae - Flax Family & $\mathrm{N}$ & NA & PF \\
\hline Aegilops cylindrica & & Jointed goatgrass & Poaceae - Grass Family & I & C & $A G$ \\
\hline Agaloma marginata & & Snow-on-the-mountain & Euphorbiaceae - Spurge Family & $\mathrm{N}$ & NA & PF \\
\hline Agoseris glauca & & False dandelion & Asteraceae - Sunflower Family & $\mathrm{N}$ & NA & PF \\
\hline Agropyron cristatum & & Crested wheatgrass & Poaceae - Grass Family & I & C & $P G$ \\
\hline Allium sp. & & Wild onion & Alliaceae - Onion Family & $\mathrm{N}$ & NA & PF \\
\hline Alyssum parviflorum & Alyssum minus & Alyssum & Brassicaceae - Mustard Family & 1 & NA & AF \\
\hline Ambrosia psilostachya & & Western ragweed & Asteraceae - Sunflower Family & $\mathrm{N}$ & NA & PF \\
\hline Andropogon gerardii & & Big bluestem & Poaceae - Grass Family & $\mathrm{N}$ & W & PG \\
\hline Anisantha tectorum & Bromus tectorum & Cheatgrass & Poaceae - Grass Family & I & C & $A G$ \\
\hline Apocynum cannabinum & & Indian hemp & Apocynaceae - Dogbane Family & $\mathrm{N}$ & NA & PF \\
\hline Argemone hispida & & Hairy poppy & Papaveraceae - Poppy Family & $\mathrm{N}$ & NA & PF \\
\hline Aristida purpurea & & Three-awn & Poaceae - Grass Family & $\mathrm{N}$ & W & PG \\
\hline Artemisia biennis & & Sagewort & Asteraceae - Sunflower Family & I & NA & PF \\
\hline Artemisia frigida & & Fringed sagebrush & Asteraceae - Sunflower Family & $\mathrm{N}$ & NA & SS \\
\hline Artemisia ludoviciana & & Prairie sagewort & Asteraceae - Sunflower Family & $\mathrm{N}$ & NA & PF \\
\hline Asclepias pumila & & Plains milkweed & Aclepiadaceae - Milkweed Family & $\mathrm{N}$ & NA & PF \\
\hline
\end{tabular}


Table 1. Grassland Plant Community

\begin{tabular}{|c|c|c|c|c|c|c|}
\hline \multicolumn{7}{|c|}{$\begin{array}{c}\text { Plant Species List } \\
\text { NREL South Table Mountain Site }\end{array}$} \\
\hline Scientific Binomial & Synonomy & Common Name & Family & Origin & Season & $\begin{array}{l}\text { Life } \\
\text { Form }\end{array}$ \\
\hline Asclepias speciosa & & Showy milkweed & Asclepiadaceae - Milkweed Family & $\mathrm{N}$ & NA & PF \\
\hline Aster porteri & & White aster & Asteraceae - Sunflower Family & $\mathrm{N}$ & NA & PF \\
\hline Astragalus drummondii & & Drummonds milk vetch & Fabaceae - Pea Family & $\mathrm{N}$ & NA & PF \\
\hline Astragalus shortianus & & Milk vetch & Fabaceae - Pea Family & $\mathrm{N}$ & NA & PF \\
\hline Bassia sieversiana & Kochia scoparia & Kochia & $\begin{array}{l}\text { Chenopodiaceae - Goosefoot } \\
\text { Family }\end{array}$ & I & NA & AF \\
\hline Bouteloua curtipendula & & Side-oats grama & Poaceae - Grass Family & $\mathrm{N}$ & W & $P G$ \\
\hline $\begin{array}{l}\text { Brickellia rosmarinifolia } \\
\text { subsp. chlorolepis }\end{array}$ & Kuhnia chlorolepis & Brickellia & Asteraceae - Sunflower Family & $\mathrm{N}$ & NA & PF \\
\hline Bromopsis inermis & Bromus inermis & Smooth bromegrass & Poaceae - Grass Family & 1 & C & $P G$ \\
\hline Bromus japonicus & & Japanese brome & Poaceae - Grass Family & 1 & C & $A G$ \\
\hline Carduus nutans & & Musk thistle & Asteraceae - Sunflower Family & I & NA & $\mathrm{BF}$ \\
\hline Carex brevior & & Sedge & Cyperaceae - Sedge Family & $\mathrm{N}$ & NA & PG \\
\hline $\begin{array}{l}\text { Carex pensylvanica subsp. } \\
\text { heliophila }\end{array}$ & & Sun sedge & Cyperaceae - Sedge Family & $\mathrm{N}$ & NA & PG \\
\hline Castilleja integra & & Indian paintbrush & Scrophulariaceae - Figwort Family & $\mathrm{N}$ & NA & PF \\
\hline Celtis reticulata & & Netleaf hackberry & Ulmaceae - Elm Family & $\mathrm{N}$ & NA & $\mathrm{T}$ \\
\hline Acosta diffusa & Centaurea diffusa & Diffuse knapweed & Asteraceae - Sunflower Family & I & NA & $\mathrm{BF} / \mathrm{PF}$ \\
\hline $\begin{array}{l}\text { Chamaesyce } \\
\text { glyptopsperma }\end{array}$ & $\begin{array}{l}\text { Euphorbia } \\
\text { glyptosperma }\end{array}$ & Ridgeseed spurge & Euphorbiaceae - Spurge Family & $\mathrm{N}$ & NA & AF \\
\hline Chenopodium berlandieri & & Goosefoot & $\begin{array}{l}\text { Chenopodiaceae - Goosefoot } \\
\text { Family }\end{array}$ & $\mathrm{N}$ & NA & AF \\
\hline
\end{tabular}


Table 1. Grassland Plant Community

\begin{tabular}{|c|c|c|c|c|c|c|}
\hline \multicolumn{7}{|c|}{$\begin{array}{c}\text { Plant Species List } \\
\text { NREL South Table Mountain Site }\end{array}$} \\
\hline Scientific Binomial & Synonomy & Common Name & Family & Origin & Season & $\begin{array}{l}\text { Life } \\
\text { Form }\end{array}$ \\
\hline Chondrosum gracile & Bouteloua gracilis & Blue grama & Poaceae - Grass Family & $\mathrm{N}$ & W & $P G$ \\
\hline $\begin{array}{l}\text { Chrysothamnus nauseosus } \\
\text { subsp. graveolens }\end{array}$ & & Rubber rabbitbrush & Asteraceae - Sunflower Family & $\mathrm{N}$ & NA & $\mathrm{S}$ \\
\hline Breea arvensis & Cirsium arvense & Canada thistle & Asteraceae - Sunflower Family & I & NA & PF \\
\hline Cirsium canescens & & Hairy thistle & Asteraceae - Sunflower Family & $\mathrm{N}$ & NA & PF \\
\hline Cirsium undulatum & & Wavyleaf thistle & Asteraceae - Sunflower Family & $\mathrm{N}$ & NA & $\mathrm{BF}$ \\
\hline Commandra umbellata & & Bastard-toadflax & Santalaceae - Sandelwood Family & $\mathrm{N}$ & NA & PF \\
\hline Convolvulus arvensis & & Field bindweed & Convolvulaceae - Morning Glory & 1 & NA & PF \\
\hline Coreopsis tinctoria & & Plains coreopsis & Family & $\mathrm{N}$ & NA & PF \\
\hline Coryphantha missouriensis & & Yellow pincushion & Cactaceae - Cactus Family & $\mathrm{N}$ & NA & SU \\
\hline $\begin{array}{l}\text { Coryphantha vivipara var. } \\
\text { vivipara }\end{array}$ & & Nipple cactus & Cactaceae - Cactus Family & $\mathrm{N}$ & NA & SU \\
\hline Critesion jubatum & Hordeum jubatum & Foxtail barley & Poaceae - Grass Family & $\mathrm{N}$ & C & PG \\
\hline Cynoglossum officinale & & Houndstongue & Boraginaceae - Borage Family & 1 & NA & $\mathrm{BF}$ \\
\hline Dalea candida & $\begin{array}{l}\text { Petalostemon } \\
\text { candida }\end{array}$ & White prairie clover & Fabaceae - Pea Family & $\mathrm{N}$ & NA & PF \\
\hline Dalea purpurea & $\begin{array}{l}\text { Petalostemon } \\
\text { purpurea }\end{array}$ & Purple prairie clover & Fabaceae - Pea Family & $\mathrm{N}$ & NA & PF \\
\hline $\begin{array}{l}\text { Delphinium carolinianum } \\
\text { subsp. virescens }\end{array}$ & $\begin{array}{l}\text { Delphinium } \\
\text { virescens }\end{array}$ & Prairie larkspur & Helleboraceae - Hellebore Family & $\mathrm{N}$ & NA & PF \\
\hline Dyssodia papposa & & Fetid marigold & Asteraceae - Sunflower Family & $\mathrm{N}$ & NA & PF \\
\hline
\end{tabular}


Table 1. Grassland Plant Community

\begin{tabular}{|c|c|c|c|c|c|c|}
\hline \multicolumn{7}{|c|}{$\begin{array}{c}\text { Plant Species List } \\
\text { NREL South Table Mountain Site }\end{array}$} \\
\hline Scientific Binomial & Synonomy & Common Name & Family & Origin & Season & $\begin{array}{l}\text { Life } \\
\text { Form }\end{array}$ \\
\hline Echinocereus viridiflorus & & Hen-and-chicks & Cactaceae - Cactus Family & $\mathrm{N}$ & NA & SU \\
\hline Echinocerus triglochidialis & & Claret cup & Cactaceae - Cactus Family & $\mathrm{N}$ & NA & SU \\
\hline Elaeagnus angustifolia & & Russian-olive & Elaeagnaceae - Oleaster Family & I & NA & $\mathrm{T}$ \\
\hline $\begin{array}{l}\text { Eleocharis elliptica var. } \\
\text { compressa }\end{array}$ & & Spikerush & Cyperaceae - Sedge Family & $\mathrm{N}$ & NA & $P G$ \\
\hline $\begin{array}{l}\text { Elymus trachycaulus } \\
\text { subsp. trachycaulus }\end{array}$ & $\begin{array}{l}\text { Agropyron caninum } \\
\text { subsp. majus }\end{array}$ & Slender wheatgrass & Poaceae - Grass Family & $\mathrm{N}$ & C & PG \\
\hline Erigeron colo-mexicanus & & Fleabane & Asteraceae - Sunflower Family & $\mathrm{N}$ & NA & PF \\
\hline Erigeron flagellaris & & Daisy fleabane & Asteraceae - Sunflower Family & $\mathrm{N}$ & NA & $\mathrm{BF}$ \\
\hline Eriogonum annuum & & Annual eriogonun & Polygonaceae - Buckwheat Family & $\mathrm{N}$ & NA & AF \\
\hline Eriogonum effusum & & Spreading wild buckwheat & Polygonaceae - Buckwheat Family & $\mathrm{N}$ & NA & PF \\
\hline Eriogonum flavum & & Yellow wild buckwheat & Polygonaceae - Buckwheat Family & $\mathrm{N}$ & NA & PF \\
\hline Eriogonum umbellatum & & Wild buckwheat & Polygonaceae - Buckwheat Family & $\mathrm{N}$ & NA & PF \\
\hline Erodium cicutarium & & Filaree & Geraniaceae - Geranium Family & I & NA & AF \\
\hline Erysimum capitatum & & Western wallflower & Brassicaceae - Mustard Family & $\mathrm{N}$ & NA & $\mathrm{BF}$ \\
\hline $\begin{array}{l}\text { Fraxinus pensylvanica var. } \\
\text { lanceolata }\end{array}$ & & Green ash & Oleaceae - Olive Family & 1 & NA & $\mathrm{T}$ \\
\hline Gaillardia aristata & Gaura parviflora & Blanketflower & Asteraceae - Sunflower Family & $\mathrm{N}$ & NA & PF \\
\hline Gaura mollis & & Gaura & Onagraceae - Evening-primrose & $\mathrm{N}$ & NA & PF \\
\hline
\end{tabular}


Table 1. Grassland Plant Community

\begin{tabular}{|c|c|c|c|c|c|c|}
\hline \multicolumn{7}{|c|}{$\begin{array}{c}\text { Plant Species List } \\
\text { NREL South Table Mountain Site }\end{array}$} \\
\hline Scientific Binomial & Synonomy & Common Name & Family & Origin & Season & $\begin{array}{l}\text { Life } \\
\text { Form }\end{array}$ \\
\hline Gaura parviflora & & Smallflower gaura & Onagraceae - Evening-primrose & $\mathrm{N}$ & NA & AF \\
\hline $\begin{array}{l}\text { Geranium caespitosum } \\
\text { subsp. caespitosum }\end{array}$ & & Wild geranium & Geraniaceae - Geranium Family & $\mathrm{N}$ & NA & PF \\
\hline Grindelia inornata & & Rayless gumweed & Asteraceae - Sunflower Family & $\mathrm{N}$ & NA & PF \\
\hline Grindelia squarrosa & & Curlycup gumweed & Asteraceae - Sunflower Family & $\mathrm{N}$ & NA & $\mathrm{BF}$ \\
\hline Helianthus annuus & & Common sunflower & Asteraceae - Sunflower Family & $\mathrm{N}$ & NA & $\mathrm{AF}$ \\
\hline Helianthus pumilus & & Sunflower & Asteraceae - Sunflower Family & $\mathrm{N}$ & NA & PF \\
\hline Hesperostipa comata & Stipa comata & Needle-and-thread & Poaceae - Grass Family & $\mathrm{N}$ & C & $P G$ \\
\hline Heterotheca villosa & Chrysopsis villosa & Hairy golden aster & Asteraceae - Sunflower Family & $\mathrm{N}$ & NA & SS \\
\hline Lactuca serriola & & Prickly lettuce & Asteraceae - Sunflower Family & I & NA & BF \\
\hline Lesquerella ludoviciana & & Bladderpod & Brassicaceae - Mustard Family & $\mathrm{N}$ & NA & PF \\
\hline Liatris punctata & & Dotted gayfeather & Asteraceae - Sunflower Family & $\mathrm{N}$ & NA & PF \\
\hline $\begin{array}{l}\text { Linaria genistifolia subsp. } \\
\text { dalmatica }\end{array}$ & Linaria dalmatica & Dalmatian toadflax & Scrophulariaceae - Figwort Family & 1 & NA & PF \\
\hline Lithospermum incisum & & Narrowleaf gromwell & Boraginaceae - Borage Family & $\mathrm{N}$ & NA & PF \\
\hline Lupinus argenteus & & Silver lupine & Fabaceae - Pea Family & $\mathrm{N}$ & NA & PF \\
\hline Lygodesmia juncea & & Rush skeletonplant & Asteraceae - Sunflower Family & $\mathrm{N}$ & NA & PF \\
\hline Melilotus officinalis & & Yellow sweetclover & Fabaceae - Pea Family & I & NA & $\mathrm{BF}$ \\
\hline Mertensia lanceolata & & Bluebells & Boraginaceae - Borage Family & $\mathrm{N}$ & NA & PF \\
\hline
\end{tabular}


Table 1. Grassland Plant Community

\begin{tabular}{|c|c|c|c|c|c|c|}
\hline \multicolumn{7}{|c|}{$\begin{array}{l}\text { Plant Species List } \\
\text { NREL South Table Mountain Site }\end{array}$} \\
\hline Scientific Binomial & Synonomy & Common Name & Family & Origin & Season & $\begin{array}{l}\text { Life } \\
\text { Form }\end{array}$ \\
\hline Muhlenbergia richardsonis & & Mat muhly & Poaceae - Grass Family & $\mathrm{N}$ & W & PG \\
\hline Nassella viridula & Stipa viridula & Green needlegrass & Poaceae - Grass Family & $\mathrm{N}$ & C & PG \\
\hline Nuttalia nuda & & Blazingstar & Loasaceae - Loasa Family & $\mathrm{N}$ & NA & PF \\
\hline Oligosporus pacificus & Artemisia campestris & Field sagewort & Asteraceae - Sunflower Family & $\mathrm{N}$ & NA & PF \\
\hline Onopordum acanthium & & Scotch thistle & Asteraceae - Sunflower Family & 1 & NA & $\mathrm{BF}$ \\
\hline Opuntia fragilis & & Brittle cactus & Cactaceae - Cactus Family & $\mathrm{N}$ & NA & SU \\
\hline Opuntia macrorhiza & & Plains prickly pear & Cactaceae - Cactus Family & $\mathrm{N}$ & NA & SU \\
\hline Opuntia phaeacantha & & New Mexican prickly pear & Cactaceae - Cactus Family & $\mathrm{N}$ & NA & SU \\
\hline Opuntia polyacantha & & Plains prickly pear & Cactaceae - Cactus Family & $\mathrm{N}$ & NA & SU \\
\hline Oxytropis lambertii & & Lambert locoweed & Fabaceae - Pea Family & $\mathrm{N}$ & NA & PF \\
\hline $\begin{array}{l}\text { Padus virginiana subsp. } \\
\text { melanocarpa }\end{array}$ & Prunus virginiana & Chokecherry & Rosaceae - Rose Family & $\mathrm{N}$ & NA & $S$ \\
\hline Panicum virgatum & & Switchgrass & Poaceae - Grass Family & $\mathrm{N}$ & W & $P G$ \\
\hline Paronychia jamesii & & James' nailwort & Alsinaceae - Chickweed Family & $\mathrm{N}$ & NA & PF \\
\hline Pascopyrum smithii & Agropyron smithii & Western wheatgrass & Poaceae - Grass Family & $\mathrm{N}$ & C & PG \\
\hline Penstemon virgatus & & Penstemon & Scrophulariaceae - Figwort Family & $\mathrm{N}$ & NA & PF \\
\hline Penstmon angustifolius & & Narrow beardtongue & Scrophulariaceae - Figwort Family & $\mathrm{N}$ & NA & PF \\
\hline Phacelia heterophylla & $\begin{array}{l}\text { Phacelia hastata } \\
\text { var. leucophylla }\end{array}$ & Scorpionweed & Hydrophyllaceae - Waterleaf Family & $\mathrm{N}$ & NA & PF \\
\hline
\end{tabular}


Table 1. Grassland Plant Community

\begin{tabular}{|c|c|c|c|c|c|c|}
\hline \multicolumn{7}{|c|}{$\begin{array}{l}\text { Plant Species List } \\
\text { NREL South Table Mountain Site }\end{array}$} \\
\hline Scientific Binomial & Synonomy & Common Name & Family & Origin & Season & $\begin{array}{l}\text { Life } \\
\text { Form }\end{array}$ \\
\hline Physaria vitulifera & & Fiddle bladderpod & Brassicaceae - Mustard Family & $N$ & NA & PF \\
\hline Physaria vitulifera & & Fiddle bladderpod & Brassicaceae - Mustard Family & $\mathrm{N}$ & NA & PF \\
\hline Plantago patagonica & & Woolly plantain & Plantaginaceae - Plantain Family & $\mathrm{N}$ & NA & $\mathrm{AF}$ \\
\hline Poa compressa & & Canada bluegrass & Poaceae - Grass Family & 1 & C & PG \\
\hline Poa pratensis & & Kentucky bluegrass & Poaceae - Grass Family & I & C & PG \\
\hline Poa secunda & Poa canbyi & Canby bluegrass & Poaceae - Grass Family & $\mathrm{N}$ & C & PG \\
\hline Podospermum laciniatum & Scorzonera laciniata & False salsify & Asteraceae - Sunflower Family & I & NA & $\mathrm{AF}$ \\
\hline Populus deltoides & & Plains cottonwood & Salicaceae - Willow Family & $\mathrm{N}$ & NA & $\mathrm{T}$ \\
\hline Psoralidium tenuiflora & & Slimflower scurfpea & Fabaceae - Pea Family & $\mathrm{N}$ & NA & PF \\
\hline Pterogonum alatum & Erigeron alatum & Winged buckwheat & Polygonaceae - Buckwheat Family & $\mathrm{N}$ & NA & PF \\
\hline Ratibida columnifera & & Prairie coneflower & Asteraceae - Sunflower Family & $\mathrm{N}$ & NA & PF \\
\hline Ratibida tagetes & & Coneflower & Asteraceae - Sunflower Family & $\mathrm{N}$ & NA & PF \\
\hline $\begin{array}{l}\text { Rhus aromatica var. } \\
\text { trilobata }\end{array}$ & & Skunkbrush & Anacardiaceae - Sumac Family & $\mathrm{N}$ & NA & S \\
\hline Rosa arkansana & & Prairie rose & Rosaceae - Rose Family & $\mathrm{N}$ & NA & S \\
\hline Rumex crispus & & Curly dock & Polygonaceae - Buckwheat Family & $\mathrm{N}$ & NA & PF \\
\hline Salsola iberica & & Russian-thistle & $\begin{array}{l}\text { Chenopodiaceae - Goosefoot } \\
\text { Family }\end{array}$ & I & NA & $\mathrm{AF}$ \\
\hline $\begin{array}{l}\text { Schedonnardus } \\
\text { paniculatus }\end{array}$ & & Tumblegrass & Poaceae - Grass Family & $\mathrm{N}$ & $\mathrm{C}$ & PG \\
\hline
\end{tabular}


Table 1. Grassland Plant Community

\begin{tabular}{|c|c|c|c|c|c|c|}
\hline \multicolumn{7}{|c|}{$\begin{array}{c}\text { Plant Species List } \\
\text { NREL South Table Mountain Site }\end{array}$} \\
\hline Scientific Binomial & Synonomy & Common Name & Family & Origin & Season & $\begin{array}{l}\text { Life } \\
\text { Form }\end{array}$ \\
\hline Schizachyrium scoparium & $\begin{array}{l}\text { Andropogon } \\
\text { scoparius }\end{array}$ & Little bluestem & Poaceae - Grass Family & $\mathrm{N}$ & W & PG \\
\hline Scrophularia lanceolata & & Figwort & Scrophulariaceae - Figwort Family & $\mathrm{N}$ & NA & PF \\
\hline Scutellaria brittonii & & Britton's skullcap & Lamiaceae - Mint Family & $\mathrm{N}$ & NA & PF \\
\hline Senecio integerrimus & & Grounsel & Asteraceae - Sunflower Family & $\mathrm{N}$ & NA & $\mathrm{BF} / \mathrm{PF}$ \\
\hline Sisymbrium altissimum & & Tumbling mustard & Brassicaceae - Mustard Family & I & NA & $\mathrm{AF}$ \\
\hline Solidago missouriensis & & Prairie goldenrod & Asteraceae - Sunflower Family & $\mathrm{N}$ & NA & PF \\
\hline Solidago mollis & & Soft goldenrod & Asteraceae - Sunflower Family & $\mathrm{N}$ & NA & PF \\
\hline Sorghastrum nutans & & Indian-grass & Poaceae - Grass Family & $\mathrm{N}$ & W & PG \\
\hline Sphaeralcea coccinea & & Scarlet globemallow & Malvaceae - Mallow Family & $\mathrm{N}$ & NA & PF \\
\hline Sporobolus cryptandrus & & Sand dropseed & Poaceae - Grass Family & $\mathrm{N}$ & W & $P G$ \\
\hline Sporobolus sp. & & Dropseed & Poaceae - Grass Family & $\mathrm{N}$ & W & PG \\
\hline $\begin{array}{l}\text { Symphoricarpos } \\
\text { occidentalis }\end{array}$ & & Western snowberry & $\begin{array}{l}\text { Caprifoliaceae - Honeysuckle } \\
\text { Family }\end{array}$ & $\mathrm{N}$ & NA & S \\
\hline Taraxacum officinale & & Common dandelion & Asteraceae - Sunflower Family & I & NA & PF \\
\hline Thermopsis divaricarpa & & Prairie goldenpea & Fabaceae - Pea Family & $\mathrm{N}$ & NA & PF \\
\hline Tithymalus brachyceras & & Spurge & Euphorbiaceae - Spurge Family & $\mathrm{N}$ & NA & PF \\
\hline Tithymalus montanus & & Spurge & Euphorbiaceae - Spurge Family & $\mathrm{N}$ & NA & PF \\
\hline Toxicodendron rydbergii & & Poison ivy & Anacardiaceae - Sumac Family & $\mathrm{N}$ & NA & S \\
\hline
\end{tabular}


Table 1. Grassland Plant Community

\begin{tabular}{|c|c|c|c|c|c|c|}
\hline \multicolumn{7}{|c|}{$\begin{array}{c}\text { Plant Species List } \\
\text { NREL South Table Mountain Site }\end{array}$} \\
\hline Scientific Binomial & Synonomy & Common Name & Family & Origin & Season & $\begin{array}{l}\text { Life } \\
\text { Form }\end{array}$ \\
\hline Tradescantia occidentalis & & Spiderwort & Commelinaceae & $\mathrm{N}$ & NA & PF \\
\hline Tragia ramosa & & Noseburn & Euphorbiaceae - Spurge Family & $\mathrm{N}$ & NA & PF \\
\hline Tragopogon dubius & & Goatsbeard & Asteraceae - Sunflower Family & I & NA & $\mathrm{BF}$ \\
\hline Ulmus pumila & & Chinese elm & Ulmaceae - Elm Family & I & NA & $\mathrm{T}$ \\
\hline Verbascum thapsus & & Common mullein & Scrophulariaceae - Figwort Family & I & NA & $\mathrm{BF}$ \\
\hline Verbena bracteata & & Prostrate verbena & Verbebaceae - Verbena Family & $\mathrm{N}$ & NA & PF \\
\hline Vicia americana & & American vetch & Fabaceae - Pea Family & $\mathrm{N}$ & NA & PF \\
\hline Yucca glauca & & Yucca & Agavaceae - Agave Family & $\mathrm{N}$ & NA & SU \\
\hline Xanthium strumarium & & Cocklebur & Asteraceae - Sunflower Family & I & NA & $\mathrm{AF}$ \\
\hline
\end{tabular}


Table 2. Upland Shrubland Community

\begin{tabular}{|c|c|c|c|c|c|c|}
\hline \multicolumn{7}{|c|}{$\begin{array}{c}\text { Plant Species List } \\
\text { NREL South Table Mountain Site }\end{array}$} \\
\hline Scientific Binomial & Synonomy & Common Name & Family & Origin & Season & $\begin{array}{l}\text { Life } \\
\text { Form }\end{array}$ \\
\hline Adenolinum lewisii & Linum lewisii & Wild flax & Linaceae - Flax Family & $\mathrm{N}$ & NA & $\mathrm{PF}$ \\
\hline Allium textile & & Wild onion & Alliaceae - Onion Family & $\mathrm{N}$ & NA & PF \\
\hline Alyssum alyssoides & & Pale alyssum & Brassicaceae - Mustard Family & 1 & NA & $\mathrm{AF}$ \\
\hline Alyssum parviflorum & Alyssum minus & Alyssum & Brassicaceae - Mustard Family & I & NA & $\mathrm{AF}$ \\
\hline Amerosedum lanceolatum & & Yellow stonecrop & Crassulaceae - Stonecrop Family & $\mathrm{N}$ & NA & PF \\
\hline Anisantha tectorum & Bromus tectorum & Cheatgrass & Poaceae - Grass Family & I & C & AG \\
\hline Apocynum cannabinum & & Indian hemp & Apocynaceae - Dogbane Family & $\mathrm{N}$ & NA & PF \\
\hline Aristida purpurea & & Three-awn & Poaceae - Grass Family & $\mathrm{N}$ & W & PG \\
\hline Artemisia frigida & & Fringed sagebrush & Asteraceae - Sunflower Family & $\mathrm{N}$ & NA & SS \\
\hline Artemisia ludoviciana & & Prairie sagewort & Asteraceae - Sunflower Family & $\mathrm{N}$ & NA & PF \\
\hline Artemisia tridentata & & Big sagebrush & Asteraceae - Sunflower Family & $\mathrm{N}$ & NA & $\mathrm{S}$ \\
\hline Asclepias viridiflora & & Green flowered milkweed & Asclepiadaceae - Milkweed Family & $\mathrm{N}$ & NA & PF \\
\hline Astragalus drummondii & & Drummonds milk vetch & Fabaceae - Pea Family & $\mathrm{N}$ & NA & PF \\
\hline Atriplex canescens & & Fourwing saltbush & $\begin{array}{l}\text { Chenopodiaceae - Goosefoot } \\
\text { Family }\end{array}$ & $\mathrm{N}$ & NA & $S$ \\
\hline Buchloë dactyloides & & Buffalograss & Poaceae - Grass Family & $\mathrm{N}$ & W & PG \\
\hline Campanula rotundifolia & & Common harebell & Campanulaceae - Bellflower Family & $\mathrm{N}$ & NA & $\mathrm{PF}$ \\
\hline Carduus nutans & & Musk thistle & Asteraceae - Sunflower Family & I & NA & $\mathrm{BF}$ \\
\hline Ceanothus fendleri & & Buckbrush & Rhamnaceae - Buckthorn Family & $\mathrm{N}$ & NA & $S$ \\
\hline Celtis reticulata & & Netleaf hackberry & Ulmaceae - Elm Family & $\mathrm{N}$ & NA & $\mathrm{T}$ \\
\hline Cerastrium strictum & & Mouse-ear & Alsinaceae - Chickweed Family & $\mathrm{N}$ & NA & PF \\
\hline
\end{tabular}


Table 2. Upland Shrubland Community

\begin{tabular}{|c|c|c|c|c|c|c|}
\hline \multicolumn{7}{|c|}{$\begin{array}{c}\text { Plant Species List } \\
\text { NREL South Table Mountain Site }\end{array}$} \\
\hline Scientific Binomial & Synonomy & Common Name & Family & Origin & Season & $\begin{array}{l}\text { Life } \\
\text { Form }\end{array}$ \\
\hline Cercocarpus montanus & & Mountain-mahogany & Rosaceae - Rose Family & $\mathrm{N}$ & NA & S \\
\hline Chondrosum gracile & Bouteloua gracilis & Blue grama & Poaceae - Grass Family & $\mathrm{N}$ & W & PG \\
\hline $\begin{array}{l}\text { Chrysothamnus nauseosus } \\
\text { subsp. graveolens }\end{array}$ & & Rubber rabbitbrush & Asteraceae - Sunflower Family & $\mathrm{N}$ & NA & S \\
\hline Commandra umbellata & & Bastard-toadflax & Santalaceae - Sandelwood Family & $\mathrm{N}$ & NA & PF \\
\hline Critesion jubatum & Hordeum jubatum & Foxtail barley & Poaceae - Grass Family & $\mathrm{N}$ & $\mathrm{C}$ & PG \\
\hline Drymocallis fissa & Potentilla fissa & Cinquefoil & Rosaceae - Rose Family & $\mathrm{N}$ & NA & PF \\
\hline Erigeron colo-mexicanus & & Fleabane & Asteraceae - Sunflower Family & $\mathrm{N}$ & NA & PF \\
\hline Eriogonum effusum & & Spreading wild buckwheat & Polygonaceae - Buckwheat Family & $\mathrm{N}$ & NA & PF \\
\hline Eriogonum umbellatum & & Wild buckwheat & Polygonaceae - Buckwheat Family & $\mathrm{N}$ & NA & PF \\
\hline Erodium cicutarium & & Filaree & Geraniaceae - Geranium Family & I & NA & $\mathrm{AF}$ \\
\hline Gutierrezia sarothrae & & Broom snakeweed & Asteraceae - Sunflower Family & $\mathrm{N}$ & NA & SS \\
\hline Helianthus pumilus & & Sunflower & Asteraceae - Sunflower Family & $\mathrm{N}$ & NA & PF \\
\hline Hesperostipa comata & Stipa comata & Needle-and-thread & Poaceae - Grass Family & $\mathrm{N}$ & C & PG \\
\hline Heterotheca villosa & Chrysopsis villosa & Hairy golden aster & Asteraceae - Sunflower Family & $\mathrm{N}$ & NA & SS \\
\hline $\begin{array}{l}\text { Ipomopsis aggregata subsp. } \\
\text { candida }\end{array}$ & & Gilia & Polemoniaceae - Phlox Family & $\mathrm{N}$ & NA & PF \\
\hline Lathyrus eucosmus & & Elegant peavine & Fabaceae - Pea Family & $\mathrm{N}$ & NA & PF \\
\hline Liatris punctata & & Dotted gayfeather & Asteraceae - Sunflower Family & $\mathrm{N}$ & NA & PF \\
\hline $\begin{array}{l}\text { Linaria genistifolia subsp. } \\
\text { dalmatica }\end{array}$ & Linaria dalmatica & Dalmatian toadflax & Scrophulariaceae - Figwort Family & I & NA & PF \\
\hline
\end{tabular}


Table 2. Upland Shrubland Community

\begin{tabular}{|c|c|c|c|c|c|c|}
\hline \multicolumn{7}{|c|}{$\begin{array}{c}\text { Plant Species List } \\
\text { NREL South Table Mountain Site }\end{array}$} \\
\hline Scientific Binomial & Synonomy & Common Name & Family & Origin & Season & $\begin{array}{l}\text { Life } \\
\text { Form }\end{array}$ \\
\hline Muhlenbergia richardsonis & & Mat muhly & Poaceae - Grass Family & $\mathrm{N}$ & W & PG \\
\hline $\begin{array}{l}\text { Onosmodium molle subsp. } \\
\text { occidentale }\end{array}$ & & Marbleseed & Boraginaceae - Borage Family & $\mathrm{N}$ & NA & PF \\
\hline Opuntia macrorhiza & & Plains prickly pear & Cactaceae - Cactus Family & $\mathrm{N}$ & NA & SU \\
\hline Opuntia polyacantha & & Plains prickly pear & Cactaceae - Cactus Family & $\mathrm{N}$ & NA & SU \\
\hline Oreobatus deliciosus & Rubus deliciousus & Boulder raspberry & Rosaceae - Rose Family & $\mathrm{N}$ & NA & S \\
\hline Oxytropis lambertii & & Lambert locoweed & Fabaceae - Pea Family & $\mathrm{N}$ & NA & PF \\
\hline $\begin{array}{l}\text { Padus virginiana subsp. } \\
\text { melanocarpa }\end{array}$ & Prunus virginiana & Chokecherry & Rosaceae - Rose Family & $\mathrm{N}$ & NA & S \\
\hline Pascopyrum smithii & Agropyron smithii & Western wheatgrass & Poaceae - Grass Family & $\mathrm{N}$ & C & PG \\
\hline Penstemon virgatus & & Penstemon & Scrophulariaceae - Figwort Family & $\mathrm{N}$ & NA & PF \\
\hline Phacelia hastata & & Whiteleaf phacelia & $\begin{array}{l}\text { Hydrophyllaceae } \quad \text { - Water-leaf } \\
\text { Family }\end{array}$ & $\mathrm{N}$ & NA & PF \\
\hline Physaria vitulifera & & Fiddle bladderpod & Brassicaceae - Mustard Family & $\mathrm{N}$ & NA & PF \\
\hline Psoralidium tenuiflora & & Slimflower scurfpea & Fabaceae - Pea Family & $\mathrm{N}$ & NA & PF \\
\hline Rhus aromatica var. trilobata & & Skunkbrush & Anacardiaceae - Sumac Family & $\mathrm{N}$ & NA & $S$ \\
\hline Ribes cereum & & Wax current & Grossulariaceae - Current Family & $\mathrm{N}$ & NA & $S$ \\
\hline Rosa woodsii & & Woods rose & Rosaceae - Rose Family & $\mathrm{N}$ & NA & S \\
\hline Scutellaria brittonii & & Britton's skullcap & Lamiaceae - Mint Family & $\mathrm{N}$ & NA & PF \\
\hline Selaginella densa & & Little club moss & Selaginellaceae - Little Club-moss & $\mathrm{N}$ & NA & PF \\
\hline Sisymbrium altissimum & & Tumbling mustard & Brassicaceae - Mustard Family & I & NA & $\mathrm{AF}$ \\
\hline
\end{tabular}


Table 2. Upland Shrubland Community

\begin{tabular}{|c|c|c|c|c|c|c|}
\hline \multicolumn{7}{|c|}{$\begin{array}{c}\text { Plant Species List } \\
\text { NREL South Table Mountain Site }\end{array}$} \\
\hline Scientific Binomial & Synonomy & Common Name & Family & Origin & Season & $\begin{array}{l}\text { Life } \\
\text { Form }\end{array}$ \\
\hline Sphaeralcea coccinea & & Scarlet globemallow & Malvaceae - Mallow Family & $\mathrm{N}$ & NA & $\mathrm{PF}$ \\
\hline Symphoricarpos occidentalis & & Western snowberry & $\begin{array}{l}\text { Caprifoliaceae - Honeysuckle } \\
\text { Family }\end{array}$ & $\mathrm{N}$ & NA & $S$ \\
\hline Taraxacum officinale & & Common dandelion & Asteraceae - Sunflower Family & I & NA & PF \\
\hline Thlaspi arvense & & Fanweed & Brassicaceae - Mustard Family & I & NA & $\mathrm{AF}$ \\
\hline Toxicodendron rydbergii & & Poison ivy & Anacardiaceae - Sumac Family & N & NA & $S$ \\
\hline Tragopogon dubius & & Goatsbeard & Asteraceae - Sunflower Family & I & NA & $\mathrm{BF}$ \\
\hline Vulpia octoflora & & Six-weeks fescue & Poaceae - Grass Family & $\mathrm{N}$ & $\mathrm{C}$ & $A G$ \\
\hline Yucca glauca & & Yucca & Agavaceae - Agave Family & $\mathrm{N}$ & NA & SU \\
\hline
\end{tabular}


Table 3. Ravine Shrubland Community

Plant Species List

\begin{tabular}{|c|c|c|c|c|c|c|}
\hline \multicolumn{7}{|c|}{$\begin{array}{c}\text { Plant Species List } \\
\text { NREL South Table Mountain Site }\end{array}$} \\
\hline Scientific Binomial & Synonomy & Common Name & Family & Origin & Season & $\begin{array}{l}\text { Life } \\
\text { Form }\end{array}$ \\
\hline Adenolinum lewisii & Linum lewisii & Wild flax & Linaceae - Flax Family & $\mathrm{N}$ & NA & PF \\
\hline Aegilops cylindrica & & Jointed goatgrass & Poaceae - Grass Family & I & C & $A G$ \\
\hline Agropyron cristatum & & Crested wheatgrass & Poaceae - Grass Family & I & C & PG \\
\hline Agrostis stolonifera & & Redtop & Poaceae - Grass Family & 1 & C & PG \\
\hline Alyssum parviflorum & Alyssum minus & Alyssum & Brassicaceae - Mustard Family & 1 & NA & $\mathrm{AF}$ \\
\hline Ambrosia psilostachya & & Western ragweed & Asteraceae - Sunflower Family & $\mathrm{N}$ & NA & PF \\
\hline Anisantha tectorum & Bromus tectorum & Cheatgrass & Poaceae - Grass Family & I & $\mathrm{C}$ & AG \\
\hline Apocynum cannabinum & & Indian hemp & Apocynaceae - Dogbane Family & $\mathrm{N}$ & NA & PF \\
\hline Arctium minus & & Common burdock & Asteraceae - Sunflower Family & 1 & NA & $\mathrm{BF}$ \\
\hline Aristida purpurea & & Three-awn & Poaceae - Grass Family & $\mathrm{N}$ & W & PG \\
\hline Artemisia dracunculus & & Dragon sagewort & Asteraceae - Sunflower Family & $\mathrm{N}$ & NA & PF \\
\hline Artemisia frigida & & Fringed sagebrush & Asteraceae - Sunflower Family & $\mathrm{N}$ & NA & SS \\
\hline Artemisia ludoviciana & & Prairie sagewort & Asteraceae - Sunflower Family & $\mathrm{N}$ & NA & PF \\
\hline Asclepias incarnata & & Swamp milkweed & Asclepiadaceae - Milkweed Family & $\mathrm{N}$ & NA & PF \\
\hline Asclepias pumila & & Plains milkweed & Aclepiadaceae - Milkweed Family & $\mathrm{N}$ & NA & PF \\
\hline Asclepias speciosa & & Showy milkweed & Asclepiadaceae - Milkweed Family & $\mathrm{N}$ & NA & PF \\
\hline Asparagus officinalis & & Asparagus & Asparagaceae - Asparagus Family & $\mathrm{N}$ & NA & PF \\
\hline Aster porteri & & White aster & Asteraceae - Sunflower Family & $\mathrm{N}$ & NA & $\mathrm{PF}$ \\
\hline Aster sp. & & Aster & Asteraceae - Sunflower Family & $\mathrm{N}$ & NA & PF \\
\hline Astragalus drummondii & & Drummonds milk vetch & Fabaceae - Pea Family & $\mathrm{N}$ & NA & PF \\
\hline Bassia sieversiana & Kochia scoparia & Kochia & $\begin{array}{l}\text { Chenopodiaceae - Goosefoot } \\
\text { Family }\end{array}$ & 1 & NA & $\mathrm{AF}$ \\
\hline Bromopis inermis & Bromus inermis & Smooth bromegrass & Poaceae - Grass Family & I & $\mathrm{C}$ & PG \\
\hline
\end{tabular}


Table 3. Ravine Shrubland Community

Plant Species List

NREL South Table Mountain Site

\begin{tabular}{|c|c|c|c|c|c|c|}
\hline Scientific Binomial & Synonomy & Common Name & Family & Origin & Season & $\begin{array}{l}\text { Life } \\
\text { Form }\end{array}$ \\
\hline Calochortus nuttallii & & Sego lily & Liliaceae - Lily Family & $\mathrm{N}$ & NA & PF \\
\hline Camelina microcarpa & & Small-seeded false flax & Brassicaceae - Mustard Family & I & NA & $\mathrm{AF}$ \\
\hline Carduus nutans & & Musk thistle & Asteraceae - Sunflower Family & I & NA & $\mathrm{BF}$ \\
\hline Carex praegracilis & & Slender sedge & Cyperaceae - Sedge Family & $\mathrm{N}$ & NA & $P G$ \\
\hline Carex sp. & & Sedge & Cyperaceae - Sedge Family & $\mathrm{N}$ & NA & PG \\
\hline Celtis reticulata & & Netleaf hackberry & Ulmaceae - Elm Family & $\mathrm{N}$ & NA & $\mathrm{T}$ \\
\hline Centaurea diffusa & Euphorbia & Diffuse knapweed & Asteraceae - Sunflower Family & I & NA & $\mathrm{BF} / \mathrm{PF}$ \\
\hline Chamaesyce glyptopsperma & glyptosperma & Ridgeseed spurge & Euphorbiaceae - Spurge Family & $\mathrm{N}$ & NA & $\mathrm{AF}$ \\
\hline $\begin{array}{l}\text { Chrysothamnus nauseosus } \\
\text { subsp. graveolens }\end{array}$ & & Rubber rabbitbrush & Asteraceae - Sunflower Family & $\mathrm{N}$ & NA & S \\
\hline Cirsium arvense & & Canada thistle & Asteraceae - Sunflower Family & I & NA & PF \\
\hline Clematis ligusticifolia & & Virgin's bower & Ranunculaceae - Buttercup Family & $\mathrm{N}$ & NA & V \\
\hline Conium maculatum & & Poison hemlock & Apiaceae - Parsley Family & I & NA & BF \\
\hline Convolvulus arvensis & & Field bindweed & $\begin{array}{l}\text { Convolvulaceae - Morning Glory } \\
\text { Family }\end{array}$ & I & NA & PF \\
\hline $\begin{array}{l}\text { Crataegus macracantha var. } \\
\text { occidentalis }\end{array}$ & $\begin{array}{l}\text { Crataegus } \\
\text { succulenta }\end{array}$ & Western hawthorn & Rosaceae - Rose Family & $\mathrm{N}$ & NA & $S$ \\
\hline Cynoglossum officinale & & Houndstongue & Boraginaceae - Borage Family & I & NA & $\mathrm{BF}$ \\
\hline Dactylis glomerata & & Orchard grass & Poaceae - Grass Family & 1 & C & PG \\
\hline Dalea candida & $\begin{array}{l}\text { Petalostemon } \\
\text { candida }\end{array}$ & White prairie clover & Fabaceae - Pea Family & $\mathrm{N}$ & NA & PF \\
\hline Descurainia pinnata & & Tansy mustard & Brassicaceae - Mustard Family & $\mathrm{N}$ & NA & AF \\
\hline Descurainia sophia & & Tansy mustard & Brassicaceae - Mustard Family & I & NA & $\mathrm{AF} / \mathrm{BF}$ \\
\hline Dipsacus sylvestris & & Teasel & Dipsacaceae - Teasel Family & I & NA & $\mathrm{BF}$ \\
\hline Echinochloa crusgalli & & Barnyard grass & Poaceae - Grass Family & I & W & AG \\
\hline Elaeagnus angustifolia & & Russian-olive & Elaeagnaceae - Oleaster Family & 1 & NA & $\mathrm{T}$ \\
\hline
\end{tabular}


Table 3. Ravine Shrubland Community

Plant Species List

NREL South Table Mountain Site

\begin{tabular}{|c|c|c|c|c|c|c|}
\hline Scientific Binomial & Synonomy & Common Name & Family & Origin & Season & $\begin{array}{l}\text { Life } \\
\text { Form }\end{array}$ \\
\hline Eleocharis palustris & & Spike-rush & Cyperaceae - Sedge Family & $\mathrm{N}$ & NA & PG \\
\hline Elymus canadensis & & Canada wild rye & Poaceae - Grass Family & $\mathrm{N}$ & C & PG \\
\hline $\begin{array}{l}\text { Elymus lanceolatus subsp. } \\
\text { psammophilus }\end{array}$ & Agropyron riparium & Streambank wheatgrass & Poaceae - Grass Family & $\mathrm{N}$ & $\mathrm{C}$ & PG \\
\hline $\begin{array}{l}\text { Fraxinus pensylvanica var. } \\
\text { lanceolata }\end{array}$ & & Green ash & Oleaceae - Olive Family & I & NA & $\mathrm{T}$ \\
\hline Galium spurium & & Cleavers & Rubiaceae - Madder Family & I & NA & PF \\
\hline Gaura mollis & Gaura parviflora & Gaura & $\begin{array}{l}\text { Onagraceae - Evening-primrose } \\
\text { Family }\end{array}$ & $\mathrm{N}$ & NA & PF \\
\hline Gaura parviflora & & Smallflower gaura & $\begin{array}{l}\text { Onagraceae - Evening-primrose } \\
\text { Family }\end{array}$ & $\mathrm{N}$ & NA & AF \\
\hline Glycyrrhiza lepidota & & American licorice & Fabaceae - Pea Family & $\mathrm{N}$ & NA & $\mathrm{PF}$ \\
\hline Grindelia squarrosa & & Curlycup gumweed & Asteraceae - Sunflower Family & $\mathrm{N}$ & NA & $\mathrm{BF}$ \\
\hline Helianthus pumilus & & Sunflower & Asteraceae - Sunflower Family & $\mathrm{N}$ & NA & $\mathrm{PF}$ \\
\hline Heterotheca villosa & Chrysopsis villosa & Hairy golden aster & Asteraceae - Sunflower Family & $\mathrm{N}$ & NA & SS \\
\hline Juncus arcticus & & Rush & Juncaceae - Rush Family & $\mathrm{N}$ & NA & PG \\
\hline Juncus interior & & Rush & Juncaceae - Rush Family & $\mathrm{N}$ & NA & PG \\
\hline Juncus sp. & & Rush & Juncaceae - Rush Family & $\mathrm{N}$ & NA & PG \\
\hline Lactuca serriola & & Prickly lettuce & Asteraceae - Sunflower Family & I & NA & $\mathrm{BF}$ \\
\hline $\begin{array}{l}\text { Lactuca tatarica subsp. } \\
\text { pulchella }\end{array}$ & & Siberian lettuce & Asteraceae - Sunflower Family & $\mathrm{N}$ & NA & PF \\
\hline Lathyrus eucosmus & & Elegant peavine & Fabaceae - Pea Family & $\mathrm{N}$ & NA & PF \\
\hline Liatris punctata & & Dotted gayfeather & Asteraceae - Sunflower Family & $\mathrm{N}$ & NA & PF \\
\hline $\begin{array}{l}\text { Linaria genistifolia subsp. } \\
\text { dalmatica }\end{array}$ & Linaria dalmatica & Dalmatian toadflax & Scrophulariaceae - Figwort Family & I & NA & PF \\
\hline Mertensia sp. & & Bluebells & Boraginaceae - Borage Family & $\mathrm{N}$ & NA & $\mathrm{PF}$ \\
\hline Medicago sativa & & Alfalfa & Fabaceae - Pea Family & I & NA & $\mathrm{PF}$ \\
\hline Melilotus officinalis & & Yellow sweetclover & Fabaceae - Pea Family & I & NA & $\mathrm{BF}$ \\
\hline
\end{tabular}


Table 3. Ravine Shrubland Community

Plant Species List

NREL South Table Mountain Site

\begin{tabular}{|c|c|c|c|c|c|c|}
\hline Scientific Binomial & Synonomy & Common Name & Family & Origin & Season & $\begin{array}{l}\text { Life } \\
\text { Form }\end{array}$ \\
\hline Melilotus sp. & & Sweetclover & Fabaceae - Pea Family & I & NA & $\mathrm{BF}$ \\
\hline Monarda fistulosa & & Bee balm & Lamiaceae - Mint Family & $\mathrm{N}$ & NA & PF \\
\hline Muhlenbergia richardsonis & & Mat muhly & Poaceae - Grass Family & $\mathrm{N}$ & W & PG \\
\hline Negundo aceroides & Acer negundo & Box elder & Aceraceae - Maple Family & $\mathrm{N}$ & NA & $\mathrm{T}$ \\
\hline Nepeta cataria & & Catnip & Lamiaceae - Mint Family & I & NA & PF \\
\hline Opuntia polyacantha & & Plains prickly pear & Cactaceae - Cactus Family & $\mathrm{N}$ & NA & SU \\
\hline Oxybaphus nyctagineus & Mirabilis nyctaginea & Wild four-o'clocks & $\begin{array}{l}\text { Nyctaginaceae - Four-o'clock } \\
\text { Family }\end{array}$ & $\mathrm{N}$ & NA & PF \\
\hline $\begin{array}{l}\text { Padus virginiana subsp. } \\
\text { melanocarpa }\end{array}$ & Prunus virginiana & Chokecherry & Rosaceae - Rose Family & $\mathrm{N}$ & NA & S \\
\hline Panicum capillare & & Witchgrass & Poaceae - Grass Family & $\mathrm{N}$ & W & $A G$ \\
\hline Panicum virgatum & & Switchgrass & Poaceae - Grass Family & $\mathrm{N}$ & W & PG \\
\hline Pascopyrum smithii & Agropyron smithii & Western wheatgrass & Poaceae - Grass Family & $\mathrm{N}$ & C & PG \\
\hline Persicaria maculata & $\begin{array}{l}\text { Polygonum } \\
\text { persicaria }\end{array}$ & Lady's thumb & Polygonaceae - Buckwheat Family & 1 & NA & AF \\
\hline Phacelia heterophylla & $\begin{array}{l}\text { Phacelia hastata } \\
\text { var. } \\
\text { leucophylla }\end{array}$ & Scorpionweed & Hydrophyllaceae - Waterleaf Family & $\mathrm{N}$ & NA & PF \\
\hline Phleum pratense & & Common Timothy & Poaceae - Grass Family & 1 & C & PG \\
\hline Physalis virginiana & & Virginia ground-cherry & Solanaceae - Nightshade Family & I & NA & PF \\
\hline Plantago major & & Common plantain & Plantaginaceae - Plantain Family & I & NA & PF \\
\hline Poa compressa & & Canada bluegrass & Poaceae - Grass Family & I & C & PG \\
\hline Poa pratensis & & Kentucky bluegrass & Poaceae - Grass Family & I & $\mathrm{C}$ & PG \\
\hline Populus deltoides & & Plains cottonwood & Salicaceae - Willow Family & $\mathrm{N}$ & NA & $\mathrm{T}$ \\
\hline Populus $x$ acuminata & & & Salicaceae - Willow Family & $\mathrm{N}$ & NA & $\mathrm{T}$ \\
\hline Prunus americana & & Wild plum & Rosaceae - Rose Family & $\mathrm{N}$ & NA & $S$ \\
\hline Psoralidium tenuiflora & & Slimflower scurfpea & Fabaceae - Pea Family & $\mathrm{N}$ & NA & PF \\
\hline
\end{tabular}


Table 3. Ravine Shrubland Community

Plant Species List

\begin{tabular}{|c|c|c|c|c|c|c|}
\hline \multicolumn{7}{|c|}{$\begin{array}{c}\text { Plant Species List } \\
\text { NREL South Table Mountain Site }\end{array}$} \\
\hline Scientific Binomial & Synonomy & Common Name & Family & Origin & Season & $\begin{array}{c}\text { Life } \\
\text { Form }\end{array}$ \\
\hline Pyrus malus & Malus pumila & Apple & Rosaceae - Rose Family & I & NA & $\mathrm{T}$ \\
\hline $\begin{array}{l}\text { Ranculus abortivus subsp. } \\
\text { acrolasius }\end{array}$ & & Buttercup & Ranunculaceae - Buttercup Family & $\mathrm{N}$ & NA & PF \\
\hline Ratibida columnifera & & Prairie coneflower & Asteraceae - Sunflower Family & $\mathrm{N}$ & NA & PF \\
\hline Rhus aromatica var. trilobata & & Skunkbrush & Anacardiaceae - Sumac Family & $\mathrm{N}$ & NA & S \\
\hline Ribes aureum & & Golden current & Grossulariaceae - Current Family & $\mathrm{N}$ & NA & S \\
\hline Ribes cereum & & Wax current & Grossulariaceae - Current Family & $\mathrm{N}$ & NA & S \\
\hline Rosa arkansana & & Prairie rose & Rosaceae - Rose Family & $\mathrm{N}$ & NA & S \\
\hline Rosa woodsii & & Woods rose & Rosaceae - Rose Family & $\mathrm{N}$ & NA & S \\
\hline Rumex crispus & & Curly dock & Polygonaceae - Buckwheat Family & $\mathrm{N}$ & NA & PF \\
\hline Salix amygdaloides & & Peach-leaf willow & Salicaceae - Willow Family & $\mathrm{N}$ & NA & $\mathrm{T}$ \\
\hline Salix exigua & & Sandbar willow & Salicaceae - Willow Family & $\mathrm{N}$ & NA & S \\
\hline Salix fragilis & & Crack willow & Salicaceae - Willow Family & I & NA & $\mathrm{T}$ \\
\hline Salix lutea & & Yellow willow & Salicaceae - Willow Family & $\mathrm{N}$ & NA & S \\
\hline Shepherdia argentea & & Silverberry & Elaeagnaceae - Oleaster Family & $\mathrm{N}$ & NA & S \\
\hline Sisymbrium altissimum & & Tumbling mustard & Brassicaceae - Mustard Family & I & NA & $\mathrm{AF}$ \\
\hline Solidago mollis & & Soft goldenrod & Asteraceae - Sunflower Family & $\mathrm{N}$ & NA & $\mathrm{PF}$ \\
\hline Sphaeralcea coccinea & & Scarlet globemallow & Malvaceae - Mallow Family & $\mathrm{N}$ & NA & PF \\
\hline Stipa viridula & & Green needlegrass & Poaceae - Grass Family & $\mathrm{N}$ & $\mathrm{C}$ & PG \\
\hline Symphoricarpos occidentalis & & Western snowberry & $\begin{array}{l}\text { Caprifoliaceae - Honeysuckle } \\
\text { Family }\end{array}$ & $\mathrm{N}$ & NA & $S$ \\
\hline Taraxacum officinale & & Common dandelion & Asteraceae - Sunflower Family & I & NA & $\mathrm{PF}$ \\
\hline Thlaspi arvense & & Fanweed & Brassicaceae - Mustard Family & 1 & NA & $\mathrm{AF}$ \\
\hline Toxicodendron rydbergii & & Poison ivy & Anacardiaceae - Sumac Family & $\mathrm{N}$ & NA & S \\
\hline
\end{tabular}


Table 3. Ravine Shrubland Community

\begin{tabular}{|c|c|c|c|c|c|c|}
\hline \multicolumn{7}{|c|}{$\begin{array}{c}\text { Plant Species List } \\
\text { NREL South Table Mountain Site }\end{array}$} \\
\hline Scientific Binomial & Synonomy & Common Name & Family & Origin & Season & $\begin{array}{l}\text { Life } \\
\text { Form }\end{array}$ \\
\hline Tragia ramosa & & Noseburn & Euphorbiaceae - Spurge Family & $\mathrm{N}$ & NA & PF \\
\hline Tragopogon dubius & & Goatsbeard & Asteraceae - Sunflower Family & I & NA & $\mathrm{BF}$ \\
\hline Typha angustifolia & & Narrow-leaved cattail & Typhaceae - Cattail Family & $\mathrm{N}$ & NA & PF \\
\hline Typha latifolia & & Common cattail & Typhaceae - Cattail Family & $\mathrm{N}$ & NA & PF \\
\hline Ulmus pumila & & Chinese elm & Ulmaceae - Elm Family & I & NA & $\mathrm{T}$ \\
\hline Verbascum thapsus & & Common mullein & Scrophulariaceae - Figwort Family & 1 & NA & $\mathrm{BF}$ \\
\hline Veronica catenata & & Speedwell & Scrophulariaceae - Figwort Family & $\mathrm{N}$ & NA & PF \\
\hline Vicia americana & & American vetch & Fabaceae - Pea Family & $\mathrm{N}$ & NA & PF \\
\hline Yucca glauca & & Yucca & Agavaceae - Agave Family & $\mathrm{N}$ & NA & SU \\
\hline
\end{tabular}


Table 4. Wetland Community

Plant Species List

NREL South Table Mountain Site

\begin{tabular}{|c|c|c|c|c|c|c|}
\hline Scientific Binomial & Synonomy & Common Name & Family & Origin & Season & $\begin{array}{l}\text { Life } \\
\text { Form }\end{array}$ \\
\hline Asclepias incarnata & & Swamp milkweed & Asclepiadaceae - Milkweed Family & $\mathrm{N}$ & NA & PF \\
\hline Asclepias speciosa & & Showy milkweed & Asclepiadaceae - Milkweed Family & $\mathrm{N}$ & NA & PF \\
\hline Carex praegracilis & & Slender sedge & Cyperaceae - Sedge Family & $\mathrm{N}$ & NA & PG \\
\hline Carex sp. & & Sedge & Cyperaceae - Sedge Family & $\mathrm{N}$ & NA & PG \\
\hline Carex utriculata & Carex rostrata & Sedge & Cyperaceae - Sedge Family & $\mathrm{N}$ & NA & PG \\
\hline Celtis reticulata & & Netleaf hackberry & Ulmaceae - Elm Family & $\mathrm{N}$ & NA & $\mathrm{T}$ \\
\hline Clematis ligusticifolia & & Virgin's bower & Ranunculaceae - Buttercup Family & $\mathrm{N}$ & NA & V \\
\hline Comiun maculatum & & Poison hemlock & Apiaceae - Parsley Family & I & NA & $\mathrm{BF}$ \\
\hline Convolvulus arvensis & & Field bindweed & $\begin{array}{l}\text { Convolvulaceae - Morning glory } \\
\text { Family }\end{array}$ & 1 & NA & PF \\
\hline Critesion jubatum & Hordeum jubatum & Foxtail barley & Poaceae - Grass Family & $\mathrm{N}$ & C & PG \\
\hline Cynoglossum officinale & & Houndstongue & Boraginaceae - Borage Family & I & NA & $\mathrm{BF}$ \\
\hline $\begin{array}{l}\text { Eleocharis elliptica var. } \\
\text { compressa }\end{array}$ & & Spikerush & Cyperaceae - Sedge Family & $\mathrm{N}$ & NA & PG \\
\hline Dipsacus sylvestris & & Teasel & Dipsacaceae - Teasel Family & 1 & NA & $\mathrm{BF}$ \\
\hline Eleocharis palustris & & Spike-rush & Cyperaceae - Sedge Family & $\mathrm{N}$ & NA & PG \\
\hline $\begin{array}{l}\text { Elymus lanceolatus subsp. } \\
\text { psammophilus }\end{array}$ & $\begin{array}{l}\text { Agropyron } \\
\text { riparium }\end{array}$ & Streambank wheatgrass & Poaceae - Grass Family & $\mathrm{N}$ & C & $P G$ \\
\hline $\begin{array}{l}\text { Fraxinus pensylvanica var. } \\
\text { lanceolata }\end{array}$ & & Green ash & Oleaceae - Olive Family & I & NA & $\mathrm{T}$ \\
\hline Glycyrrhiza lepidota & & American licorice & Fabaceae - Pea Family & $\mathrm{N}$ & NA & PF \\
\hline Juncus arcticus & & Rush & Juncaceae - Rush Family & $\mathrm{N}$ & NA & $P G$ \\
\hline
\end{tabular}


Table 4. Wetland Community

Plant Species List

NREL South Table Mountain Site

\begin{tabular}{|c|c|c|c|c|c|c|}
\hline Scientific Binomial & Synonomy & Common Name & Family & Origin & Season & $\begin{array}{l}\text { Life } \\
\text { Form }\end{array}$ \\
\hline Juncus balticus & & Baltic rush & Juncaceae - Rush Family & $\mathrm{N}$ & NA & $P G$ \\
\hline Juncus interior & & Rush & Juncaceae - Rush Family & $\mathrm{N}$ & NA & PG \\
\hline Juncus sp. & & Rush & Juncaceae - Rush Family & $\mathrm{N}$ & NA & PG \\
\hline Lithospermum incisum & & Narrowleaf gromwell & Boraginaceae - Borage Family & $\mathrm{N}$ & NA & PF \\
\hline Melilotus sp. & & Sweetclover & Fabaceae - Pea Family & I & NA & $\mathrm{BF}$ \\
\hline Mentha arvensis & & Fieldmint & Lamiaceae - Mint Family & $\mathrm{N}$ & NA & PF \\
\hline Mertensia lanceolata & & Bluebells & Boraginaceae - Borage Family & $\mathrm{N}$ & NA & PF \\
\hline Monarda fistulosa & & Bee balm & Lamiaceae - Mint Family & $\mathrm{N}$ & NA & PF \\
\hline Muhlenbergia richardsonis & & Mat muhly & Poaceae - Grass Family & $\mathrm{N}$ & W & PG \\
\hline Nepeta cataria & & Catnip & Lamiaceae - Mint Family & I & NA & PF \\
\hline $\begin{array}{l}\text { Padus virginiana subsp. } \\
\text { melanocarpa }\end{array}$ & Prunus virginiana & Chokecherry & Rosaceae - Rose Family & $\mathrm{N}$ & NA & $S$ \\
\hline Panicum virgatum & & Switchgrass & Poaceae - Grass Family & $\mathrm{N}$ & W & PG \\
\hline Pascopyrum smithii & Agropyron smithii & Western wheatgrass & Poaceae - Grass Family & $\mathrm{N}$ & $\mathrm{C}$ & PG \\
\hline Poa compressa & & Canada bluegrass & Poaceae - Grass Family & I & $\mathrm{C}$ & PG \\
\hline Poa pratensis & & Kentucky bluegrass & Poaceae - Grass Family & 1 & C & PG \\
\hline Populus deltoides & & Plains cottonwood & Salicaceae - Willow Family & $\mathrm{N}$ & NA & $\mathrm{T}$ \\
\hline Populus $x$ acuminata & Prunus americana & Wild plum & $\begin{array}{l}\text { Salicaceae - } \quad \text { Willow } \\
\text { Rosaceae - Rose Family }\end{array}$ & N N & NA NA & TS \\
\hline Ribes aureum & & Golden current & Grossulariaceae - Current Family & $\mathrm{N}$ & NA & $S$ \\
\hline
\end{tabular}


Table 4. Wetland Community

Plant Species List

NREL South Table Mountain Site

\begin{tabular}{|c|c|c|c|c|c|c|}
\hline Scientific Binomial & Synonomy & Common Name & Family & Origin & Season & $\begin{array}{l}\text { Life } \\
\text { Form }\end{array}$ \\
\hline Ribes cereum & & Wax current & Grossulariaceae - Current Family & $\mathrm{N}$ & NA & S \\
\hline Rosa woodsii & & Woods rose & Rosaceae - Rose Family & $\mathrm{N}$ & NA & $S$ \\
\hline Rumex sp. & & Dock & Polygonaceae - Buckwheat Family & $\mathrm{N}$ & NA & PF \\
\hline Salix amygdaloides & & Peach-leaf willow & Salicaceae - Willow Family & $\mathrm{N}$ & NA & $\mathrm{T}$ \\
\hline Salix exigua & & Sandbar willow & Salicaceae - Willow Family & $\mathrm{N}$ & NA & S \\
\hline Salix fragilis & & Crack willow & Salicaceae - Willow Family & I & NA & $\mathrm{T}$ \\
\hline Salix lutea & & Yellow willow & Salicaceae - Willow Family & $\mathrm{N}$ & NA & $S$ \\
\hline Scirpus sp. & & Bulrush & Cyperaceae - Sedge Family & $\mathrm{N}$ & NA & PG \\
\hline Shepherdia argentea & & Silverberry & Elaeagnaceae - Oleaster Family & $\mathrm{N}$ & NA & $S$ \\
\hline Symphoricarpos occidentalis & & Western snowberry & $\begin{array}{lll}\text { Caprifoliaceae } \quad- & \text { Honeysuckle } \\
\text { Family } & & \end{array}$ & $\mathrm{N}$ & NA & $S$ \\
\hline Taraxacum officinale & & Common dandelion & Asteraceae - Sunflower Family & I & NA & PF \\
\hline Typha angustifolia & & Narrow-leaved cattail & Typhaceae - Cattail Family & $\mathrm{N}$ & NA & PF \\
\hline Typha latifolia & & Common cattail & Typhaceae - Cattail Family & $\mathrm{N}$ & NA & PF \\
\hline Ulmus pumila & & Chinese elm & Ulmaceae - Elm Family & 1 & NA & $\mathrm{T}$ \\
\hline Veronica Americana & & American brooklime & Scrophulariaceae - Figwort Family & $\mathrm{N}$ & NA & $A F$ \\
\hline Veronica catenata & & Speedwell & Scrophulariaceae - Figwort Family & $\mathrm{N}$ & NA & PF \\
\hline Vibernum lantana & & Wayfaring tree & Caryophyllaceae - Pink Family & I & NA & $S$ \\
\hline
\end{tabular}


Table 5. Disturbed/Reclaimed Plant Community

\begin{tabular}{|c|c|c|c|c|c|c|}
\hline \multicolumn{7}{|c|}{$\begin{array}{c}\text { Plant Species List } \\
\text { NREL South Table Mountain Site }\end{array}$} \\
\hline Scientific Binomial & Synonomy & Common Name & Family & Origin & Season & $\begin{array}{l}\text { Life } \\
\text { Form }\end{array}$ \\
\hline Agropyron cristatum & & Crested wheatgrass & Poaceae - Grass Family & I & C & PG \\
\hline Agropyron intermedium & & Intermediate wheatgrass & Poaceae - Grass Family & 1 & $\mathrm{C}$ & $P G$ \\
\hline Alyssum parviflorum & Alyssum minus & Alyssum & Brassicaceae - Mustard Family & I & NA & $A F$ \\
\hline Ambrosia psilostachya & & Western ragweed & Asteraceae - Sunflower Family & $\mathrm{N}$ & NA & PF \\
\hline Ambrosia trifida & & Giant ragweed & Asteraceae - Sunflower Family & I & NA & $\mathrm{AF}$ \\
\hline Andropogon gerardii & & Big bluestem & Poaceae - Grass Family & $\mathrm{N}$ & W & PG \\
\hline Anisantha tectorum & Bromus tectorum & Cheatgrass & Poaceae - Grass Family & 1 & C & $A G$ \\
\hline Arctium minus & & Common burdock & Asteraceae - Sunflower Family & 1 & NA & BF \\
\hline Aristida purpurea & & Three-awn & Poaceae - Grass Family & $\mathrm{N}$ & W & PG \\
\hline Artemisia frigida & & Fringed sagebrush & Asteraceae - Sunflower Family & $\mathrm{N}$ & NA & SS \\
\hline Artemisia ludoviciana & & Prairie sagewort & Asteraceae - Sunflower Family & $\mathrm{N}$ & NA & PF \\
\hline Aster porteri & & White aster & Asteraceae - Sunflower Family & $\mathrm{N}$ & NA & PF \\
\hline Atriplex canescens & & Fourwing saltbush & $\begin{array}{l}\text { Chenopodiaceae - Goosefoot } \\
\text { Family }\end{array}$ & $\mathrm{N}$ & NA & $S$ \\
\hline Bassia sieversiana & Kochia scoparia & Kochia & $\begin{array}{l}\text { Chenopodiaceae }- \text { Goosefoot } \\
\text { Family }\end{array}$ & 1 & NA & AF \\
\hline Bouteloua curtipendula & & Side-oats grama & Poaceae - Grass Family & $\mathrm{N}$ & W & PG \\
\hline Breea arvensis & Circium arvense & Canada thistle & Asteraceae - Sunflower Family & 1 & NA & PF \\
\hline Brickellia eupatorioides & & Brickellia & Asteraceae - Sunflower Family & $\mathrm{N}$ & NA & PF \\
\hline Bromopis inermis & Bromus inermis & Smooth bromegrass & Poaceae - Grass Family & I & C & PG \\
\hline
\end{tabular}


Table 5. Disturbed/Reclaimed Plant Community

\begin{tabular}{|c|c|c|c|c|c|c|}
\hline \multicolumn{7}{|c|}{$\begin{array}{c}\text { Plant Species List } \\
\text { NREL South Table Mountain Site }\end{array}$} \\
\hline Scientific Binomial & Synonomy & Common Name & Family & Origin & Season & $\begin{array}{l}\text { Life } \\
\text { Form }\end{array}$ \\
\hline Bromus japonicus & & Japanese brome & Poaceae - Grass Family & I & C & $A G$ \\
\hline Buchloë dactyloides & & Buffalograss & Poaceae - Grass Family & $\mathrm{N}$ & W & $P G$ \\
\hline Acosta diffusa & Centaurea diffusa & Diffuse knapweed & Asteraceae - Sunflower Family & 1 & NA & $\mathrm{BF} / \mathrm{PF}$ \\
\hline Cardaria draba & & Whitetop & Brassicaceae - Mustard Family & 1 & NA & PF \\
\hline Chamaesyce glyptopsperma & $\begin{array}{l}\text { Euphorbia } \\
\text { glyptosperma }\end{array}$ & Ridgeseed spurge & Euphorbiaceae - Spurge Family & $\mathrm{N}$ & NA & AF \\
\hline Chenopodium album & & Common lambsquarters & $\begin{array}{l}\text { Chenopodiaceae - Goosefoot } \\
\text { Family }\end{array}$ & I & NA & AF \\
\hline Chondrosum gracile & Bouteloua gracilis & Blue grama & Poaceae - Grass Family & $\mathrm{N}$ & W & $P G$ \\
\hline $\begin{array}{l}\text { Chrysothamnus nauseosus } \\
\text { subsp. graveolens }\end{array}$ & & Rubber rabbitbrush & Asteraceae - Sunflower Family & $\mathrm{N}$ & NA & $S$ \\
\hline Comiun maculatum & & Poison hemlock & Apiaceae - Parsley Family & 1 & NA & BF \\
\hline Convolvulus arvensis & & Field bindweed & $\begin{array}{l}\text { Convolvulaceae - Morning Glory } \\
\text { Family }\end{array}$ & 1 & NA & PF \\
\hline Critesion jubatum & Hordeum jubatum & Foxtail barley & Poaceae - Grass Family & $\mathrm{N}$ & $\mathrm{C}$ & $P G$ \\
\hline Dyssodia papposa & & Fetid marigold & Asteraceae - Sunflower Family & $\mathrm{N}$ & NA & PF \\
\hline Echinochloa crusgalli & & Barnyard grass & Poaceae - Grass Family & I & W & $A G$ \\
\hline Elaeagnus angustifolia & & Russian-olive & Elaeagnaceae - Oleaster Family & 1 & NA & $\mathrm{T}$ \\
\hline Epilobium brachycarpum & $\begin{array}{l}\text { Epilobium } \\
\text { paniculatum }\end{array}$ & Willowherb & $\begin{array}{l}\text { Onagraceae - Evening-primrose } \\
\text { Family }\end{array}$ & $\mathrm{N}$ & NA & AF \\
\hline Erodium cicutarium & & Filaree & Geraniaceae - Geranium Family & I & NA & AF \\
\hline Festuca sp. & & Fescue & Poaceae - Grass Family & $\mathrm{N}$ & C & PG \\
\hline Glycyrrhiza lepidota & & American licorice & Fabaceae - Pea Family & $\mathrm{N}$ & NA & PF \\
\hline
\end{tabular}


Table 5. Disturbed/Reclaimed Plant Community

\begin{tabular}{|c|c|c|c|c|c|c|}
\hline \multicolumn{7}{|c|}{$\begin{array}{c}\text { Plant Species List } \\
\text { NREL South Table Mountain Site }\end{array}$} \\
\hline Scientific Binomial & Synonomy & Common Name & Family & Origin & Season & $\begin{array}{l}\text { Life } \\
\text { Form }\end{array}$ \\
\hline Gutierrezia sarothrae & & Broom snakeweed & Asteraceae - Sunflower Family & $\mathrm{N}$ & NA & SS \\
\hline Helianthus annuus & & Common sunflower & Asteraceae - Sunflower Family & $\mathrm{N}$ & NA & $\mathrm{AF}$ \\
\hline Hesperostipa comata & Stipa comata & Needle-and-thread & Poaceae - Grass Family & $\mathrm{N}$ & C & PG \\
\hline Lactuca serriola & & Prickly lettuce & Asteraceae - Sunflower Family & I & NA & $\mathrm{BF}$ \\
\hline $\begin{array}{l}\text { Linaria genistifolia subsp. } \\
\text { dalmatica }\end{array}$ & Linaria dalmatica & Dalmatian toadflax & Scrophulariaceae - Figwort Family & I & NA & PF \\
\hline Lophopyrum elongatum & $\begin{array}{l}\text { Agropyron } \\
\text { elongatum }\end{array}$ & Tall wheatgrass & Poaceae - Grass Family & I & C & PG \\
\hline Malva neglecta & & Common mallow & Malvaceae - Mallow Family & 1 & NA & PF \\
\hline Melilotus officinalis & & Yellow sweetclover & Fabaceae - Pea Family & 1 & NA & $\mathrm{BF}$ \\
\hline Muhlenbergia richardsonis & & Mat muhly & Poaceae - Grass Family & $\mathrm{N}$ & W & PG \\
\hline Negundo aceroides & Acer negundo & Box elder & Aceraceae - Maple Family & $\mathrm{N}$ & NA & $\mathrm{T}$ \\
\hline Nepeta cataria & & Catnip & Lamiaceae - Mint Family & I & NA & PF \\
\hline Onopordum acanthium & & Scotch thistle & Asteraceae - Sunflower Family & I & NA & $\mathrm{BF}$ \\
\hline Oxybaphus linearis & Mirabilis linearis & Narrowleaf umbrellawort & $\begin{array}{l}\text { Nyctaginaceae } \\
\text { Family }\end{array}$ & $\mathrm{N}$ & NA & PF \\
\hline Panicum capillare & & Witchgrass & Poaceae - Grass Family & $\mathrm{N}$ & W & $A G$ \\
\hline Panicum virgatum & & Switchgrass & Poaceae - Grass Family & $\mathrm{N}$ & W & PG \\
\hline Pascopyrum smithii & Agropyron smithii & Western wheatgrass & Poaceae - Grass Family & $\mathrm{N}$ & C & PG \\
\hline Phleum pratense & & Common Timothy & Poaceae - Grass Family & I & $\mathrm{C}$ & PG \\
\hline Physalis virginiana & & Virginia ground-cherry & Solanaceae - Nightshade Family & I & NA & PF \\
\hline
\end{tabular}


Table 5. Disturbed/Reclaimed Plant Community

\begin{tabular}{|c|c|c|c|c|c|c|}
\hline \multicolumn{7}{|c|}{$\begin{array}{c}\text { Plant Species List } \\
\text { NREL South Table Mountain Site }\end{array}$} \\
\hline Scientific Binomial & Synonomy & Common Name & Family & Origin & Season & $\begin{array}{l}\text { Life } \\
\text { Form }\end{array}$ \\
\hline Pinus edulis & & Pinon pine & Pinaceae - Pine Family & $\mathrm{N}$ & NA & $\mathrm{T}$ \\
\hline Pinus ponderosa & & Ponderosa pine & Pinaceae - Pine Family & $\mathrm{N}$ & NA & $\mathrm{T}$ \\
\hline Poa pratensis & & Kentucky bluegrass & Poaceae - Grass Family & 1 & $\mathrm{C}$ & $P G$ \\
\hline Poinsettia dentata & Euphorbia dentata & Toothed spurge & Euphorbiaceae - Spurge Family & I & NA & $\mathrm{AF}$ \\
\hline Populus deltoides & & Plains cottonwood & Salicaceae - Willow Family & $\mathrm{N}$ & NA & $\mathrm{T}$ \\
\hline Psoralidium tenuiflora & & Slimflower scurfpea & Fabaceae - Pea Family & $\mathrm{N}$ & NA & PF \\
\hline Sabina scopulorum & & Rocky mountain juniper & Cupressaceae - Cypress Family & $\mathrm{N}$ & NA & $\mathrm{T}$ \\
\hline Salix amygdaloides & & Peach-leaf willow & Salicaceae - Willow Family & $\mathrm{N}$ & NA & $\mathrm{T}$ \\
\hline Salsola iberica & & Russian-thistle & $\begin{array}{l}\text { Chenopodiaceae - Goosefoot } \\
\text { Family }\end{array}$ & 1 & NA & AF \\
\hline Schizachyrium scoparium & $\begin{array}{l}\text { Andropogon } \\
\text { scoparius }\end{array}$ & Little bluestem & Poaceae - Grass Family & $\mathrm{N}$ & W & PG \\
\hline Setaria viridis & & Green floxtail & Poaceae - Grass Family & I & W & $A G$ \\
\hline Seteria glauca & & Yellow foxtail & Poaceae - Grass Family & I & NA & $A G$ \\
\hline Solidago missouriensis & & Prairie goldenrod & Asteraceae - Sunflower Family & $\mathrm{N}$ & NA & PF \\
\hline Sorghastrum nutans & & Indian-grass & Poaceae - Grass Family & $\mathrm{N}$ & W & PG \\
\hline Sphaeralcea coccinea & & Scarlet globemallow & Malvaceae - Mallow Family & $\mathrm{N}$ & NA & PF \\
\hline Stipa viridula & & Green needlegrass & Poaceae - Grass Family & $\mathrm{N}$ & C & PG \\
\hline Taraxicum officinale & & Commondandelion & Asteraceae - Sunflower Family & 1 & NA & PF \\
\hline Tragopogon dubius & & Goatsbeard & Asteraceae - Sunflower Family & I & NA & BF \\
\hline
\end{tabular}


Table 5. Disturbed/Reclaimed Plant Community

\begin{tabular}{|c|c|c|c|c|c|c|}
\hline \multicolumn{7}{|c|}{$\begin{array}{c}\text { Plant Species List } \\
\text { NREL South Table Mountain Site }\end{array}$} \\
\hline Scientific Binomial & Synonomy & Common Name & Family & Origin & Season & $\begin{array}{l}\text { Life } \\
\text { Form }\end{array}$ \\
\hline Tribulus terrestris & & Puncturevine & Zygophyllaceae - Caltrop Family & 1 & NA & $A F$ \\
\hline Ulmus pumila & & Chinese elm & Ulmaceae - Elm Family & 1 & NA & $\mathrm{T}$ \\
\hline Verbascum thapsus & & Common mullein & Scrophulariaceae - Figwort Family & I & NA & $\mathrm{BF}$ \\
\hline
\end{tabular}




\section{APPENDIX B}

\section{Photos}




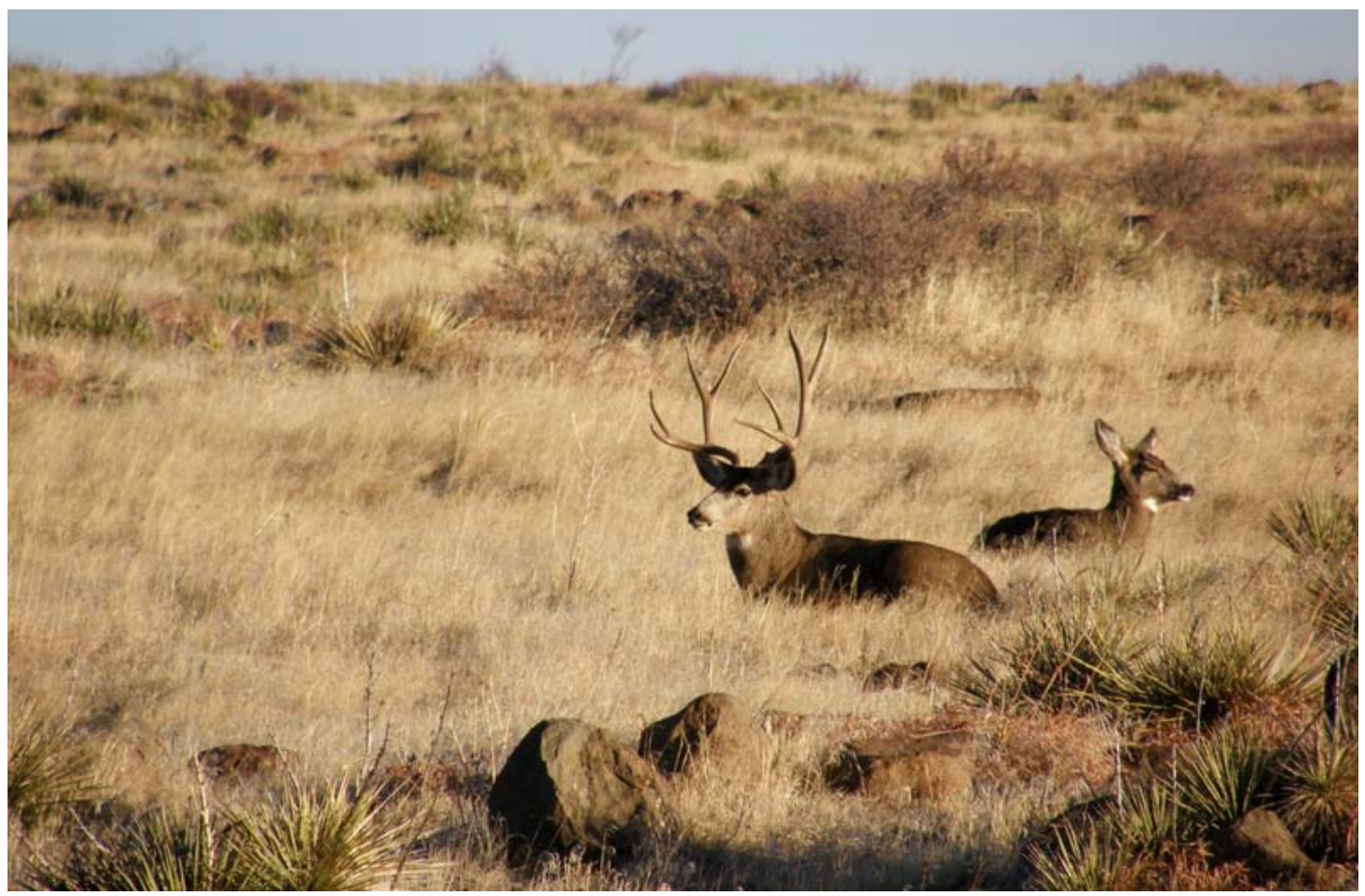

Photo 1. December 6, 2010. Short-grass grassland and tall shrubland (upper right). Two mule deer are resting in the grassland on the South Table mesa top. A pocket of mountain mahagony can be seen beyond the deer.

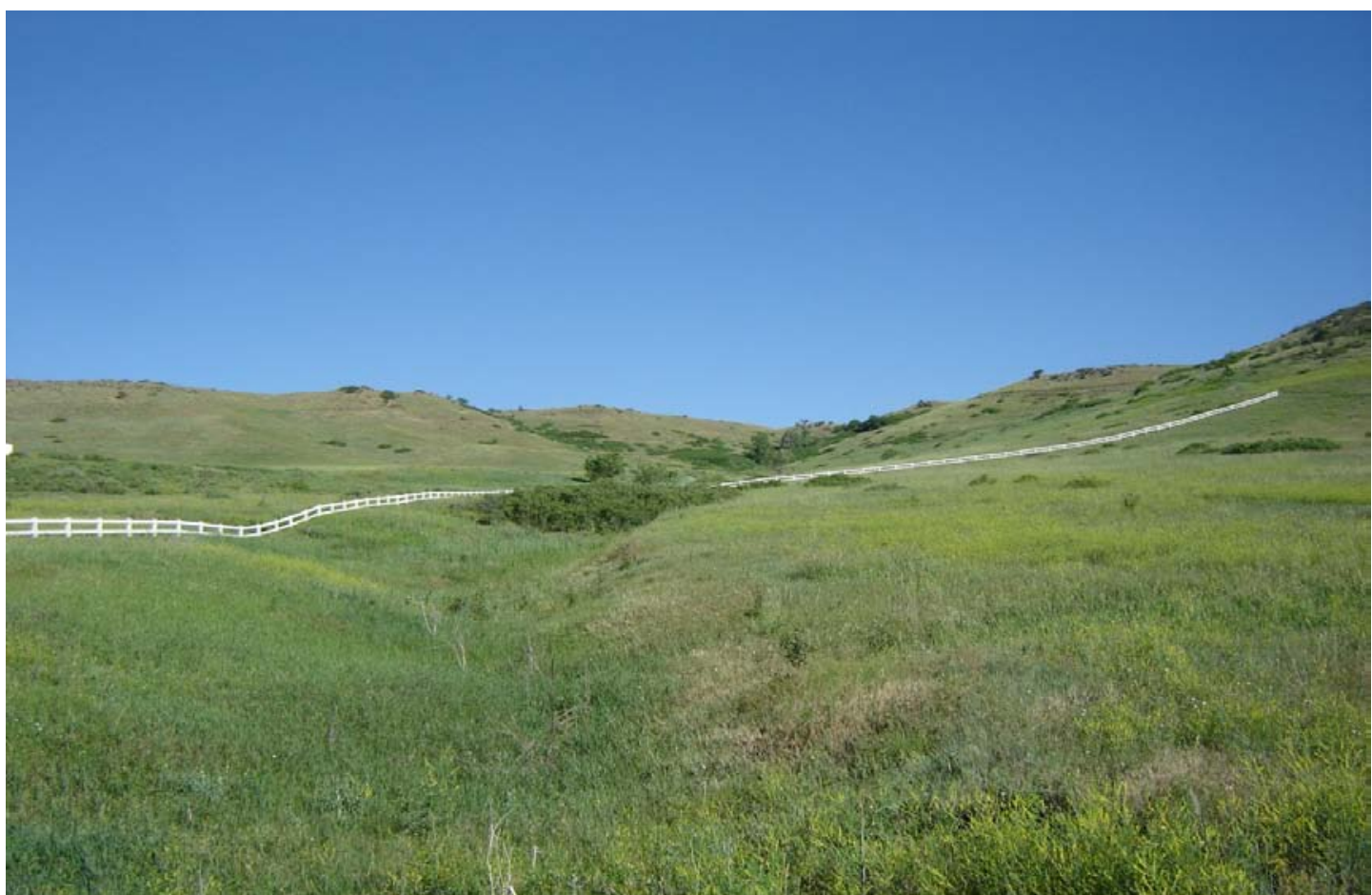

Photo 2. June 23, 2010. Mixed-grass grassland north of the Visitor Center. On the rolling slopes and the flat land on either side of the ephemeral drainage, mixed grass grassland is the dominant plant community. In the upper reaches of the drainage, ravine shrublands are surrounded by grassland and grade into grassland plants toward the lower reaches of the east drainage. 


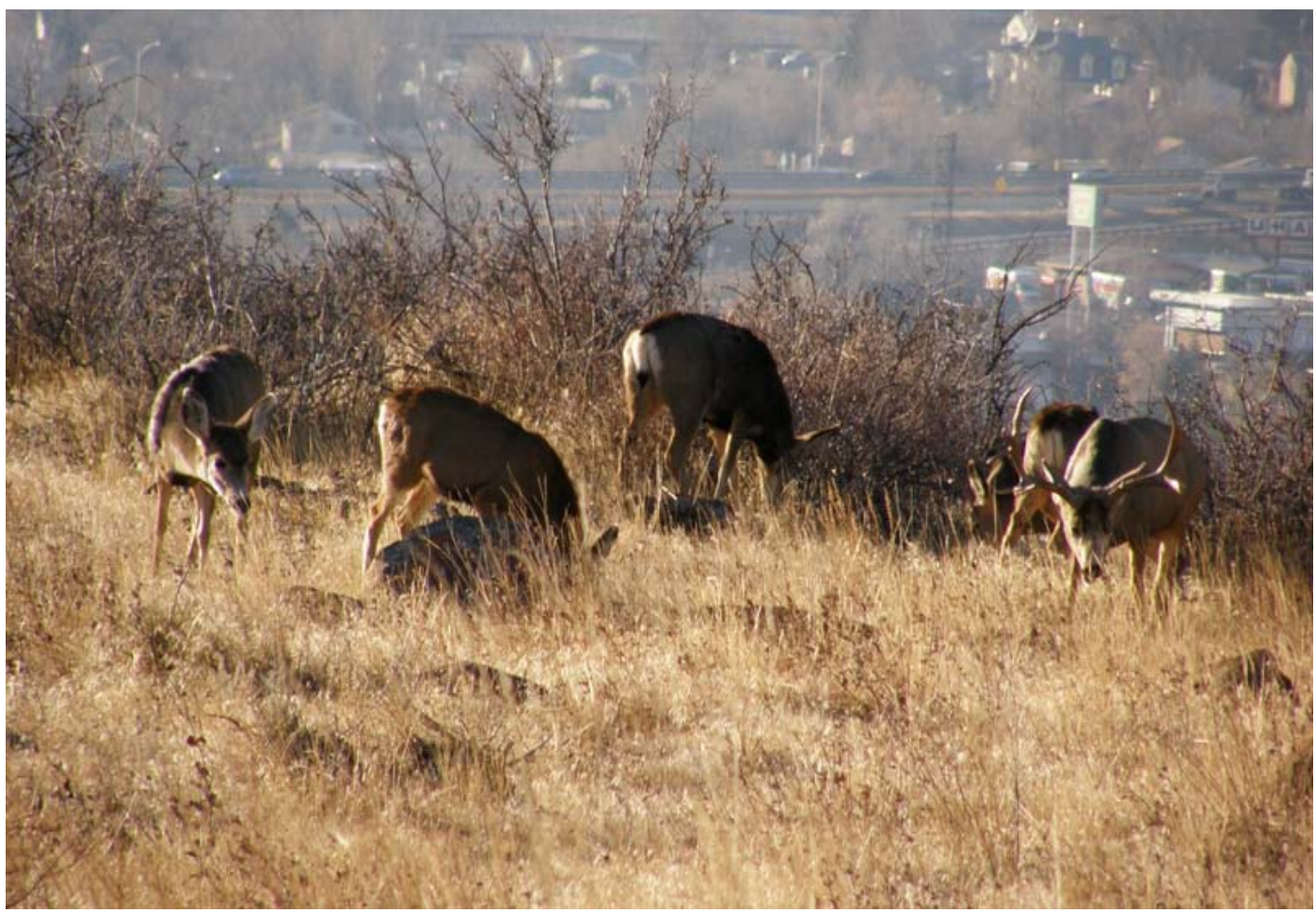

Photo 3. December 6, 2010. Tall shrubland (background) and short-grass grassland (foreground) on the mesa top, upslope and northeast of the amphitheatre drainage. Several species of upland tall shrubs, dominated by mountain mahagony can be seen beyond the grazing mule deer.

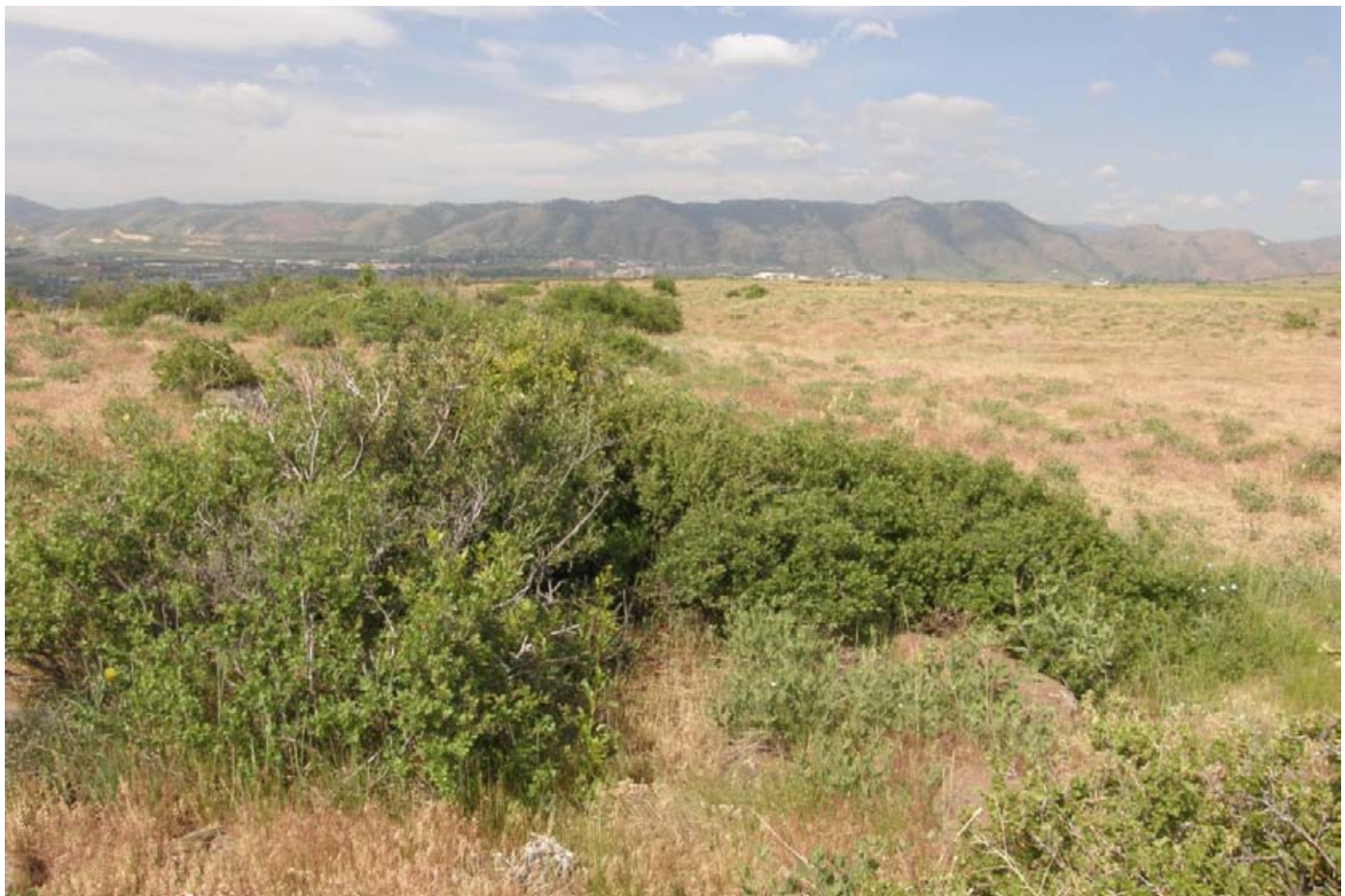

Photo 4. June 9, 2010. Short shrubland along the east project boundary. One tall shrub of mountain mahagony was observed but this community is dominated by skunkbrush in this area. 


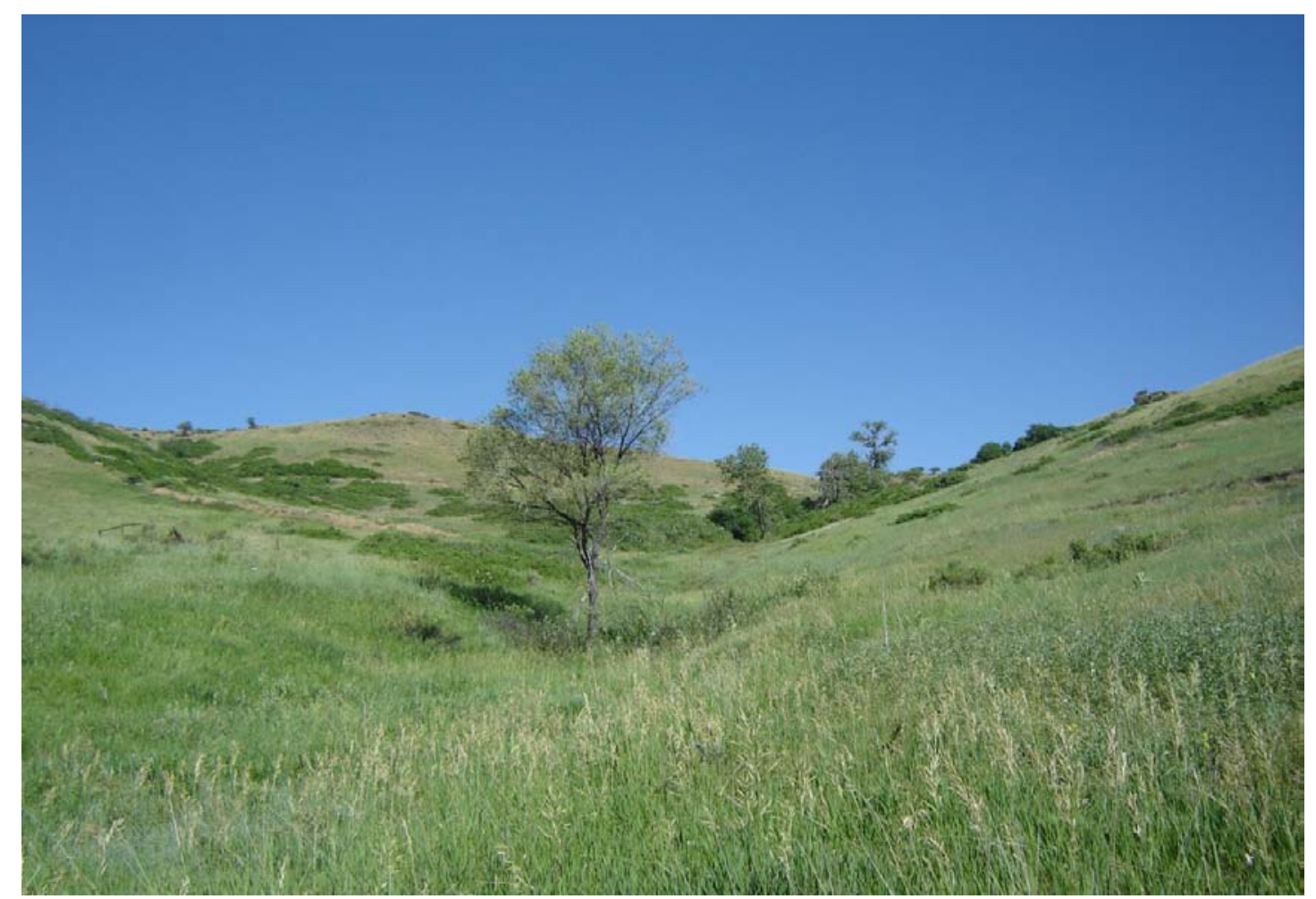

Photo 5. June 23, 2010. Ravine shrubland along the upgradient portions of the east drainage north of the Visitor Center.

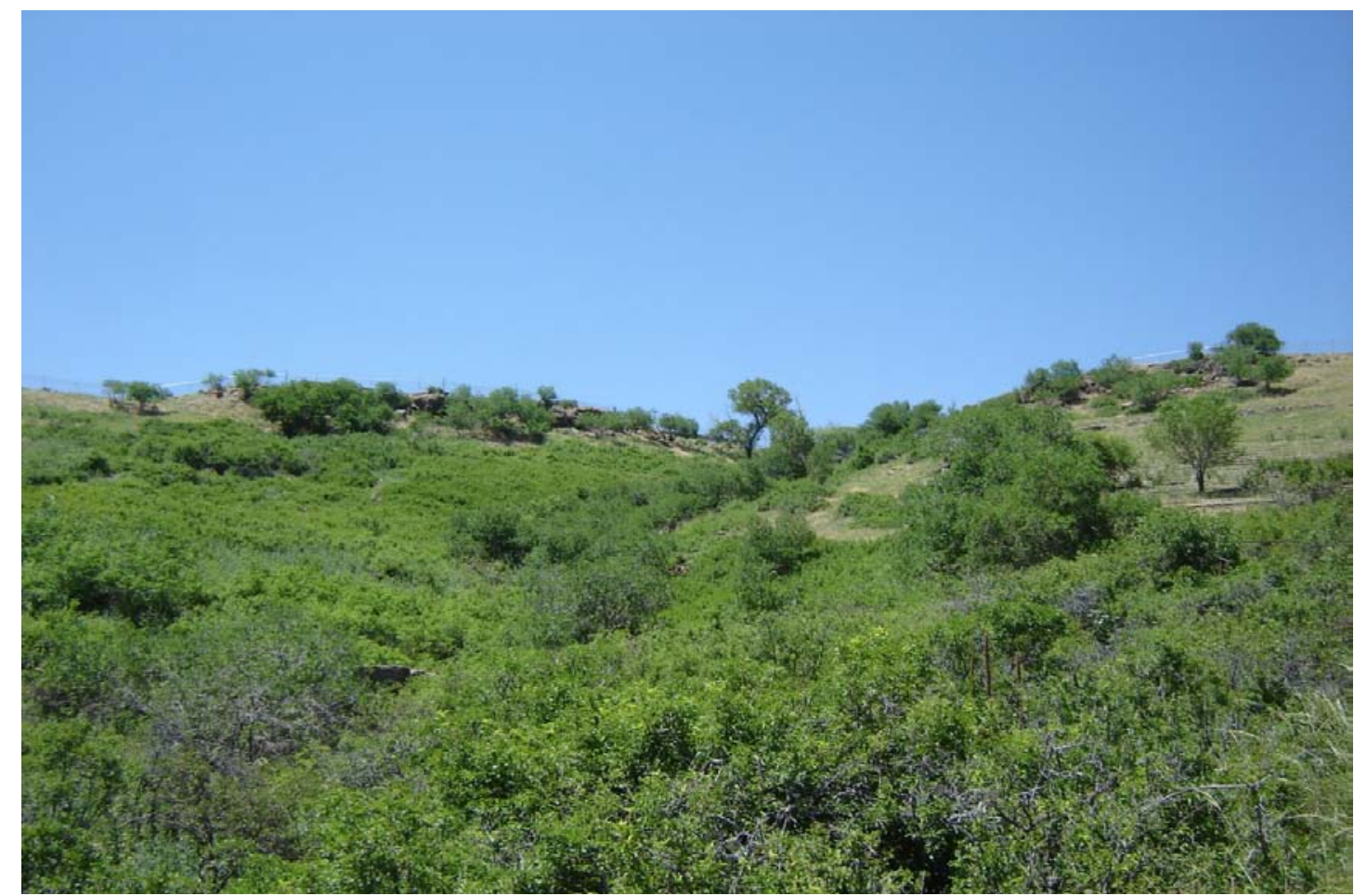

Photo 6. June 23, 2010. Ravine shrubland overstory in the upper reaches of the amphitheatre drainage 


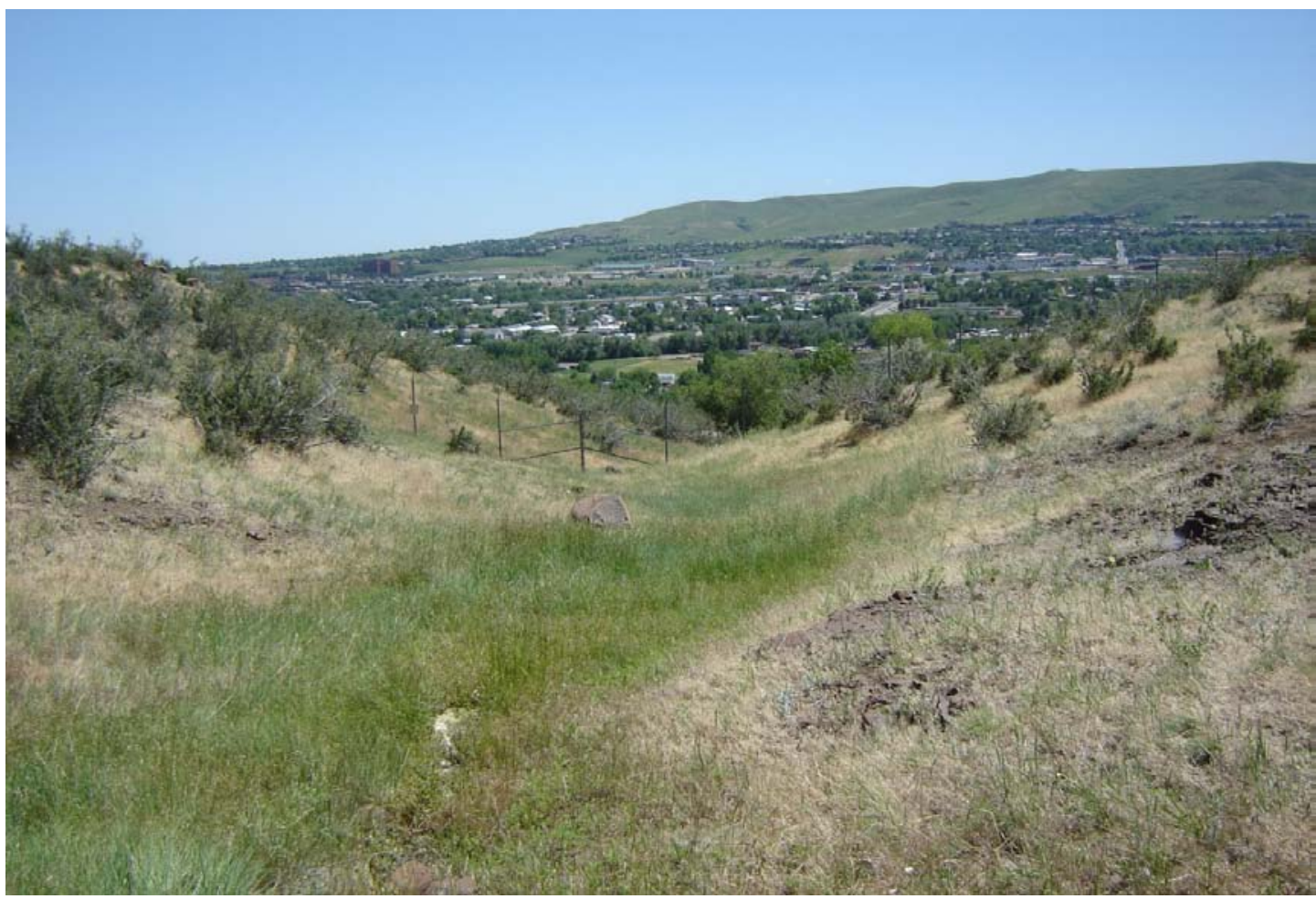

Photo 7. June 23, 2010. Wetland at the top of the drainage in the southwest of the project boundary.

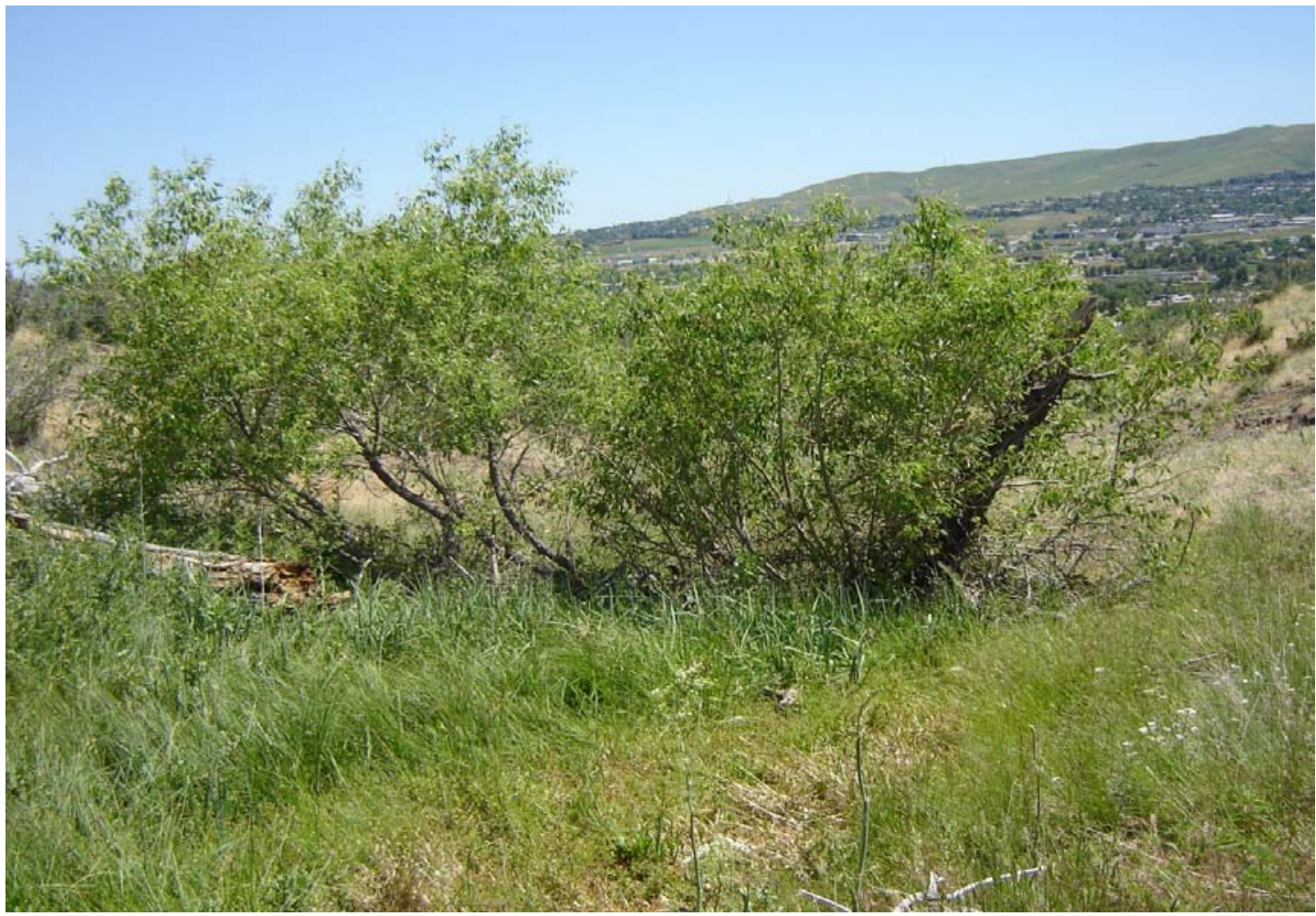

Photo 8. June 23, 2010. Same wetland as in Photo 7 (above) slightly further upgradient with various obligate wetland graminoids in the foreground and peachleaf willows in the background. 


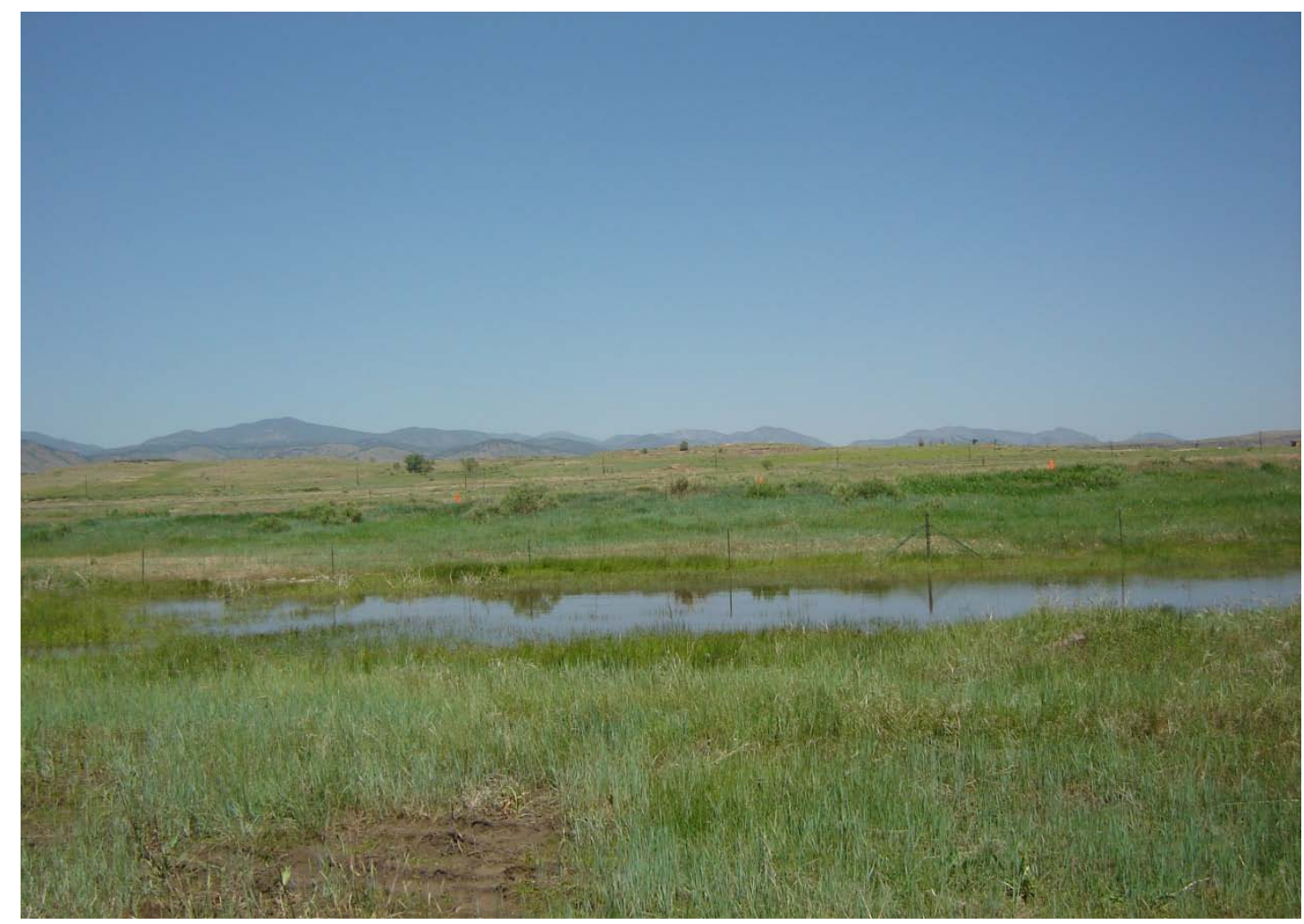

Photo 9. June 23, 2010. Linear depressional wetland northeast of the solar facilty along the northern project boundary.

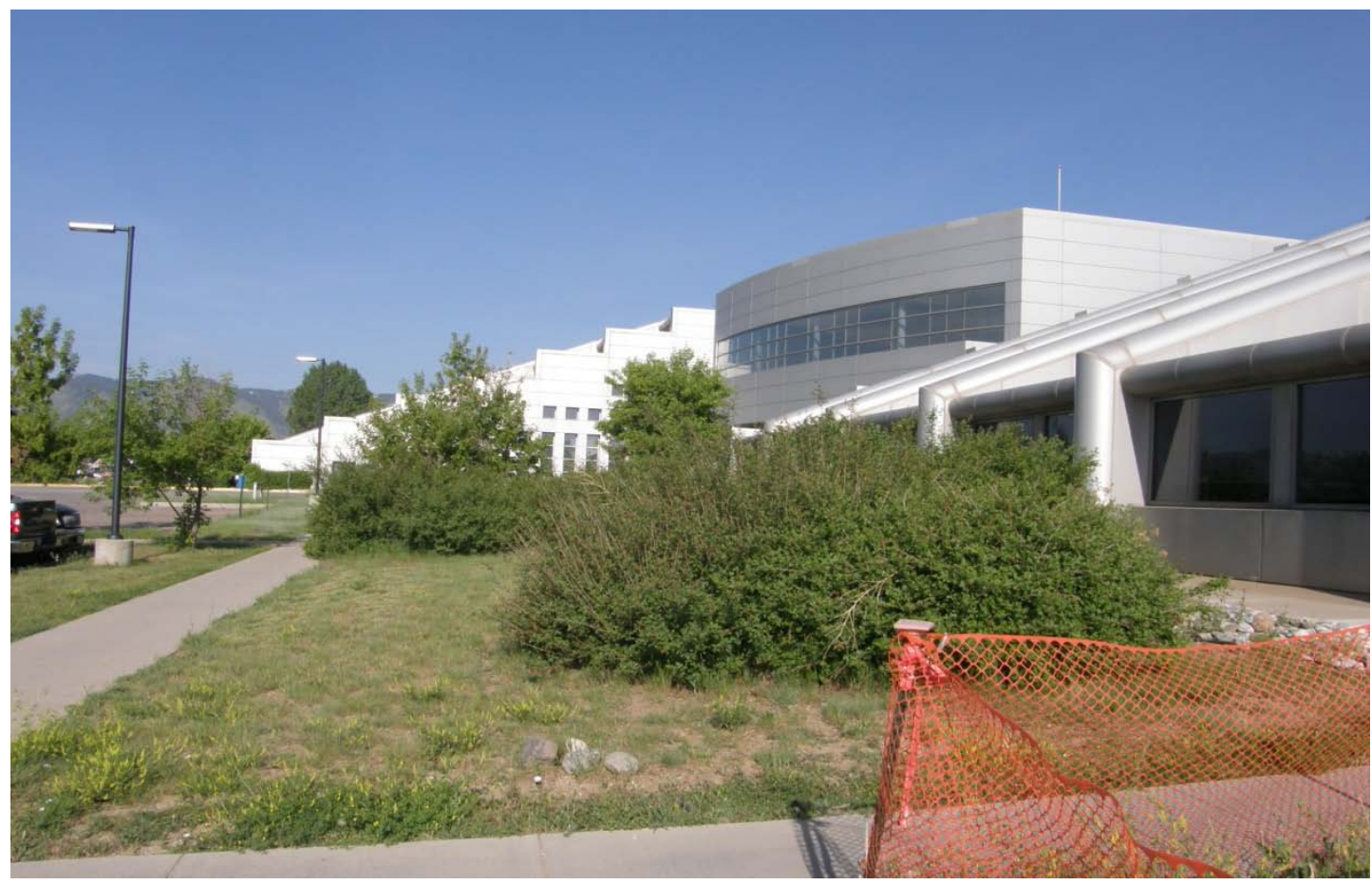

Photo 10. June 9, 2010. A disturbed community on the perimeter of the Solar Energy Research Facility near the central portion of the southern project boundary. 


\section{APPENDIX C}

Colorado Natural Heritage Program Data Query Response 
June 25, 2010

Janetta Shepard, P.W.S.

Restoration Ecologist

Walsh Environmental Scientists \& Engineers, LLC

4888 Pearl East Circle, Suite 108

Boulder, Colorado 80301
Knowledge to Go Places

\section{$\underset{\substack{\text { Ctate } \\ \text { Cniversily }}}{\text { Colorado }}$}

\section{Colorado Natural Heritage Program}

Colorado State University 8002 Campus Delivery

Fort Collins, Colorado 80523-8002

(970) 491-1309

FAX: (970) 491-3349

www.cnhp.colostate.edu

Dear Janetta:

The Colorado Natural Heritage Program (CNHP) is in receipt of your request for information regarding the NREL South Table Mountain site of interest in Jefferson County, Colorado. In response, I have searched our Biodiversity Tracking and Conservation System (BIOTICS) for natural heritage elements (occurrences of significant natural communities and rare, threatened or endangered plants and animals) documented from the vicinity of the area specified in your request, specifically within a two-mile buffered radius of the proposed project area as described by the shapefile provided to CNHP for the purposes of this review.

The enclosed report describes natural heritage resources known from this area and gives location (by Township, Range, and Section), precision information, and the date of last observation of the element at that location. This report includes elements known to occur within the specified project site, as well as elements known from similar landscapes near the site. Please note that "precision" reflects the resolution of original data. For example, an herbarium record from " 4 miles east of Colorado Springs" provides much less spatial information than a topographic map showing the exact location of the occurrence. "Precision" codes of

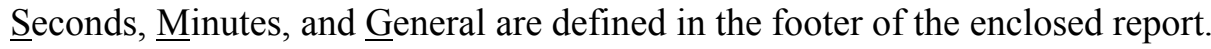

The report also outlines the status of known elements. We have included status according to Natural Heritage Program methodology and legal status under state and federal statutes. Natural Heritage ranks are standardized across the Heritage Program network, and are assigned for global and state levels of rarity. They range from " 1 " for critically imperiled or extremely rare elements, to " 5 " for those that are demonstrably secure.

You may notice that some occurrences do not have sections listed. Those species have been designated as "sensitive" due to their rarity and threats by human activity. Peregrine falcons, for example, are susceptible to human breeders removing falcon eggs from their nests. For these species, CNHP does not normally provide location information beyond township and range. Please contact us should you require more detailed information for sensitive occurrences.

There are no CNHP designated Potential Conservation Area (PCAs) and no Network of Conservation Areas (NCAs) located within the project area, but multiple PCAs are in the general vicinity (see enclosed shapefile and PCA site reports). In order to successfully protect populations or occurrences, it is necessary to delineate conservation areas. These conservation areas focus on capturing the ecological processes that are necessary to support the continued existence of a particular element of natural heritage significance. Conservation areas may include a single occurrence of a rare element or a suite of rare elements or significant features.

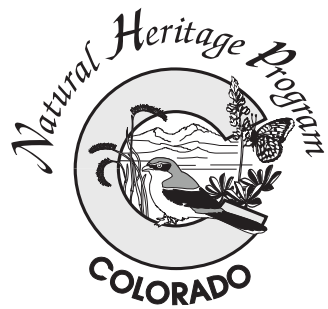


The goal of the process is to identify a land area that can provide the habitat and ecological processes upon which a particular element or suite of elements depends for their continued existence. The best available knowledge of each species' life history is used in conjunction with information about topographic, geomorphic, and hydrologic features, vegetative cover, as well as current and potential land uses. The proposed boundary does not automatically exclude all activity. It is hypothesized that some activities will cause degradation to the element or the process on which they depend, while others will not. Consideration of specific activities or land use changes proposed within or adjacent to the preliminary conservation planning boundary should be carefully considered and evaluated for their consequences to the element on which the conservation unit is based.

The Colorado Division of Wildlife has legal authority over wildlife in the state. CDOW would therefore be responsible for the evaluation of and final decisions regarding any potential effects a proposed project may have on wildlife. If you would like more specific information regarding these or other vertebrate species in the vicinity of the area of interest, please contact the Colorado Division of Wildlife.

The information contained herein represents the results of a search of Colorado Natural Heritage Program's (CNHP) Biodiversity Tracking and Conservation System (BIOTICS), and can be used as notice to anticipate possible impacts or identify areas of interest. Care should be taken in interpreting these data. Sensitive elements are currently known from within the vicinity of the proposed project area (see enclosed Adobe PDF species report). Additionally, we also searched our observations database for species watch-listed but not fully tracked by CNHP and found no observations in the vicinity of the project area. Please note that the absence of data for a particular area, species, or habitat does not necessarily mean that these natural heritage resources do not occur on or adjacent to the project site, rather that our files do not currently contain information to document their presence. CNHP information should not replace field studies necessary for more localized planning efforts, especially if impacts to wildlife habitat are possible.

Although every attempt is made to provide the most current and precise information possible, please be aware that some of our sources provide a higher level of accuracy than others, and some interpretation may be required. CNHP's data system is constantly updated and revised. Please contact CNHP for an update or assistance with interpretation of this natural heritage information.

The data contained in the report is the product and property of the Colorado Natural Heritage Program (CNHP), a sponsored program at Colorado State University (CSU). The data contained herein are provided on an as is, as available basis without warranties of any kind, expressed or implied, including (but not limited to) warranties of merchantability, fitness for a particular purpose, and non-infringement. CNHP, CSU and the state of Colorado further expressly disclaim any warranty that the data are error free or current as of the date supplied.

Sincerely,

Michael Menefee

Environmental Review Coordinator

Enc. 
Locations and Status of Rare and/or Imperiled Species and Natural Communities known from or likely to occur within a two-mile radius of 327 acres at NREL's South Table Mountain facility in Jefferson County, CO

Report generated: 25 June 2010

Copyright $@$ 2010. Colorado State University. Colorado Natural Heritage Program. All Rights Reserved.

\begin{tabular}{|c|c|c|c|c|c|c|c|c|c|c|c|c|c|c|}
\hline EO_ID & major group & scientific name & common name & Prec & last obs & $\begin{array}{l}\text { Town/ } \\
\text { Range }\end{array}$ & Sec & TRS Note & grank & srank & $\begin{array}{l}\text { eo- } \\
\text { rank }\end{array}$ & $E S A$ & fed stat & st stat \\
\hline 8,530 & Birds & Seiurus aurocapillus & Ovenbird & G & 1989-07-04 & 004S070W & 07 & & G5 & $\mathrm{S} 2 \mathrm{~B}$ & $\mathrm{H}$ & - & & \\
\hline 8,251 & Insects & Atrytone arogos & Arogos Skipper & M & 1979-99-99 & 004S070W & 13 & & G3 & $\mathrm{S} 2$ & $\mathrm{H}$ & - & & \\
\hline \multirow[t]{9}{*}{9,371} & Insects & Atrytone arogos & Arogos Skipper & M & $1991-08-10$ & 003S070W & 27 & & G3 & $\mathrm{S} 2$ & E & - & & \\
\hline & & & & & & 003S070W & 28 & & & & & & & \\
\hline & & & & & & 0035070W & 29 & & & & & & & \\
\hline & & & & & & 0035070W & 32 & & & & & & & \\
\hline & & & & & & 003S070W & 33 & & & & & & & \\
\hline & & & & & & 003S070W & 34 & & & & & & & \\
\hline & & & & & & 004S070W & 03 & & & & & & & \\
\hline & & & & & & 004S070W & 04 & & & & & & & \\
\hline & & & & & & 004S070W & 05 & & & & & & & \\
\hline 8,014 & Insects & $\begin{array}{l}\text { Callophrys mossii } \\
\text { schryveri }\end{array}$ & Moss's Elfin & G & $1969-04-20$ & 0035070W & 20 & & G4T3 & $\mathrm{S} 2 \mathrm{~S} 3$ & $\mathrm{H}$ & - & & \\
\hline 5,505 & Insects & $\begin{array}{l}\text { Callophrys mossii } \\
\text { schryveri }\end{array}$ & Moss's Elfin & M & $1982-04-11$ & 0035070W & 33 & & G4T3 & $\mathrm{S} 2 \mathrm{~S} 3$ & $\mathrm{H}$ & - & & \\
\hline 9,842 & Insects & Doa ampla & A Moth & M & 9999-99-99 & 004S070W & 04 & & GNR & S1 & E & - & & \\
\hline 6,874 & Insects & Erynnis martialis & Mottled Dusky Wing & G & $1980-04-27$ & 0035070W & 20 & & G3 & $\mathrm{S} 2 \mathrm{~S} 3$ & $\mathrm{H}$ & - & & \\
\hline 202 & Insects & Grammia sp. 1 & A Tiger Moth & M & 9999-99-99 & 004S070W & 04 & & $\mathrm{G} 2 \mathrm{G} 3$ & SNR & E & - & & \\
\hline 11,317 & Insects & Polites origenes & Cross-line Skipper & G & 1984-07-03 & $0035071 \mathrm{~W}$ & 36 & & G5 & s3 & $\mathrm{H}$ & - & & \\
\hline 9,600 & $\begin{array}{l}\text { Vascular } \\
\text { Plants }\end{array}$ & $\begin{array}{l}\text { Aquilegia } \\
\text { saximontana }\end{array}$ & $\begin{array}{l}\text { Rocky Mountain } \\
\text { columbine }\end{array}$ & G & $1947-99-99$ & 003S070W & 28 & & G3 & s3 & $\mathrm{H}$ & - & & \\
\hline 8,878 & $\begin{array}{l}\text { Vascular } \\
\text { Plants }\end{array}$ & Carex saximontana & $\begin{array}{l}\text { Rocky Mountain } \\
\text { sedge }\end{array}$ & G & $1920-05-29$ & 004S070W & 35 & & G5 & $\mathrm{S} 1$ & $\mathrm{H}$ & - & & \\
\hline 4,986 & $\begin{array}{l}\text { Vascular } \\
\text { Plants }\end{array}$ & Carex torreyi & Torrey sedge & G & 1903-99-99 & 003S070W & 28 & & G4 & $\mathrm{S} 1$ & $\mathrm{H}$ & - & & \\
\hline \multirow[t]{3}{*}{7,430} & Vascular & Spiranthes diluvialis & Ute ladies' tresses & $\mathrm{s}$ & $2008-07-28$ & 003S069w & & & G2G3 & $\mathrm{S} 2$ & B & $\mathrm{LT}$ & & \\
\hline & Plants & & & & & 003S069w & & & & & & & & \\
\hline & & & & & & 003S069w & & & & & & & & \\
\hline
\end{tabular}


Locations and Status of Rare and/or Imperiled Species and Natural Communities known from or likely to occur within a two-mile radius of 327 acres at NREL's South Table Mountain facility in Jefferson County, CO

Report generated: 25 June 2010

Copyright $\odot$ 2010. Colorado State University. Colorado Natural Heritage Program. All Rights Reserved.

\begin{tabular}{|c|c|c|c|c|c|c|c|c|c|c|c|c|c|c|}
\hline EO_ID & major group & scientific name & common name & Prec & last obs & $\begin{array}{l}\text { Town/ } \\
\text { Range }\end{array}$ & $\mathrm{Sec}$ & TRS Note & grank & srank & $\begin{array}{l}\text { eo- } \\
\text { rank }\end{array}$ & $E S A$ & fed stat & st stat \\
\hline 1,310 & Insects & $\begin{array}{l}\text { Callophrys mossii } \\
\text { schryveri }\end{array}$ & Moss's Elfin & G & $1982-04-28$ & $0035071 \mathrm{~W}$ & 36 & & G4T3 & $\mathrm{S} 2 \mathrm{~S} 3$ & $\mathrm{H}$ & - & & \\
\hline 7 & Insects & Celastrina humulus & Hops Feeding Azure & M & $1982-07-03$ & 003S070W & 33 & & G2G3 & $\mathrm{s} 2$ & $\mathrm{H}$ & - & & \\
\hline 9,087 & Insects & Polites origenes & Cross-line Skipper & M & 1929-07-04 & 003S070W & 33 & & G5 & S3 & $\mathrm{H}$ & - & & \\
\hline 468 & $\begin{array}{l}\text { Vascular } \\
\text { Plants }\end{array}$ & Carex saximontana & $\begin{array}{l}\text { Rocky Mountain } \\
\text { sedge }\end{array}$ & G & $1878-06-20$ & $0035071 \mathrm{~W}$ & 36 & & G5 & $\mathrm{S} 1$ & $\mathrm{H}$ & - & & \\
\hline
\end{tabular}




\title{
Data Dictionary for Potential Conservation Area Transcription Reports from the Colorado Natural Heritage Program
}

This Data Dictionary defines terms used in Potential Conservation Area (PCA) Reports exported by the Colorado Natural Heritage Program (CNHP) from our Biodiversity Tracking and Conservation System (BIOTICS) database.

\begin{abstract}
Introduction to Potential Conservation Areas
In order to successfully protect populations or occurrences, it is necessary to delineate conservation areas. These potential conservation areas focus on capturing the ecological processes that are necessary to support the continued existence of a particular element of natural heritage significance. Potential conservation areas may include a single occurrence of a rare element or a suite of rare elements or significant features.

The goal of the process is to identify a land area that can provide the habitat and ecological processes upon which a particular element or suite of elements depends for their continued existence. The best available knowledge of each species' life history is used in conjunction with information about topographic, geomorphic, and hydrologic features, vegetative cover, as well as current and potential land uses. The proposed boundary does not automatically exclude all activity. It is hypothesized that some activities will cause degradation to the element or the process on which they depend, while others will not. Consideration of specific activities or land use changes proposed within or adjacent to the preliminary conservation planning boundary should be carefully considered and evaluated for their consequences to the element on which the conservation unit is based.
\end{abstract}

\section{Element Occurrence}

An Element Occurrence (EO) is defined as a specific example of an Element at a geographic location characterized by a habitat capable of sustaining or contributing to the survival of the species, or by a landscape that supports the ecological integrity of the community.

\section{Element}

A biodiversity unit of conservation attention and action for which a Heritage Conservation Status Rank is assigned.

Elements may be recognized at any taxonomic level (although typically are only recognized at the species level and below for organisms, and the Ecological System, Alliance, and Association levels for communities).

\section{Elements may also be recognized for biodiversity units for which there is no systematic hierarchy} (e.g., animal assemblages, community Complexes).

\begin{abstract}
Elements may be native or exotic at a particular location and collectively represent the full array of biological and ecological diversity for the geographic area covered. Elements may serve as the targets of Heritage inventory. Typically, these targets include native, regularly occurring vulnerable species (including infraspecific taxa and populations) and exemplary ecological communities.
\end{abstract}

\section{REPORT HEADER}

\section{Name}

The official CNHP site name, usually corresponding to a local place name or nearby geographic feature.

\section{Site Code}

Unique identifier previously used in the BCD for a site record. 


\title{
IDENTIFIERS
}

\section{Site ID}

Unique identifier for a site.

\section{Site Class}

Value that indicates whether a site is a Potential Conservation Area (PCA) or Network of Conservation Areas (NCA).

Domain values for Site Class are:

$$
\begin{aligned}
& \text { PCA } \\
& \text { NCA }
\end{aligned}
$$

\section{Site Alias}

Other names commonly associated with the PCA. These can include informal names, old site names, names used by other offices or cooperating organizations, or the original survey site name.

\section{Network of Conservation Areas (NCA)}

A Network of Conservation Areas (NCA) will fit one of the following definitions:

\begin{abstract}
A. A landscape area that encompasses Potential Conservation Areas (PCAs) that share similar species or natural communities and ecological processes. NCAs include unoccupied or unsurveyed areas that are within the same ecological system that the species or natural communities require. NCAs contain PCAs with an obvious repeating pattern (that is, the same species or natural communities are in each associated PCA).
\end{abstract}

B. A mostly intact, lightly fragmented landscape that supports wide-ranging species and large scale disturbances. NCAs include unoccupied or unsurveyed areas that demonstrate the connectivity of the landscape. NCAs contain PCAs that may occur at a variety of ecological scales.

\section{NCA Site ID}

Site ID of the NCA associated with this PCA.

\section{NCA Site Code}

Site code of the NCA associated with this PCA.

\section{NCA Site Name}

Official CNHP site name of the NCA associated with this PCA.

\section{Site Relations}

Comments that explain the relationship between this site and any nested, overlapping, or adjacent sites.

\section{LOCATORS}

\section{Nation \\ State}




\section{Latitude}

Degrees, Minutes, Seconds. Datum is NAD 27. Calculated in GIS.

\section{Longitude}

Degrees, Minutes, Seconds. Datum in NAD 27. Calculated in GIS.

USGS 7.5 Minute Quadrangle

Calculated in GIS.

Quad Code

Quad Name

\section{County}

Calculated in GIS.

\section{Watershed Code}

8 digit U.S.G.S. hydrological unit code. Calculated in GIS.

\section{Watershed Name}

U.S.G.S. watershed name. Calculated in GIS.

\section{Township/Range/Section (TRS) - Public Land Survey System}

Calculated in GIS.

\section{Township/Range}

Section

Meridian

TRS Note

Site Directions [provided with Level 1 data only]

Specific directions to the site provided by the designer or version author.

\section{SITE DESCRIPTION}

\section{Minimum Elevation}

Minimum elevation provided by the designer or version author.

\section{Maximum Elevation}

Maximum elevation provided by the designer or version author.

\section{Site Description}

General visual description (or word picture) of the principal physical and natural features on the site.

\section{Key Environmental Factors}

Description of the driving factors or key environmental variables that are known to exert a major influence on the biota at the site (e.g., seasonal flooding, wind, soil type). 


\section{Climate Description}

General comments concerning climate and weather patterns, wind patterns, seasonal and annual variations, as well as temperature and precipitation patterns characteristic of the site.

\section{Land Use History}

Comments concerning past land uses on this site (such as mining, logging, shifting cultivation, etc.).

\section{Cultural Features}

Comments concerning any historic, cultural, or archaeological features found on the site (e.g., pictographs, petroglyphs, burial mounds, prehistoric artifacts).

\section{SITE DESIGN}

Site Map

Indicates whether a site boundary was field verified or drawn from desktop references.

Domain values for Site Map are:

$\mathrm{P}$ - partial; drawn from desktop references

$\mathrm{Y}$ - field verified by CNHP personnel

\section{Mapped Date}

Date site boundary was last redrawn.

\section{Designer}

CNHP biologist responsible for drawing the site boundary.

\section{Boundary Justification}

Explanation of the biological rationale used to determine the ecological boundaries for the site.

\section{Primary Area}

Area of PCA polygon. Calculated in GIS.

\section{SITE SIGNIFICANCE}

\section{Biodiversity Significance Rank}

Value that indicates the rating that best describes the significance of the site in terms of its biological diversity.

Domain values for Biodiversity Significance are:

B1: Outstanding Biodiversity Significance

B2: Very high Biodiversity Significance

B3: High Biodiversity Significance

B4: Moderate Biodiversity Significance

B5: General interest/open space

B?: Unknown 


\section{Biodiversity Significance Comments}

Comments that justify the rating assigned for the site in the Biodiversity Significance field.

\section{Other Values Rank}

Value that indicates the rating that best describes the significance of the site in terms of its aesthetic, recreational, open space, and other ecological values; this includes its role in maintaining ecosystem health (e.g., by providing game and wildlife habitat, aquifer recharge functions, erosion control).

Domain values for Other Values are:

V1 - Outstanding values

V2 - High values

V3 - Moderate values

V4 - No known values

V5 - Negative or counter values

V? - Unknown

(null) - Not assessed

\section{Other Values Comments}

Comments that justify the rating assigned for the site in the Other Values field.

\section{Protection Urgency Rank [provided with Level 1 data only]}

Value that indicates the rating that best describes the urgency to protect the site. The urgency for protection action (not to be confused with the urgency for management action) will generally increase with impending threats to the site until legal, political, or other administrative measures are taken.

Domain values for Protection Urgency are:

P1 - Immediately threatened/outstanding opportunity

P2 - Threat/opportunity within 5 years

P3 - Definable threat/opportunity, but not within 5 years

P4 - No threat or special opportunity

P5 - No action to be taken on this site

P? - Unknown

Protection Urgency Comments [provided with Level 1 data only]

Comments that justify the rating assigned for the site in the Protection Urgency field.

\section{Management Urgency Rank [provided with Level 1 data only]}

Value that indicates the rating that best describes the urgency to manage one or more Elements at the site. The urgency for management action (not to be confused with the urgency for legal protection action) requires stewardship intervention in order to maintain EOs at the site. 
Domain values for Management Urgency are:

M1 - Essential within 1 year to prevent loss

M2 - Essential within 5 years to prevent loss

M3 - Needed within 5 years to maintain quality

M4 - Not needed now; no current threats; may need in future

M5 - Not needed; no threats anticipated

M? - Unknown

Management Urgency Comments [provided with Level 1 data only]

Comments that justify the rating assigned for the site in the Management Urgency field.

\section{LAND MANAGEMENT ISSUES}

\section{Land Use Comments}

Description of the current and past land use, improvements, and structures on the site.

\section{Natural Hazard Comments}

Description of the potential natural hazards (e.g., cliffs, caves, waterfalls) on the site, along with any precautions that should be taken by stewards.

\section{Exotics Comments}

Description of potentially damaging exotic (i.e., alien) flora and fauna (e.g., kudzu, honeysuckle, purple loosestrife, periwinkle, English ivy, feral goats, pigs) on the site.

\section{Offsite}

Description of off-site land uses (e.g., farming, logging, grazing, dumping, watershed diversion), and how these uses might affect the site, Elements on the site, and management of the site.

\section{Information Needs}

Summary of the information that is still needed in order to effectively manage the site and Elements on it.

\section{Management Needs [provided with Level 1 Data only]}

Summary of the expected management needs for the site and the Elements on it.

\section{Managed Area Relations [provided with Level 1 Data only]}

Explanation of the site/Managed Area relationship, if a Managed Area has been (or will be) established to protect the site.

\section{Protection Comments [provided with Level 1 Data only]}

Summary of the general level of protection currently afforded the site that indicates the current protection status of component Tracts. 


\title{
ASSOCIATED ELEMENTS OF BIODIVERSITY
}

(Tracked Elements known from the area of a given PCA.)

\begin{abstract}
Element
A biodiversity unit of conservation attention and action for which a Heritage Conservation Status

Rank is assigned.

Elements may be recognized at any taxonomic level (although typically are only recognized at the species level and below for organisms, and the Ecological System, Alliance, and Association levels for communities).

Elements may also be recognized for biodiversity units for which there is no systematic hierarchy (e.g., animal assemblages, community Complexes).

Elements may be native or exotic at a particular location and collectively represent the full array of biological and ecological diversity for the geographic area covered. Elements may serve as the targets of Heritage inventory. Typically, these targets include native, regularly occurring vulnerable species (including infraspecific taxa and populations) and exemplary ecological communities.
\end{abstract}

\section{Element State ID}

Unique state identifier for an Element.

\section{State Scientific Name}

State scientific name for an Element having occurrences associated with this PCA.

\section{State Common Name}

State common name for an Element having occurrences associated with this PCA.

\section{Global Rank}

The global element rank that best characterizes the relative rarity or endangerment of the element worldwide. Factors other than the number of occurrences may be considered when assigning a global rank. Global ranks are derived primarily by staff at the Central Heritage Conservation Science Department, unless CNHP has lead responsibility for that element.

Domain values for Global Rank are:

G1 - Globally critically imperiled; typically 5 or fewer occurrences

G2 - Globally imperiled; typically 6 to 20 occurrences

G3 - Globally vulnerable; typically 21 to 100 occurrences

G4 - Globally apparently secure; usually $>100$ occurrences

G5 - Globally demonstrably secure although it may be rare in parts of its range

$\mathrm{G} \# \mathrm{G} \#$ - A range between two of the numeric ranks; indicates uncertainty about the rarity of the element

G? - Unranked; element is not yet ranked globally

GU - Unrankable; not enough information is known

GH - Historically known with hopes of rediscovery

GX - Extinct; unlikely to be rediscovered

T\# - Rank applies to a subspecies or variety

$\mathrm{Q}$ - Taxonomic status is questionable 
$\mathrm{C}$ - Element is extant only in captivation or cultivation

GNR - Not ranked globally

\section{State Rank}

The state element rank that best characterizes the relative rarity or endangerment of the element statewide. Factors other than the number of occurrences may be considered when assigning a state rank. State ranks are derived by CNHP staff.

Domain values for State Rank are:

S1 - State critically imperiled; typically 5 or fewer occurrences

S2 - State imperiled; typically 6 to 20 occurrences

S3 - State vulnerable; typically 21 to 100 occurrences

S4 - State apparently secure; usually $>100$ occurrences

S5 - State demonstrably secure

S\#S\# - A range between two of the numeric ranks; indicates uncertainty about the rarity of the element

S? - Unranked; element is not yet ranked in the state

$\mathrm{SU}$ - Unrankable; not enough information is known

SH - Historically known with hopes of rediscovery

SX - Extinct; unlikely to be rediscovered

SE - An exotic established in the state; native to a nearby region

SA - Accidental; includes species (usually birds or butterflies) recorded once or twice or only at very great intervals, hundreds or thousands of miles outside their usual range

$\mathrm{B}$ - Rank refers to the breeding population of the element

$\mathrm{N}$ - Rank refers to the nonbreeding population of the element

$\mathrm{C}$ - Element is extant only in captivation or cultivation

SNR - Not ranked in the state

\section{Driving Site Rank}

Yes or No, indicates whether this EO is the EO which is driving the biodiversity rank of this PCA. A combination of Global Imperilment Rank, State Imperilment Rank, and EO Rank factors determine if a given EO drives the biodiversity rank of a PCA that supports it.

\section{REFERENCES}

\section{Reference ID}

The identifier for a reference available for this PCA.

\section{Full Citation}

Formal citation for a reference associated with the PCA.

\section{ADDITIONAL TOPICS}

\section{Additional Topics}

Specific comments on any significant additional nonstandard topics that have not been formally addressed by one of the standard fields in this record. 


\section{VERSION}

\section{Version Date}

Date report information for the PCA was last reviewed or updated.

\section{Version Author}

Author of the current version of the transcription in this report. 


\section{Potential Conservation Area (PCA) Report}

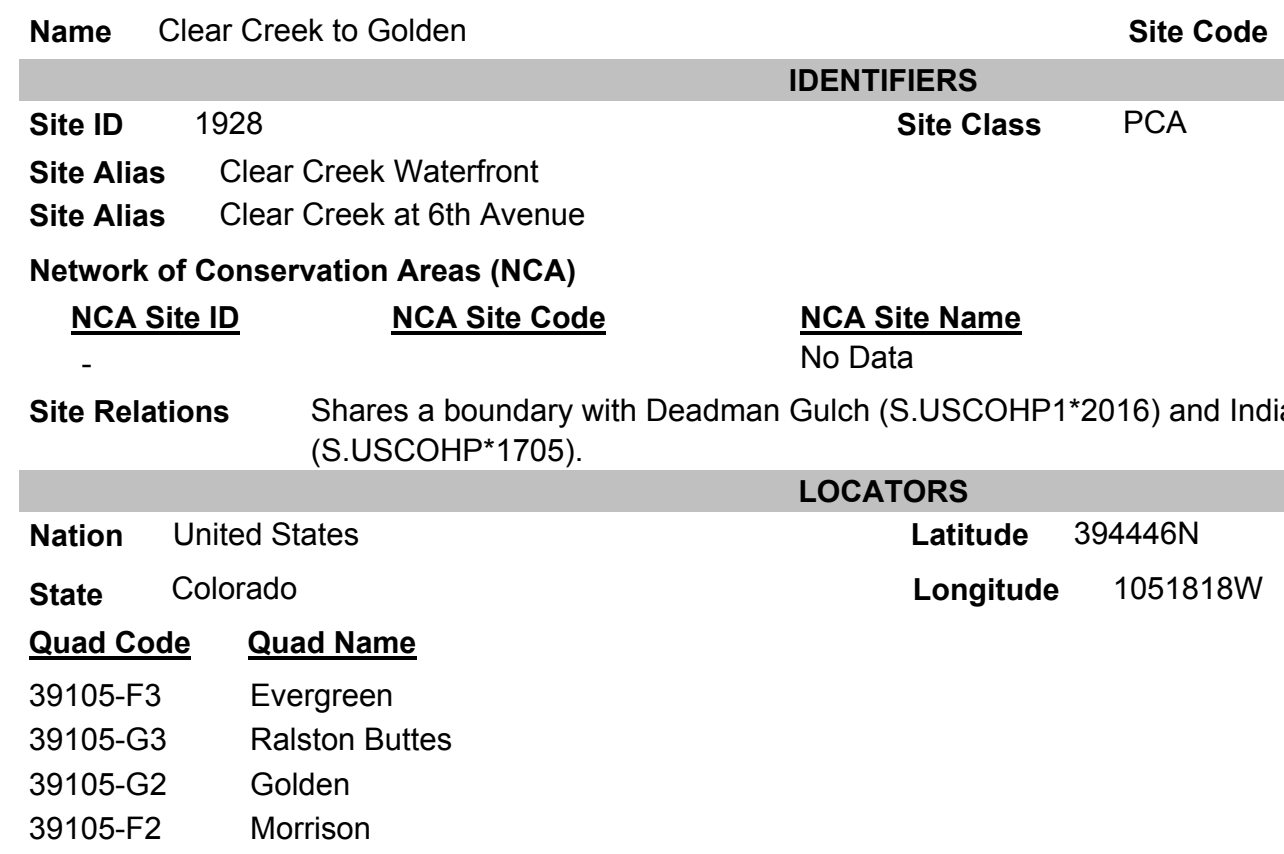

County

\begin{tabular}{|c|c|c|c|c|c|}
\hline Watershed Code & Waters & ed Name & & & \\
\hline 10190004 & Clear & & & & \\
\hline Township/Range & & Section & $\underline{\text { Meridian }}$ & $\underline{\text { Note }}$ & \\
\hline 003S071W & & 35 & $6 \mathrm{P}$ & & \\
\hline 003S071W & & 36 & $6 \mathrm{P}$ & & \\
\hline 004S071W & & 01 & $6 \mathrm{P}$ & & \\
\hline 004S070W & & 05 & $6 \mathrm{P}$ & & \\
\hline 003S070W & & 29 & $6 \mathrm{P}$ & & \\
\hline 003S070W & & 32 & $6 \mathrm{P}$ & & \\
\hline 003S070W & & 31 & $6 \mathrm{P}$ & & \\
\hline 004S070W & & 06 & $6 \mathrm{P}$ & & \\
\hline 003S070W & & 33 & $6 \mathrm{P}$ & & \\
\hline 003S070W & & 28 & $6 \mathrm{P}$ & & \\
\hline \multicolumn{6}{|c|}{ SITE DESCRIPTION } \\
\hline Minimum Elevation & & - & & - & Meters \\
\hline Maximum Elevation & & $-\quad F$ & & - & Meters \\
\hline
\end{tabular}

Site Description

The site includes a narrow band of riparian vegetation along Clear Creek and adjacent slopes in the canyon.

\section{Key Environmental Factors}

No Data

Climate Description

No Data

Land Use History

No Data

\section{Cultural Features}

No Data

$\begin{array}{llrr}\text { Site Map } & \text { P - Partial } & \text { SITE DESIGN } & \\ \text { Designer } & \text { Panjabi, S.S. } & \text { Mapped Date } & 09 / 11 / 2008 \\ & & & \\ & & & \\ \end{array}$




\section{Potential Conservation Area (PCA) Report}

Name Clear Creek to Golden

Site Code S.USCOHP1*1928

Boundary Justification

Includes the plant occurrences and significant up and downstream areas. Although most local ecological processes are included in the boundary, the largest scale forces that support the plants originate upstream; therefore, watershed management strategies that support the conservation of the elements will be necessary for long-term viability.

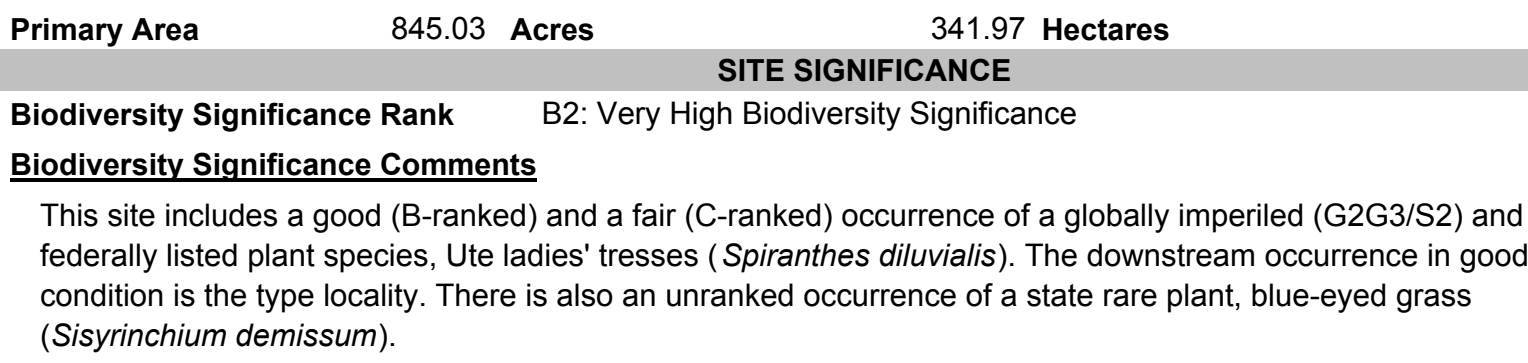

\section{Other Values Rank No Data}

Other Values Comments

No Data

LAND MANAGMENT ISSUES

\section{Land Use Comments No Data \\ Natural Hazard Comments No Data \\ Exotics Comments \\ No Data \\ Offsite \\ No Data \\ Information Needs \\ No Data}

\begin{tabular}{|c|c|c|c|c|c|}
\hline \multicolumn{6}{|c|}{ ASSOCIATED ELEMENTS OF BIODIVERSITY } \\
\hline $\begin{array}{l}\text { Element } \\
\text { State ID }\end{array}$ & $\underline{\text { State Scientific Name }}$ & $\underline{\text { State Common Name }}$ & $\begin{array}{l}\text { Global } \\
\text { Rank }\end{array}$ & $\begin{array}{l}\text { State } \\
\text { Rank } \\
\end{array}$ & $\begin{array}{l}\text { Driving } \\
\text { Site Rank }\end{array}$ \\
\hline 17998 & Spiranthes diluvialis & Ute ladies' tresses & G2G3 & $\mathrm{S} 2$ & No \\
\hline 24424 & Sisyrinchium demissum & blue-eyed grass & G5 & S2 & No \\
\hline 17998 & Spiranthes diluvialis & Ute ladies' tresses & G2G3 & $\mathrm{S} 2$ & Yes \\
\hline
\end{tabular}

\section{Reference ID}

\section{Full Citation}

No Data

-

ADDITIONAL TOPICS

\section{Additional Topics}

Original site design by Pague, C.A. 1994-09-12.

$\begin{array}{lll}\text { Version Date } & \text { 09/11/2008 } & \text { VERSION } \\ \text { Version Author } & \text { Panjabi, S.S. }\end{array}$




\section{Potential Conservation Area (PCA) Report}

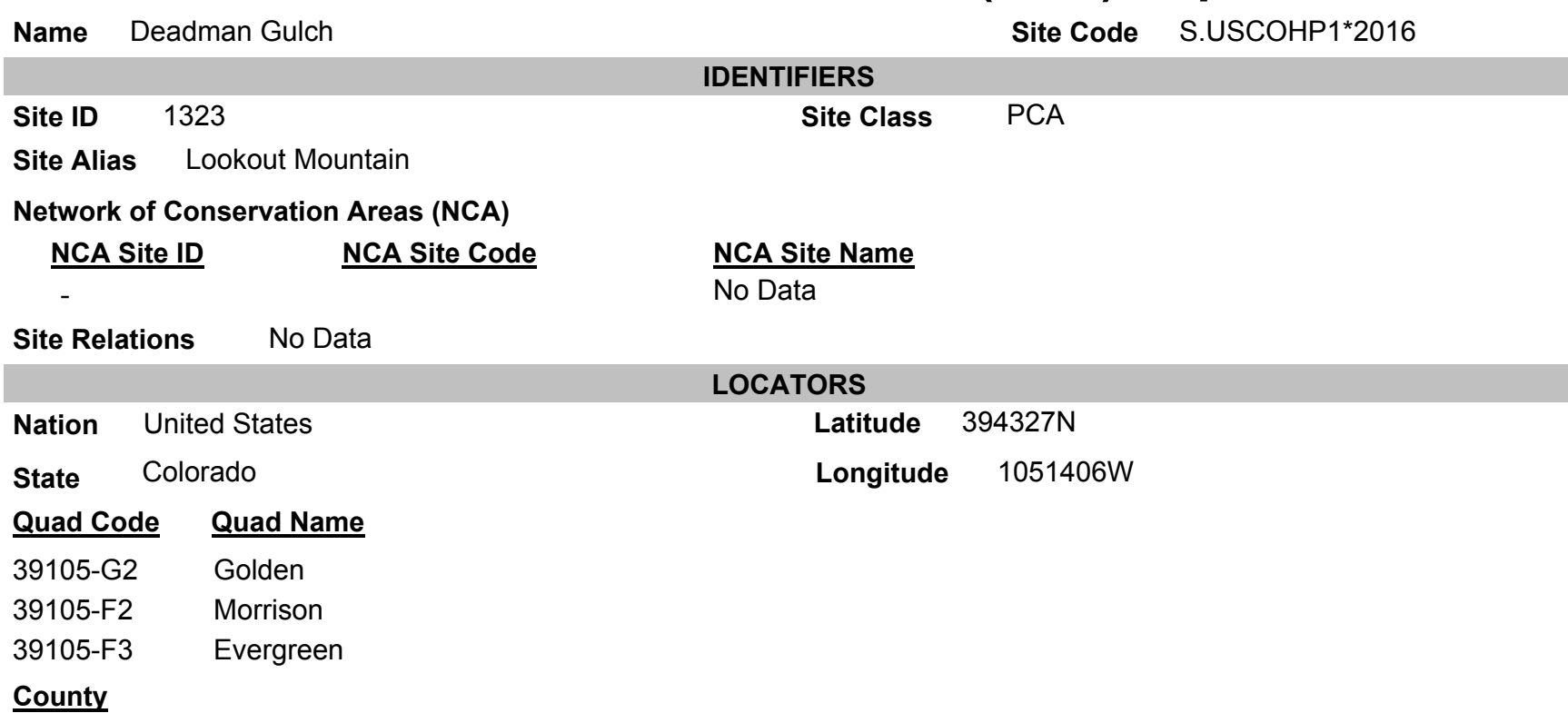

\section{Watershed Code \\ 10190004 \\ 10190002}

Township/Range

004S070W

004S070W

004S070W

004S070W

004S070W

003S070W

004S070W

004S070W

004S070W

004S070W

003S070W

004S070W

004S070W

003S070W

\section{Watershed Name \\ Clear \\ Upper South Platte}

$\begin{array}{lrlrl} & & & \text { SITE DESCRIPTION } & \\ \text { Minimum Elevation } & 5,800.00 & \text { Feet } & 1,768.00 & \text { Meters } \\ \text { Maximum Elevation } & 7,220.00 & \text { Feet } & 2,201.00 & \text { Meters }\end{array}$

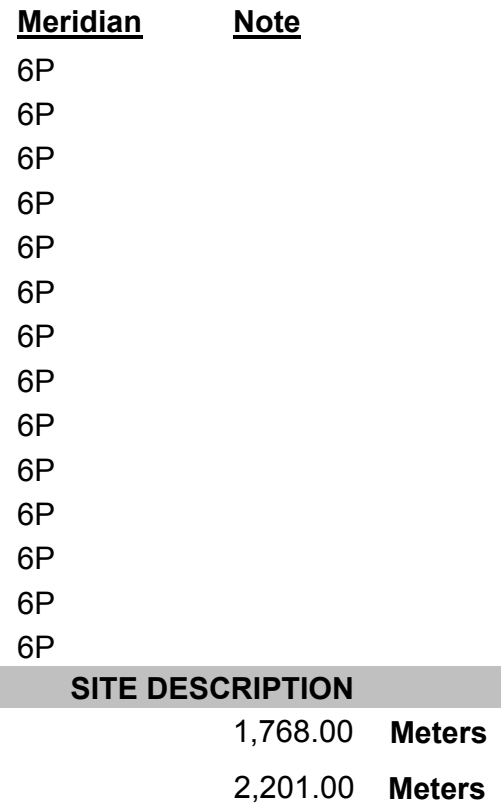

\section{Site Description}

[Pineda 1998:] Elevation: 5800 to 7220 feet (1767 to $2200 \mathrm{~m}$ ). The geology of this area is mostly of the Ratake-Cathedral-Rock outcrop complex containing slopes and ridges that face east, west or south, with 25 to 60 percent slopes. The soils in this complex are mostly formed in clayey and loamy material derived from sedimentary rocks. Soils are suitable for wildlife habitat, woodland, recreation areas, pasture, grazing, community development, and some crops. Soils are typcially shallow and well drained. Soil blowing is minimal, and rock fragments make up about 35 to 80 percent of the soil volume. A mosaic of plant communities exists here. The higher areas are mostly Ponderosa pine communities with a graminoid understory, most of which is young growth, and no fire scars are apparent. Midslopes are dominated mostly by mountain mahogany shrubland communities and yucca with big blue stem grass in the understory. Bottoms of slopes are typically of shrubland/grassland communities, mostly plum, chokecherry, sumac andbuckbrush, with an abundant accumulation of exotic flora at this level. Hillsides are definitely much less weedy than upslope. Graminoids at all levels include one or more of the following: big blue stem, little blue stem, blue grama, western weat, and buffalo grass. 


\section{Potential Conservation Area (PCA) Report}

Name Deadman Gulch

Site Code S.USCOHP1*2016

\section{Key Environmental Factors}

Fire, erosion, low annual precipitation.

\section{Climate Description}

Average annual precipitation is 17 to 20 inches $(43$ to $50 \mathrm{~cm}$ ). Average annual air temperature is 43 to 47 degrees Fahrenheit (6.1 to $8.3 \mathrm{C}$ ). Average frost-free season is 76 to 125 days, depending on elevation.

\section{Land Use History}

At least within the last 100 years, this area was used for grazaing of livestock.

\section{Cultural Features}

No Data

$\begin{array}{llrr}\text { Site Map } & \text { P - Partial } & \text { SITE DESIGN } & \\ \text { Designer } & \text { Pineda, P.M. } & \text { Mapped Date } & 01 / 11 / 1999\end{array}$

\section{Boundary Justification}

The boundaries are meant to enclose a small watershed, and management should consider what upslope management has on the lower slopes. It is meant to allow for genetic exchange to occur between rare element occurrences. Areas adjacent or encompassing residential development are included to manage for weedy floral invasions.

$\begin{array}{lrl}\text { Primary Area } & 4,865.77 \text { Acres } & 1,969.12 \text { Hectares }\end{array}$

\section{Biodiversity Significance Rank B3: High Biodiversity Significance \\ Biodiversity Significance Comments}

This site contains occurrences of a G2 butterfly, as well as a diverse variety of butterfly and skipper occurrences that are peculiar to mixed grass communities of the Colorado Front Range.

\section{Other Values Rank No Data}

Other Values Comments No Data

\section{LAND MANAGMENT ISSUES}

\section{Land Use Comments}

Historically probably used for grazing of livestock, and more recently has been found to be suitable for residential development.

\section{Natural Hazard Comments} No Data

\section{Exotics Comments}

Bromus tectorum, Bromus japonicus, Alyssum, Linaria dalmatica, and very thick, impenetrable stands of Carduus nutans, especially at the bottom of the slopes.

\section{Offsite}

Housing developments, roads, freeways, highways, trails, retail development, water diversions, City of Denver, City of Golden, quarries, agricultural develompent, livestock grazing.

\section{Information Needs} No Data

\begin{tabular}{|c|c|c|c|c|c|}
\hline \multicolumn{6}{|c|}{ ASSOCIATED ELEMENTS OF BIODIVERSITY } \\
\hline Element & & & Global & State & Driving \\
\hline State ID & $\underline{\text { State Scientific Name }}$ & $\underline{\text { State Common Name }}$ & $\underline{\text { Rank }}$ & $\underline{\text { Rank }}$ & Site Rank \\
\hline 17076 & Grammia sp. 1 & A Tiger Moth & G2G3 & SNR & No \\
\hline 16895 & Atrytone arogos & Arogos Skipper & G3 & S2 & No \\
\hline 16895 & Atrytone arogos & Arogos Skipper & G3 & S2 & No \\
\hline 20146 & Celastrina humulus & Hops Feeding Azure & G2G3 & S2 & Yes \\
\hline 22043 & Doa ampla & A Moth & GNR & S1 & No \\
\hline 19678 & Erynnis martialis & Mottled Dusky Wing & G3 & S2S3 & No \\
\hline
\end{tabular}




\section{Potential Conservation Area (PCA) Report}

Name Deadman Gulch

Site Code S.USCOHP1*2016

\begin{tabular}{lll}
$\frac{\text { Reference ID }}{173854}$ & $\frac{\text { Full Citation }}{\text { Field Survey (temporary placeholder citation) }}$ \\
Additional Topics & & REFERENCES \\
\hline No Data & VERSION \\
Version Date & $01 / 11 / 1999$ & \\
Version Author & Pineda, P.M. &
\end{tabular}




\section{Potential Conservation Area (PCA) Report}

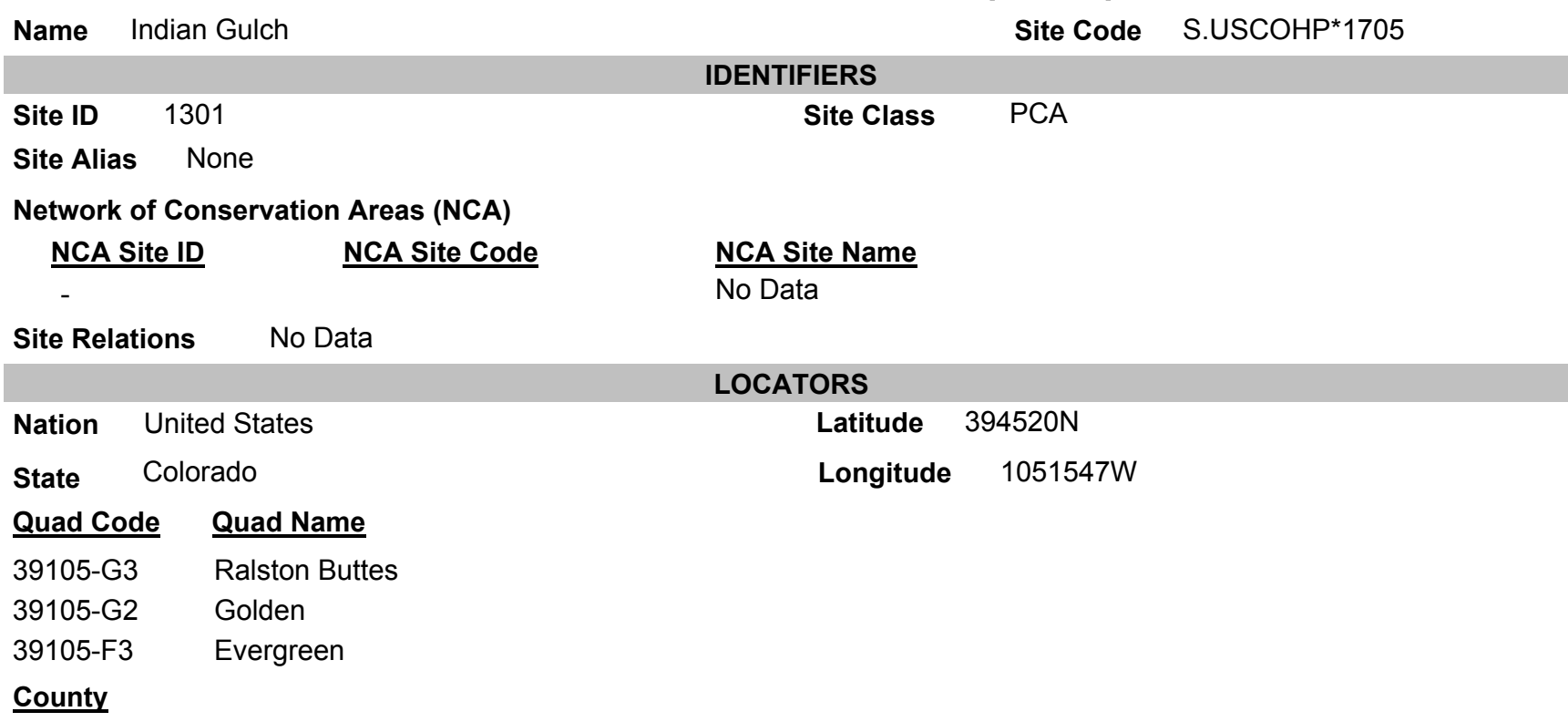

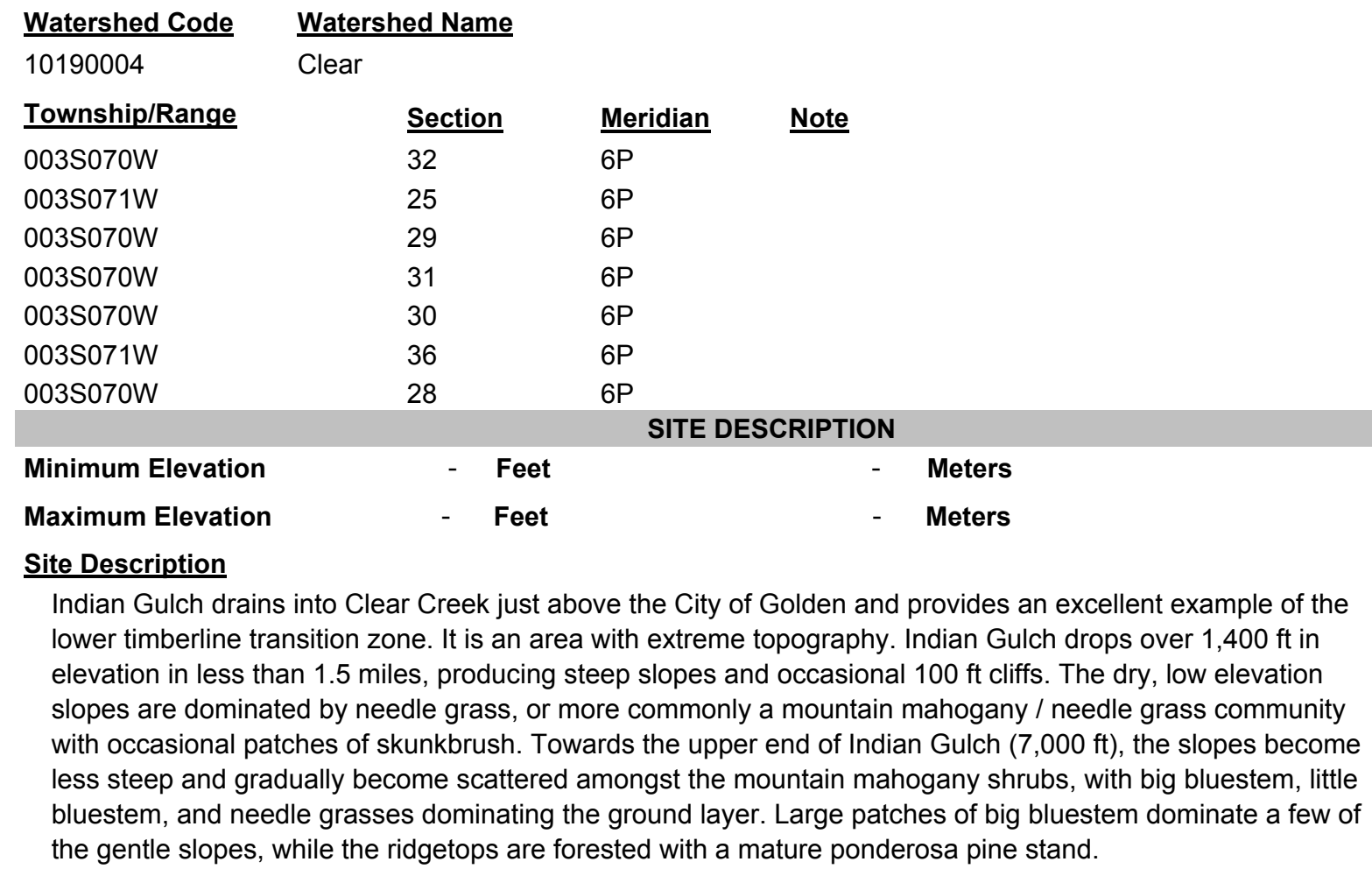

Key Environmental Factors

No Data

Climate Description

No Data

Land Use History

No Data

Cultural Features

No Data

$\begin{array}{llrr}\text { Site Map } & \text { P-Partial } & \text { SITE DESIGN } & \\ \text { Designer } & \text { Pague, C.A. } & \text { Mapped Date } & 09 / 12 / 1994 \\ & & & \\ & & & \\ \end{array}$

Copyright $\odot$ 2010. Colorado State University. Colorado Natural Heritage Program. All Rights Reserved. 


\section{Potential Conservation Area (PCA) Report}

Name Indian Gulch

Site Code S.USCOHP*1705

\section{Boundary Justification}

Includes all the significant plant associations and rare plants plus the mature ponderosa pine forest. The watershed boundaries and slightly beyond are used as the best approximation of a means of assuring the protection of the integrity of the ecosystem. Zoological inventory was not conducted at this site, but is highly recommended.

Primary Area $1,640.05$ Acres

663.71 Hectares

\section{SITE SIGNIFICANCE}

\section{Biodiversity Significance Rank B2: Very High Biodiversity Significance}

\section{Biodiversity Significance Comments}

Site hosts good to fair (B-ranked, BC-ranked) occurrences of globally imperiled (G2/S2) natural community types (Pinus ponderosa / Cercocarpus montanus / Andropogon gerardii and Cercocarpus montanus / Hesperostipa comata) as well as a poor (D-ranked) occurrence of a globally imperiled (G2/S2), federally Threatened plant species, Ute ladies' tresses (Spiranthes diluvialis).

\section{Other Values Rank No Data}

Other Values Comments

No Data

\section{LAND MANAGMENT ISSUES}

\section{Land Use Comments} No Data

\section{Natural Hazard Comments No Data}

\section{Exotics Comments}

No Data

\section{Offsite} No Data

\section{Information Needs}

No Data

\begin{tabular}{|c|c|c|c|c|c|}
\hline \multicolumn{6}{|c|}{ ASSOCIATED ELEMENTS OF BIODIVERSITY } \\
\hline$\frac{\text { Element }}{\text { State ID }}$ & State Scientific Name & State Common Name & $\begin{array}{l}\text { Global } \\
\text { Rank }\end{array}$ & $\begin{array}{l}\text { State } \\
\text { Rank }\end{array}$ & $\begin{array}{l}\text { Driving } \\
\text { Site Rank }\end{array}$ \\
\hline 24587 & $\begin{array}{l}\text { Pinus ponderosa / Cercocarpus montanus / } \\
\text { Andropogon gerardii Wooded Herbaceous } \\
\text { Vegetation }\end{array}$ & $\begin{array}{l}\text { Foothills Ponderosa Pine Scrub } \\
\text { Woodlands }\end{array}$ & G2 & $\mathrm{S} 2 ?$ & No \\
\hline 17998 & Spiranthes diluvialis & Ute ladies' tresses & G2G3 & S2 & No \\
\hline 24531 & $\begin{array}{l}\text { Cercocarpus montanus / Hesperostipa comata } \\
\text { Shrubland }\end{array}$ & Mixed Foothill Shrublands & G2 & $\mathrm{S} 2$ & Yes \\
\hline \multicolumn{6}{|c|}{ REFERENCES } \\
\hline \multicolumn{2}{|c|}{ Reference ID } & & & & \\
\hline- & No Data & & & & \\
\hline
\end{tabular}

\section{Additional Topics}

ADDITIONAL TOPICS

No Data

\section{VERSION}

Version Date $\quad$ 09/12/1994

Version Author Pague, C.A. 


\section{Potential Conservation Area (PCA) Report}

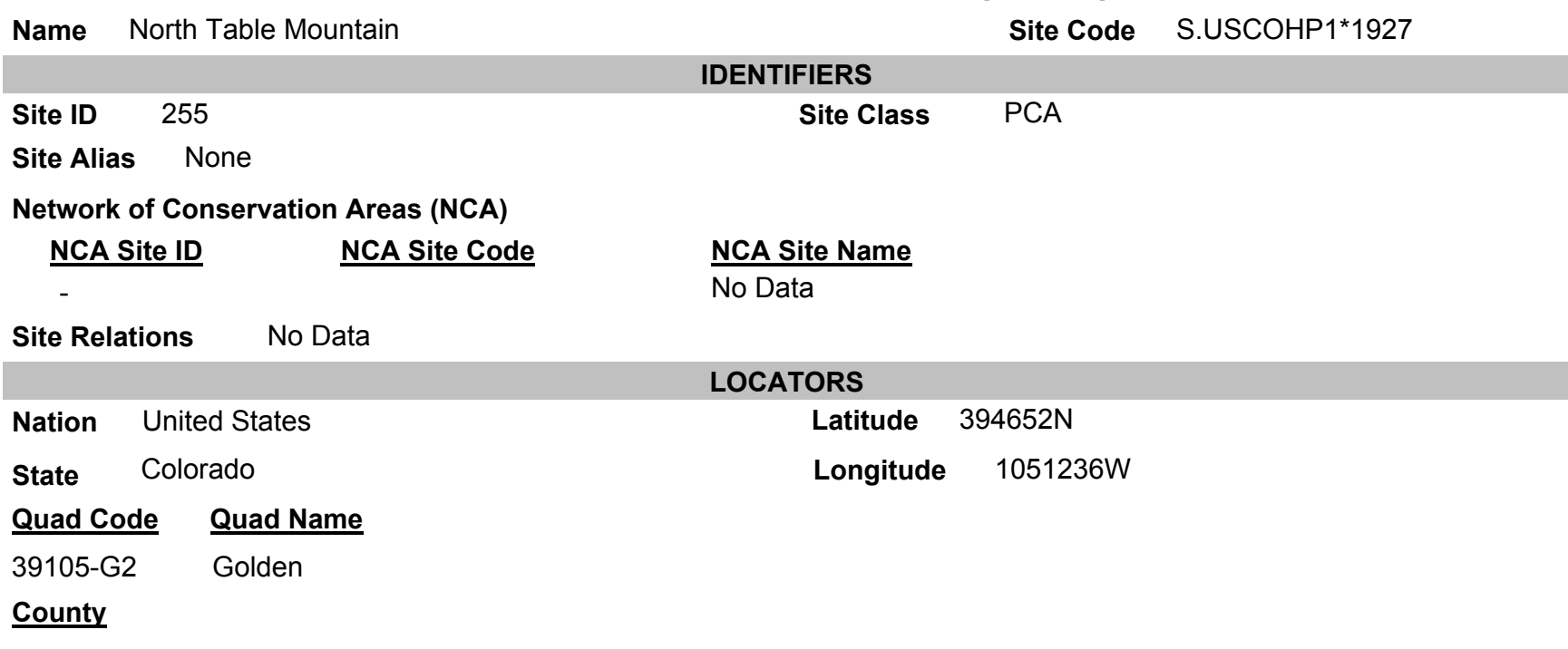

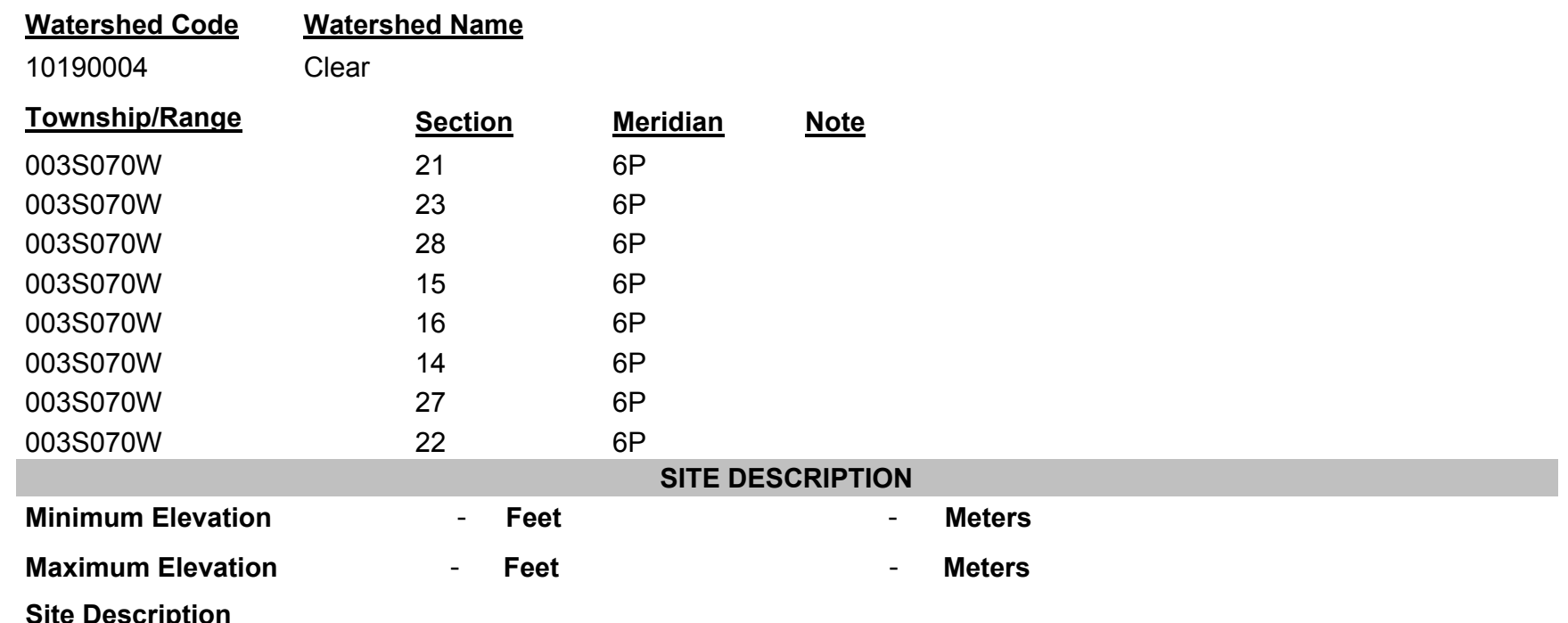

The North Table Mountain is a prominent landmark in the Denver-Golden area and has little development on the slopes or on top of the mesa. The mountain is a mesa that is surrounded by the Great Plains except for it's counter part, South Mountain. North Table Mountain consists of rolling hills, extensive grasslands, shrublands, riparian areas, rock outcrops, and small ponds. The top of the mountain is primarily grassland with several types of grass. The slopes are densely vegetated with shrubs on the upper slopes and grasses on the lower slopes. There are three ponds on the top, one of which is naturally formed. Cattle have compacted the soil around all ponds, although tiger salamanders continue to breed in the natural ponds. The current use is light grazing, although certain areas show intensive past grazing. Mineral extraction has affected a number of sites, including a noticeable area on the southwest corner of the mesa. Prairie Falcons feed and probably breed on the cliffs of the mountain, although the nest could not be located. Two nests of Red-tailed Hawks were observed, only one active. Other raptures use the area during migration. It is worthy to note that a large number of ground-nesting birds use this site, including some short-grass prairie species such as the Lark Buntings. The butterfly community contained no rare species; rather, it showed the effects of disturbance. Kilburn and White (1992) give extensive coverage to this area and should be consulted for additional information.

\section{Key Environmental Factors}

No Data

Climate Description No Data

\section{Land Use History}

Historically the area has been used for homesteading, grazing, and mining. 


\section{Potential Conservation Area (PCA) Report}

Name North Table Mountain

\section{Cultural Features}

No Data
Site Code S.USCOHP1*1927

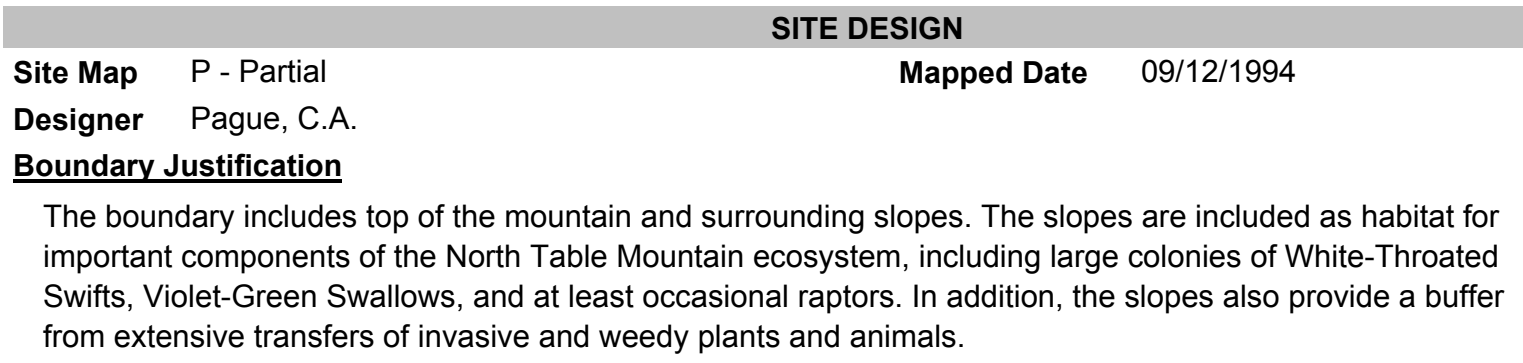
Primary Area
1,799.11 Acres
728.08 Hectares

\section{Biodiversity Significance Rank B3: High Biodiversity Significance \\ Biodiversity Significance Comments \\ The site supports a fair (C-ranked) occurrence of a globally imperiled (G1G2/S1S2) Hesperostipa comata Great Plains mixed grass prairie. Current field surveys could elevate the biodiversity rank.}

\section{Other Values Rank No Data \\ Other Values Comments No Data}

\section{LAND MANAGMENT ISSUES}

\section{Land Use Comments No Data \\ Natural Hazard Comments \\ No Data \\ Exotics Comments \\ No Data

$\frac{\text { Offsite }}{\text { No Data }}$
$\frac{\text { Information Needs }}{\text { No Data }}$

ASSOCIATED ELEMENTS OF BIODIVERSITY

\begin{tabular}{|c|c|c|c|c|c|}
\hline$\frac{\text { Element }}{\text { State ID }}$ & State Scientific Name & State Common Name & $\begin{array}{l}\text { Global } \\
\text { Rank }\end{array}$ & $\begin{array}{l}\text { State } \\
\text { Rank }\end{array}$ & $\begin{array}{l}\text { Driving } \\
\text { Site Rank }\end{array}$ \\
\hline 24703 & $\begin{array}{l}\text { Hesperostipa comata Colorado Front Range } \\
\text { Herbaceous Vegetation }\end{array}$ & Great Plains Mixed Grass Prairie & G1G2 & S1S2 & Yes \\
\hline
\end{tabular}

\begin{tabular}{|c|c|}
\hline & REFERENCES \\
\hline Reference ID & Full Citation \\
\hline 158822 & $\begin{array}{l}\text { Kilburn, P. and S. L. White. 1992. North Table Mountain its history and natural } \\
\text { features. Jefferson County Natural Association. }\end{array}$ \\
\hline
\end{tabular}

Additional Topics
No Data

$\begin{array}{lll} & & \text { VERSION } \\ \text { Version Date } & \text { 09/12/1994 } \\ \text { Version Author } & \text { Pague, C.A. }\end{array}$




\section{Potential Conservation Area (PCA) Report}

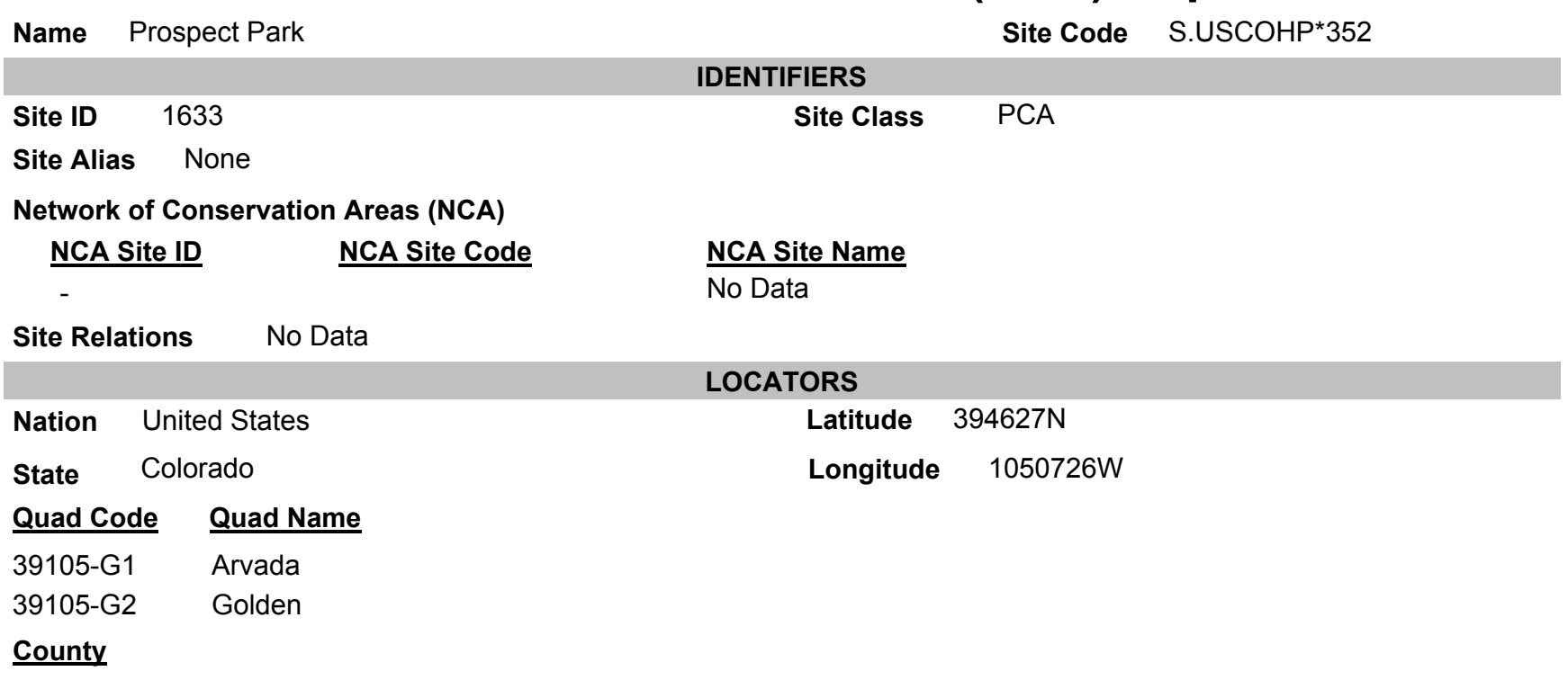

\section{Watershed Code \\ Watershed Name \\ 10190004 \\ Clear}

\begin{tabular}{|c|c|c|c|}
\hline Township/Range & Section & Meridian & $\underline{\text { Note }}$ \\
\hline 003S069W & 20 & $6 \mathrm{P}$ & \\
\hline 003S069W & 22 & $6 \mathrm{P}$ & \\
\hline 003S069W & 21 & $6 \mathrm{P}$ & \\
\hline 003S069W & 29 & $6 \mathrm{P}$ & \\
\hline 003S069W & 14 & $6 \mathrm{P}$ & \\
\hline 003S070W & 25 & $6 \mathrm{P}$ & \\
\hline $0035069 \mathrm{~W}$ & 19 & $6 \mathrm{P}$ & \\
\hline 003S069W & 30 & $6 \mathrm{P}$ & \\
\hline 003S069W & 28 & $6 \mathrm{P}$ & \\
\hline 003S069W & 26 & $6 \mathrm{P}$ & \\
\hline 003S069W & 23 & $6 \mathrm{P}$ & \\
\hline 003S070W & 24 & $6 \mathrm{P}$ & \\
\hline \multirow[t]{2}{*}{ 003S069W } & 27 & $6 \mathrm{P}$ & \\
\hline & & \multicolumn{2}{|c|}{ SITE DESCRIPTION } \\
\hline Minimum Elevation & $5,280.00$ Feet & & $1,609.00$ \\
\hline Maximum Elevation & $5,450.00 \quad$ Feet & & $1,661.00$ \\
\hline
\end{tabular}

\section{Site Description}

Clear Creek flows through the the Prospect Park site from west to east. The site includes the Wheat Ridge

Greenbelt, and a narrow area of connectivity to the Jefferson County Open Space to the west, which is evidently used by wildlife such as mountain lions. The overpass at Kipling Street fragments the site, and marks the eastern limit of the wide part of the greenbelt where residential development has not proliferated on the floodplain. From Kipling to the 44th Street overpass, the floodplain is more developed but significant areas of the riparian corridor and floodplain remain, with good examples of cottonwood riparian woodlands and marginal habitat for Ute ladies'-tresses (Spiranthes diluvialis). Most of the area was floodplain historically. The dominant vegetation types are cottonwood riparian woodlands and non-native grasslands dominated by smooth brome (Bromus inermis). There are many former creek channels which are typically dominated by wetland vegetation such as cattails (Typha spp.) and coyote willow (Salix exigua). Small creeks flow across the floodplain throughout the summer that are fed by seeps along the ridge to the south and by storm water runoff. Many of these creeks flow through dense vegetation that is virtually impenetrable, such as thickets of common buckthorn (Rhamnus cathartica). One occurrence of Ute ladies'-tresses is found within the site and is composed of approximately 18 subpopulations. Counts of over 400 individuals have been obtained in past years (i.e. 1999, a very good year at this location) for the two large subpopulations. A species of earth star (Mycenastrum sp. nov.) that is new to science has also been recently documented in the greenbelt west of 


\title{
Potential Conservation Area (PCA) Report
}

\author{
Name Prospect Park \\ Site Code S.USCOHP*352
}

the Miller Trailhead, and thus far is known only from this location. This report has yet to be verified, and the species description has not yet been published. Two areas in the site have components of rare communities. These include the Plains cottonwood - chokecherry woodland and the Plains cottonwood - snowberry woodland. The size of these areas is small and the condition is affected by the presence of non-native and invasive species. However, these examples may be remnants of the community type that naturally occurred there. Although their quality is poor, these areas could serve as references for restoration of these community types in other parts of the greenbelt where conditions would be appropriate for their establishment.

\section{Key Environmental Factors} No Data

\section{Climate Description}

No Data

\section{Land Use History}

Grazing, haying, homesteading, gravel mining, and recreational open space.

\section{Cultural Features}

No Data

\begin{tabular}{|c|c|c|c|}
\hline \multicolumn{4}{|c|}{ SITE DESIGN } \\
\hline Site Map & Y - Yes & Mapped Date & $01 / 17 / 2001$ \\
\hline Designer & Anderson, D.G. & & \\
\hline Boundary & ustification & & \\
\hline
\end{tabular}

The boundary encompasses a broad area known to support Spiranthes, inlcuding areas that the species is apparently expanding its range into appropriate habitat. Due to the occasional presence of potential habitat along the riparian corridor of Clear Creek, and the presence of several small subpopulations, all of the corridor east to a historically known location for the state-rare Ribes americana is included. The extensive cottonwood riparian woodland in the floodplain is circumscribed due to the potential for restoration to high quality natural communities throughout the area. The area west of the Miller Trailhead is included due to the presence of an undescribed species of earth star (Mycenastrum sp. nov.). In general, the entire floodplain, both private and public portions, between Youngsfield Street and 44th Street is included to encompass a functional portion of the riparian system on which the plants depend.

\section{Primary Area 3,860.84 Acres \\ 1,562.43 Hectares \\ SITE SIGNIFICANCE}

\section{Biodiversity Significance Rank B2: Very High Biodiversity Significance}

\section{Biodiversity Significance Comments}

This site is drawn for the Ute ladies'-tresses (Spiranthes diluvialis), a federally listed plant species. This site contains a good (B-ranked) occurence of this globally imperiled (G2G3/S2) species.

\section{Other Values Rank No Data}

Other Values Comments

No Data

\section{LAND MANAGMENT ISSUES}

\section{Land Use Comments}

No Data

\section{Natural Hazard Comments} No Data

\section{Exotics Comments}

Exotic species that are threatening Ute ladies'-tresses ( Spiranthes diluvialis) in order of priority are teasel (Dipsacus laciniatus), leafy spurge (Euphorbia esula), Canada thistle (Breea arvensis), Russian olive (Elaeagnus angustifolia), and yellow toadflax (Linaria vulgaris).

\section{Offsite}

No Data

Information Needs No Data 


\section{Potential Conservation Area (PCA) Report}

\begin{tabular}{|c|c|}
\hline Name & Prospect Park \\
\hline \multicolumn{2}{|l|}{ Element } \\
\hline$\overline{\text { State ID }}$ & State Scientific Name \\
\hline 17998 & Spiranthes diluvialis \\
\hline
\end{tabular}

\section{Full Citation}

Anderson, D. and J. Stevens. 2001. Wheat Ridge Open Space Biological Inventory. Prepared for the City of Wheat Ridge.

Site Code S.USCOHP*352

State Common Name

\begin{tabular}{|c|c|}
\hline Global & State \\
\hline Rank & $\underline{\text { Rank }}$ \\
\hline G2G3 & S2 \\
\hline
\end{tabular}

\section{ADDITIONAL TOPICS}

\section{Additional Topics \\ No Data}

$\begin{array}{lll} & & \text { VERSION } \\ \text { Version Date } & 01 / 17 / 2001 & \\ \text { Version Author } & \text { Anderson, D.G. }\end{array}$

